\title{
Metal-insulator transition in a weakly interacting many-electron system with localized single-particle states
}

\author{
D. M. Basko ${ }^{\text {a }}$, I. L. Aleiner ${ }^{\mathrm{b}}$, and B. L. Altshuler ${ }^{\mathrm{a}, \mathrm{b}, \mathrm{c}}$ \\ ${ }^{a}$ Department of Physics, Princeton University, Princeton, NJ 08544, USA \\ ${ }^{\mathrm{b}}$ Physics Department, Columbia University, New York, NY 10027, USA \\ ${ }^{\mathrm{c}}$ NEC-Laboratories America, 4 Independence Way, Princeton, NJ 085540, USA
}

\begin{abstract}
We consider low-temperature behavior of weakly interacting electrons in disordered conductors in the regime when all single-particle eigenstates are localized by the quenched disorder. We prove that in the absence of coupling of the electrons to any external bath dc electrical conductivity exactly vanishes as long as the temperatute $T$ does not exceed some finite value $T_{c}$. At the same time, it can be also proven that at high enough $T$ the conductivity is finite. These two statements imply that the system undergoes a finite temperature Metal to Insulator transition, which can be viewed as Anderson-like localization of many-body wave functions in the Fock space. Metallic and insulating states are not different from each other by any spatial or discrete symmetries.

We formulate the effective Hamiltonian description of the system at low energies (of the order of the level spacing in the single-particle localization volume). In the metallic phase quantum Boltzmann equation is valid, allowing to find the kinetic coefficients. In the insulating phase, $T<T_{c}$, we use Feynmann diagram technique to determine the probability distribution function for quantum-mechanical transition rates. The probability of an escape rate from a given quantum state to be finite turns out to vanish in every order of the perturbation theory in electron-electron interaction. Thus, electron-electron interaction alone is unable to cause the relaxation and establish the thermal equilibrium.
\end{abstract}

As soon as some weak coupling to a bath is turned on, conductivity becomes finite even in the insulating phase. Moreover, in the vicinity of the transition temperature it is much larger than phonon-induced hopping conductivity of non-interacting electrons. The reason for this enhancement is that the stability of the insulating state is gradually decreasing as the transition point is approached. As a result, a single phonon can cause a whole cascade of electronic hops.

Key words: Metal-insulator transition, Anderson localization, Fock space PACS: 73.20.Fz,, 73.23.-b,, 71.30.+h

Preprint submitted to Annals of Physics $\quad 2$ February 2008 


\section{Contents}

1 Introduction 3

2 Qualitative discussion $\quad 6$

2.1 Macroscopic manifestations of the many-body localization transition 6

2.2 Microscopic mechanism of the many-body localization transition $\quad 14$

3 Choice of the model 21

$\begin{array}{llr}4 & \text { Formalism. } & 25\end{array}$

4.1 Time evolution equations and basic definitions. 25

$\begin{array}{lll}4.2 & \text { Formulation of the problem. } & 31\end{array}$

4.3 Self-Consistent Born Approximations (SCBA and ImSCBA). 33

$5 \quad$ Stability and properties of the metallic phase. 38

$\begin{array}{lll}5.1 & \text { Condition for stability } & 38\end{array}$

5.2 Kinetic equation and transport coefficients. $\quad 42$

$6 \quad$ Stability of the insulating phase. 49

$\begin{array}{lll}6.1 & \text { Averaging procedure } & 50\end{array}$

6.2 Linearized ImSCBA equations $\quad 51$

6.3 Mayer-Mayer cluster expansion for the characteristic functions $\quad 53$

6.4 Statistical analysis for zero-dimensional system. $\quad 55$

6.5 Statistical analysis and metal-insulator transition in finitedimensional systems. $\quad 63$

$\begin{array}{lll}7 & \text { Validity of ImSCBA scheme } & 67\end{array}$

$\begin{array}{lll}7.1 & \text { Effect of the interaction renormalization. } & 68\end{array}$

$\begin{array}{lll}7.2 & \text { Effect of the particle permutations in the final state. } & 70\end{array}$

$\begin{array}{lll}\text { 7.3 Effect of the single-particle spectrum renormalization. } & 76\end{array}$

Email address: dbasko@princeton.edu (D. M. Basko). 
8 Conclusions $\quad 86$

9 Acknowledgements $\quad 88$

$\begin{array}{lll}\text { A Slow energy relaxation? } & 89\end{array}$

$\begin{array}{ll}\text { B Probability distributions for } \mathcal{B}_{\sigma, \kappa} . & 90\end{array}$

$\begin{array}{lll}\text { C } & \text { Cancellation of the cutoff } \Delta & 92\end{array}$

$\begin{array}{ll}\text { D Derivation of Eq. (151) } & 95\end{array}$

$\begin{array}{ll}\text { References } & 97\end{array}$

\section{Introduction}

Transport properties of conducting materials at low temperature $T$ are determined by an interplay between the interaction of the itinerant electrons with each other and the quenched disorder which creates a random potential acting on these electrons. In the absence of the electron-electron interaction all physics is dominated by the phenomenon of Anderson localization [1] - e.g., dc electrical conductivity $\sigma$ can be qualitatively different depending on whether one-particle wave functions of the electrons are localized or not. In the latter case $\sigma(T)$ has a finite zero-temperature limit, while in the former case $\sigma(T)$ vanishes when $T \rightarrow 0$. Therefore, Anderson localization of electronic states leads to the Metal to Insulator Transition at zero temperature.

When speaking about zero temperature, we need to consider only localization of the electronic states close to their Fermi level. The conductivity becomes finite at any finite temperature provided that extended states exist somewhere above the Fermi level. It is commonly accepted now that localized and extended states in a random potential can not be mixed in the one-electron spectrum and thus this spectrum in a very general case is a combination of bands of extended states and bands of localized states. A border between a localized and an extended band is called mobility edge. If the Fermi level is located inside a localized band and inelastic scattering of the electrons are completely absent, the conductivity should follow Arrhenius law $\sigma(T) \propto \exp \left(-E_{c} / T\right)$, where $E_{c}$ is the distance from the Fermi level to the closest mobility edge. Another common belief following from the scaling theory of Anderson localization [2,3], is that in low dimensionality $d$, namely at $d=1,2$ all states are localized in an arbitraryly small disorder, while for free electrons (no periodic potential) $E_{c}>0$ is finite at $d=3$ (for $d=1$ this statement was proven 
rigorously both for thin $[4,5]$ and thick $[6,7]$ disordered wires). It means that without inelastic processes $\sigma_{d=1,2}(T)=0$, while for $\sigma_{d=3}(T)$ one should expect the Arrhenius law. Note that for electrons in a crystal within a given conduction band the latter conclusion could become incorrect - strong enough disorder can localize the whole band.

Situation becomes more complicated when the inelastic processes are taken into account. In particular, electron-phonon interaction leads to the mechanism of conductivity known as hopping conductivity $[8,9]$ - with an assistance of phonons electrons hop between the localized states without being activated above the mobility edge. As a result, $\sigma(T)$ turns out to be finite (although it can be very small) at any finite $T$ even when all one-electron states are localized.

Can interaction between electrons play the same role and cause the hopping conductivity? This question was discussed in literature for a long time [1014] and no definite conclusion was achieved. The problem is that although the electric noise exists inside the material with a finite ac conductivity ${ }^{1}$ the "photons" in contrast with phonons become localized together with electrons.

In this paper we demonstrate that electron-electron interaction alone cannot cause finite conductivity even when temperature is finite, but small enough. In the absence of phonons and extended one-electron states a system of interacting electrons has exactly zero conductivity below some temperature $T_{c}$. This critical temperature is infinite if the distance between the electrons exceeds the localization lengths of all electronic states. In the opposite case $T_{c}$ turns out to be finite and depends on the typical number of electrons within the localization volume as well as on the strengths of the electron-electron interaction. We also argue that at high temperatures $T>T_{c}$ the conductivity $\sigma(T)$ is finite. It means that at $T=T_{c}$ the system of interacting electrons subject to a random potential undergoes a genuine phase transition that manifests itself by the emerging of a finite conductivity!

This transition can be thought of as many-body localization - it applies to many-body eigenstates of the whole system. This localization occurs not in the real space, but rather in the Fock space. This fact does not affect the validity of the concept of mobility edge. In fact, the existence of the "metallic" state at $T>T_{c}$ implies that the many-body states with energies $\mathcal{E}$ above $\mathcal{E}_{c}$ are extended. One can estimate the difference between $\mathcal{E}_{c}$ and the energy of the many-body ground state $\mathcal{E}_{0}$ as $\mathcal{E}_{c}-\mathcal{E}_{0} \sim T \mathcal{N}(T)$, where $\mathcal{N}(T)$ is the total number of one-particle states in the energy strip of the width $T$. Note that the existence of the extended many-body states above the mobility edge does not contradict the fact that below $T_{c}$ there is no conductivity - in contrast

$\overline{1}$ In this paper we mostly focus on dc conductivity. As to ac conductivity, it never vanishes, because at any frequency density of resonant pairs of states is finite. 
with the case of one-particle localization there is no Arrhenius regime since $\mathcal{E}_{c}-\mathcal{E}_{0}$ turns out to be proportional to the volume of the system, i.e., is macroscopically large (see Sec. 2.1.2 below for more details).

In order to avoid possible misunderstanding we would like to emphasize at the very beginning that throughout this paper we will be focused only on the inelastic collisions between the electrons, i.e., on creation or annihilation of real electron-hole pairs. There are other effects of electron-electron interactions - they can be understood as renormalization of the one-particle random potential by the interaction. This renormalization is temperature dependent and thus leads to a number of interesting effects, for example, the interaction corrections to the density of states and conductivity in disordered metals [15]. On the insulating side of the one-particle localization transition similar effects cause the well-known Coulomb gap [9] which suppresses hopping conductivity. On the other hand, this is still a time-independent correction to the timeindependent random potential. As such, it can maybe shift the position of the many-body Metal to Insulator transition, i.e., renormalize $T_{c}$, but is unable to destabilize the insulating or metallic phases. From now on we will simply neglect all elastic (Hartree-Fock) effects and concentrate on the real inelastic electron-electron collisions.

Localization of the many-body states in the Fock space has been discussed in Ref. [16] for the case of zero-dimensional systems with finite, although maybe large, number of electrons. Authors of Ref. [16] used an approximate mapping of the Hamiltonian of a metallic grain with large Thouless conductance $g$ and moderate interaction between the electrons to the one-particle Hamiltonian on a lattice with the topology of the Cayley tree and an on-site disorder. The latter problem has an exact solution [17] that contains the localization transition. In terms of interaction electrons this transition means that below certain energy of the excitation the one-particle excitation states are quite close to some exact many-body excitations. As to the one-particle excitations with energies higher than the critical one, its wave function can be viewed as a wave packet, which involves a large number of the many-body eigenstates. Being a property of a finite system this transition could be nothing but a crossover, which becomes more pronounced with increasing of $g$.

For an infinite system with $d>0$ the presence of spatial degrees of freedom makes the situation more complex. In this case, Cayley tree approximation turns out to be insufficient. Nevertheless, a consistent analysis of a model with weak and short range interaction to all orders of perturbation theory enabled us to analyze the many-body localization transition and to demonstrate that both the metallic state at high temperatures and the insulating state at low temperatures are stable and survive all higher loop corrections to the locator expansion. This allows us to claim that the existence of the transition is proven on the physical level of rigor. 
It should be noted that such an insulating state that is characterized by exactly zero conductivity is quite different from all other known types of Metal to Insulator transitions. For example, Mott insulator is believed to have finite, though exponentially small conductivity at finite temperatures.

The remainder of the paper is organized as follows. Sec. 2 contains the discussion of the problem on the qualitative level: we define the many-body localization, show its macroscopic implications, and discuss the relation to the Anderson model on a certain graph. In Sec. 3 we specify the model many-body system to be studied throughout the paper. In Sec. 4 we show how to treat this model in the framework of non-equilibrium Keldysh formalism, introduce the self-consistent Born approximation (SCBA), and derive the general equations. Using these equations, we demonstrate the existence of the metallic state at high temperatures and study its properties in Sec. 5. Sec. 6 is devoted to the proof of the existence of the insulating phase at low temperatures; we evaluate the transition temperature as the limit of stability of the insulating phase. Sec. 7 is dedicated to justification of SCBA; we demonstrate that corrections to SCBA are indeed small. Finally, in Sec. 8 we summarize the results and present an outlook of the future developments.

\section{Qualitative discussion}

\subsection{Macroscopic manifestations of the many-body localization transition}

\subsubsection{Single-particle localization}

Let us begin with the brief review of the basic notion emerged in a study of the properties of the one electron wave functions in a disordered potential in $d$ dimensions. Depending on the strength of the disorder potential, a wave function $\phi_{\alpha}(\boldsymbol{r})$ of an eigenstate $\alpha$ corresponding to a given energy can be classified as localized or extended:

$$
\left|\phi_{\alpha}(\boldsymbol{r})\right|^{2} \propto\left\{\begin{array}{cl}
\frac{1}{\zeta_{l o c}^{d}} \exp \left(-\frac{\left|\boldsymbol{r}-\boldsymbol{\rho}_{\alpha}\right|}{\zeta_{l o c}}\right) ; & \text { localized } \\
\frac{1}{\mathcal{V}} ; & \text { extended }
\end{array}\right.
$$

where $\zeta_{l o c}$ is the localization length which depends on the eigenenergy $\xi_{\alpha}$, and $\mathcal{V}$ is the volume of the system. Exponentially localized states have a maximum amplitude at some point $\boldsymbol{\rho}_{\alpha}$ in the system and a wave function envelope which falls off exponentially, whereas the extended states spread relatively uniformly over the whole volume of the system. It is believed that the coexistence of the localized and extended states in the same energy range is not possible, and the spectrum splits into bands of localized and extended 
states. The energies separating such bands are known as mobility edges. For example, for free electrons in a disorder potential in the dimensionality three and higher, only one mobility edge $\mathcal{E}_{1}$ exists, so that

$$
\begin{array}{ll}
\xi_{\alpha}<\mathcal{E}_{1}: & \text { localized; } \\
\xi_{\alpha}>\mathcal{E}_{1}: & \text { extended }
\end{array}
$$

The statement about the asymptotic behaviour of the single-particle wavefunctions (1) can be translated into the property of the matrix elements of a certain local operator $\hat{A}(\boldsymbol{R})$ (it might be the local mass or current density operator, etc.):

$$
A_{\alpha \beta}(\boldsymbol{R})=\int \mathrm{d}^{d} \boldsymbol{r} \phi_{\alpha}^{*}(\boldsymbol{r}) \hat{A}(\boldsymbol{R}) \phi_{\beta}(\boldsymbol{r})
$$

Then,

$$
\mathcal{L}_{\alpha \beta}^{A}(\boldsymbol{r})=\int \mathrm{d}^{d} \boldsymbol{R} A_{\alpha \beta}(\boldsymbol{R}) A_{\beta \alpha}(\boldsymbol{R}+\boldsymbol{r}) \propto\left\{\begin{array}{cc}
\leq \mathrm{e}^{-\frac{|\boldsymbol{r}|}{\zeta_{l o c}}} ; & \text { localized } \\
\mathcal{F}\left(\frac{|\boldsymbol{r}|}{L_{\omega_{\alpha \beta}}}\right) ; & \text { extended }
\end{array}\right.
$$

where the linear scale $L_{\omega}$ is controlled by the transmitted energy $\omega_{\alpha \beta}=\xi_{\alpha}-\xi_{\beta}$ only, and $L_{\omega} \rightarrow \infty$ for $\omega \rightarrow 0$. No summation over the repeating indices is implied in Eq. (4). Energies $\xi_{\alpha}$ and $\xi_{\beta}$ are assumed to be sufficiently close to each other, so that the localization length is approximately the same for the two states.

The notion of the matrix elements (4) is intimately related to the observable quantities. As an example, consider the Kubo formula for the density-density response function

$$
\Pi(\omega ; \boldsymbol{r})=\frac{1}{\mathcal{V}} \sum_{\alpha \beta} \frac{\left(f_{\alpha}-f_{\beta}\right) \mathcal{L}_{\alpha \beta}^{\varrho}(\boldsymbol{r})}{\omega-\xi_{\alpha}+\xi_{\beta}+i 0},
$$

where the overlap $\mathcal{L}_{\alpha \beta}^{\varrho}(\boldsymbol{r})$ is given by Eq. (4) built on the operators of the local density, $\hat{\varrho}(\boldsymbol{R})$, such that $\hat{\varrho}(\boldsymbol{R})|\boldsymbol{r}\rangle=\delta(\boldsymbol{R}-\boldsymbol{r})|\boldsymbol{r}\rangle$. In the thermal equilibrium for the fermionic particles, the occupation numbers $f_{\alpha}$ are given by the Fermi distribution.

In a conducting system, the low-frequency asymptotic behavior of the densitydensity response function is always determined by diffusion as the total number of particle is the only conserved quantity in the system:

$$
\Pi(\omega ; \boldsymbol{r}) \propto \int \mathrm{d}^{d} \boldsymbol{Q} \frac{\mathrm{e}^{i \boldsymbol{Q} \cdot \boldsymbol{r}}}{-i \omega+D \boldsymbol{Q}^{2}},
$$


where $D$ is the diffusion coefficient. First, let assume that all the states are localized. Then, comparing Eq. (5) with Eq. (4), we see that the asymptotic behaviour of the wave function precludes the diffusion propagation (6). Therefore, the diffusion coefficient vanishes for any temperature $T$. The same is true for the dc conducivity, as it is related to the diffusion coefficient via Einstein relation, $\sigma \propto D$. Physical meaning of vanishing $D$ and $\sigma$ is the impossiblity for an excitation caused by a local external perturbation to propagate in a localized system, and uniformly span all the phase space allowed by the conservation of energy.

If a finite mobility edge (2) exists and the Fermi level $\epsilon_{F}$ lies in the energy region of localized states, the conducitivity is determined by the exponentially small occupation number of the delocalized states

$$
\sigma(T) \propto e^{-\left(\mathcal{E}_{1}-\epsilon_{F}\right) / T} .
$$

In this paper we will be interested in transport properties of the systems where all single-particle states are localized, and thus without many-body effects $\sigma=0$ at any temperature. It is well established now that the mobility edge does not exist for one- and two-dimensional systems for any disorder, and all single-particle states are indeed localized. Such a situation can also arise in an arbitrary high dimensionality as well. Indeed, consider as an example Anderson model with one state per lattice site [1]. It is well known that all eigenstates become localized as soon as the on-site disorder exceeds a critical value.

\subsubsection{Many-body localization}

Let us now turn to the discussion of the many-body localization. From now on, we assume that all one-particle state are localized in the sense of the previous subsection. Can the interaction cause finite conductivity? Consider a many-body eigenstate $\left|\Psi_{k}\right\rangle$ of the interacting system, with the corresponding eigenenergy $E_{k}$. Our purpose is to generalize the notion of localization to such many-body states.

In the coordinate representation, the many-body wave function $\Psi_{k}\left(\left\{\boldsymbol{r}_{j}\right\}_{j=1}^{N}\right)$ depends on the coordinates of all $N$ particles in the system. The single-particle states forming this many-body state are located everywhere in the volume $\mathcal{V}$. Thus, no definition analogous to Eq. (1) can be constructed. On the other hand, the relation (4) can be used, if one takes a local additive one-particle operator:

$$
\hat{\mathbb{A}}(\boldsymbol{R})=\sum_{\alpha \beta} A_{\alpha \beta}(\boldsymbol{R}) \hat{c}_{\alpha}^{\dagger} \hat{c}_{\beta} \equiv \int \mathrm{d}^{d} \boldsymbol{r} \hat{c}^{\dagger}(\boldsymbol{r}) \hat{A}(\boldsymbol{R}) \hat{c}(\boldsymbol{r}),
$$

where we introduced the fermionic creation and annihilation operators $\hat{c}_{\alpha}^{\dagger}$, $\hat{c}^{\dagger}(\boldsymbol{r}), \hat{c}_{\alpha}, \hat{c}(\boldsymbol{r})$ in the basis of the one-particle eigenstates and in the coordinate 
representation, respectively.

We consider the matrix elements of the local operator between two exact many-body eigenstates:

$$
\mathbb{A}_{k k^{\prime}}(\boldsymbol{R})=\left\langle\Psi_{k}|\hat{\mathbb{A}}(\boldsymbol{R})| \Psi_{k^{\prime}}\right\rangle
$$

Then we can define localized states by a relation, analogous to Eq. (4):

$$
\mathcal{L}_{k k^{\prime}}^{\mathbb{A}}(\boldsymbol{r})=\int \mathrm{d}^{d} \boldsymbol{R} \mathbb{A}_{k k^{\prime}}(\boldsymbol{R}) \mathbb{A}_{k^{\prime} k}(\boldsymbol{R}+\boldsymbol{r}) \propto\left\{\begin{array}{l}
\leq \mathrm{e}^{-\frac{|\boldsymbol{r}|}{\zeta\left(E_{k}\right)}} ; \text { localized } \\
\mathcal{F}\left(\frac{|\boldsymbol{r}|}{L_{\omega_{k k^{\prime}}}}\right) ; \text { extended }
\end{array}\right.
$$

Again, $\omega_{k k^{\prime}}=E_{k}-E_{k^{\prime}}$ and the energies $E_{k}$ and $E_{k^{\prime}}$ are taken sufficiently close to each other, so that the difference between $\zeta\left(E_{k}\right)$ and $\zeta\left(E_{k^{\prime}}\right)$ need not be taken into account. ${ }^{2}$

Equation (10) is the definition of the localized many-body states. To elucidate its meaning, let calculate the matrix element (9) between two Hartree-Fock states

$$
\left|\Psi_{k}^{H F}\right\rangle=\prod_{\alpha}\left(1-f_{\alpha}^{k}+f_{\alpha}^{k} c_{\alpha}^{\dagger}\right)|\operatorname{vacuum}\rangle,
$$

where the state $\left|\Psi_{k}^{H F}\right\rangle$ is completely characterized by the set of occupation numbers $f_{\alpha}^{k}=0,1$, corresponding to empty and filled single-particle states, respectively. Substituting Eq. (11) into Eq. (8) we find for $k \neq k^{\prime}$

$$
\mathbb{A}_{k k^{\prime}}=\sum_{\alpha \beta} A_{\alpha \beta}\left[f_{\alpha}^{k}\left(1-f_{\alpha}^{k^{\prime}}\right) f_{\beta}^{k^{\prime}}\left(1-f_{\beta}^{k}\right)\right] \prod_{\gamma \neq \alpha, \beta} \delta_{f_{\gamma}^{k}, f_{\gamma}^{k^{\prime}}}
$$

where we omitted the argument $\boldsymbol{R}$ in both sides of the equation. The states $k$ and $k^{\prime}$ connected by the operator $\hat{\mathbb{A}}(\boldsymbol{R})$ are obtained from each other by creation of a single electron-hole pair, so that only one term in the sum over $\alpha$ and $\beta$ is different from zero. We note that the matrix elements $A_{\alpha \beta}(\boldsymbol{R})$ are exponentially suppressed unless both states $\alpha$ and $\beta$ are located near the point $\boldsymbol{R}$. Thus, the distance between the electron and the hole cannot exceed the single-particle localization length $\zeta_{l o c}$, and the number of states $k^{\prime}$ which can be connected to the given state $k$ by the local perturbation is effectively finite, even though the total number of the Slater determinat states (11) scales exponentially with the size of the system.

${ }^{2}$ Let us emphasize that the criterion (9) can be extended to the arbitrary number of electron-hole excitations, $\hat{A}(\boldsymbol{R}) \rightarrow \hat{A}_{1}(\boldsymbol{R}) \hat{A}_{2}(\boldsymbol{R}) \ldots \hat{A}_{n}(\boldsymbol{R})$, corresponding to the local heating of the system. The asymptotic behaviour will still be determined by Eq. (10). 
We substitute Eq. (12) into the localization criterion (10) and obtain

$$
\mathcal{L}_{k k^{\prime}}^{\mathbb{A}}(\boldsymbol{r})=\sum_{\alpha \beta} \mathcal{L}_{\alpha \beta}^{A}(\boldsymbol{r})\left[f_{\alpha}^{k}\left(1-f_{\alpha}^{k^{\prime}}\right) f_{\beta}^{k^{\prime}}\left(1-f_{\beta}^{k}\right)\right] \prod_{\gamma \neq \alpha, \beta} \delta_{f_{\gamma}^{k}, f_{\gamma}^{k^{\prime}}}
$$

Because of the $\delta$-symbols, $\left|E_{k}-E_{k^{\prime}}\right|=\left|\sum_{\gamma} \xi_{\gamma}\left(f_{\gamma}^{k}-f_{\gamma}^{k^{\prime}}\right)\right|=\left|\xi_{\alpha}-\xi_{\beta}\right|$, where $\alpha$ and $\beta$ are the only two states contributing to the sum for given $k, k^{\prime}$. If $\xi_{\alpha}, \xi_{\beta}$ are not too far from the Fermi level, so that $\zeta_{l o c}\left(\xi_{\alpha}\right) \approx \zeta_{l o c}\left(\xi_{\beta}\right)$, we find that $\mathcal{L}_{k k^{\prime}}^{\mathbb{A}}(\boldsymbol{r})$ has the same spatial dependence as the kernel (4) for noninteracting system. Thus, according to definition (10) any Hartree-Fock state (11) is localized with the localization length $\zeta\left(E_{k}\right)=\zeta_{l o c}$.

Let us apply the same ideas to the Kubo formula for the many-body densitydensity response function

$$
\begin{aligned}
& \Pi(\omega ; \boldsymbol{r})=\sum_{k} P_{k} \Pi_{k}(\omega, \boldsymbol{r})=\sum_{k k^{\prime}}\left[P_{k}-P_{k^{\prime}}\right] \Pi_{k k^{\prime}}(\omega, \boldsymbol{r}) \\
& \Pi_{k}(\omega, \boldsymbol{r})=\sum_{k^{\prime}}\left[\Pi_{k k^{\prime}}(\omega, \boldsymbol{r})-\Pi_{k^{\prime} k}(\omega, \boldsymbol{r})\right] \\
& \operatorname{Im}_{k k^{\prime}}(\omega, \boldsymbol{r})=\frac{\pi}{\mathcal{V}} \mathcal{L}_{k k^{\prime}}^{\varrho}(\boldsymbol{r}) \delta\left(\omega+E_{k}-E_{k^{\prime}}\right)
\end{aligned}
$$

where $P_{k}$ is the probability for the system to be in the eigenstate $k$ and $\Pi_{k}(\omega, \boldsymbol{r})$ is the linear response of the system in this particular eigenstate. The overlap function $\mathcal{L}_{k k^{\prime}}^{\varrho}(\boldsymbol{r})$ is given by Eq. (10) with the operator of local density $\hat{\varrho}(\boldsymbol{R})=\hat{c}^{\dagger}(\boldsymbol{R}) \hat{c}(\boldsymbol{R})$.

Formulas (14) are valid for any many-body eigenstates $\left|\Psi_{k}\right\rangle$. For the HartreeFock state (11), we can substitute Eq. (13) into Eqs. (14) and obtain Eq. (5) with

$$
f_{\alpha}=\sum_{k} P_{k} f_{\alpha}^{k}
$$

For the initial Gibbs distribution

$$
P_{k}=\frac{\exp \left(-\frac{E_{k}}{T}\right)}{\sum_{k} \exp \left(-\frac{E_{k}}{T}\right)}
$$

and the Hartree-Fock spectrum, $E_{k}=\sum_{\alpha} f_{\alpha}^{k} \xi_{\alpha}$, formula (15) gives the Fermi distribution function.

According to Eqs. (13) and (14c), the spatial dependence of the correlator $\Pi(\omega, \boldsymbol{r})$ is still given by Eq. (4) for the localized single-particle wave functions. Therefore, both the conductivity and the diffusion coefficient vanish for the Hartree-Fock states constructed in the basis of exact one-particle wave functions of disordered systems. The same statement can be made about certain 
wave functions which are formally very different from a single Slater determinant. Namely, consider the creation of the electron-hole pair on top of some eigenstate $\left|\Psi_{k}\right\rangle$ and expand the result in terms of other eigenstates

$$
\hat{c}_{\alpha}^{\dagger} \hat{c}_{\beta}\left|\Psi_{k}\right\rangle=\sum_{k^{\prime}} C_{\alpha \beta}^{k k^{\prime}}\left|\Psi_{k^{\prime}}\right\rangle ; \quad \sum_{k^{\prime}}\left|C_{\alpha \beta}^{k k^{\prime}}\right|^{2}=1
$$

For the Hartree-Fock state (11), only one term contributes to the sum, see Eq. (13). It is possible to check that the state would remain localized in a sense of Eq. (10), if the number of terms contributing to the sum in Eq. (17a) is large but finite, i.e.

$$
\lim _{\mathcal{V} \rightarrow \infty}\left[\sum_{k^{\prime}}\left|C_{\alpha \beta}^{k k^{\prime}}\right|^{4}\right]^{-1}<\infty .
$$

In complete analogy with the non-interacting system, this corresponds to the insulating or localized many-body state; excitation can not propagate over all states allowed by the energy conservation.

Conductivity can be different from zero only if the wave functions of the excitations can be broken onto the infinite number of eigenstates

$$
\lim _{\mathcal{V} \rightarrow \infty}\left[\sum_{k^{\prime}}\left|C_{\alpha \beta}^{k k^{\prime}}\right|^{4}\right]^{-1}=\infty,
$$

which would correspond to the metallic or extended many-body state.

Developed metallic state corresponds to the case when electron-electron interaction mixes the excited state with all the eigenstates in the system with close enough energy:

$$
\left|C_{\alpha \beta}^{k k^{\prime}}\right|^{2} \propto " \delta\left(E_{k}+\omega_{\alpha \beta}-E_{k^{\prime}}\right) ",
$$

where $\delta$-function should be understood in the thermodynamic sense: its width, although sufficiently large to include many states, vanishes in the limit $\mathcal{V} \rightarrow$ $\infty$. Only in this regime, which may also be called ergodic many-body state, the electron-electron interaction can bring the system from the intitial HartreeFock state to the equilibrium correspoding to spanning all the states permitted by the energy conservation. In this case, the averaging over the exact manybody eigenfunction is equivalent to averaging over the microcanonical distribution, and temperature $T$ can be defined as a usual Lagrange multiplier. It is related to $E_{k}$ by the thermodynamic relation:

$$
E_{k}-E_{0}=\int_{0}^{T} C_{V}\left(T_{1}\right) \mathrm{d} T_{1}
$$

where $E_{0}$ is the ground state energy, and $C_{V}(T) \propto \mathcal{V}$ is the specific heat. 
The main result obtained in the present paper is the proof of existence of the extensive many-body mobility edge $\mathcal{E}_{c} \propto \mathcal{V}$. This proof is based on two statements: (i) states with sufficiently large energies $E_{k}-E_{0}$ are extended; (ii) states with sufficiently small energies $E_{k}-E_{0}$ are localized.

The first statement follows from the validity of high-temperature expansion for the quantum corrections to conductivity [15]. The quantum corrections to conductivity are divergent for $d=1,2$; for non-interacting systems these weak localization corrections have no cutoff other than the size of the system. In interacting systems two kinds of new phenomena appear: (a) interference due to the scattering off the self-consistent Hartree-Fock potential (see Ref. [18]), and (b) inelastic electron scattering. Effects of type (a) are regularized by the temperature itself and do not produce any consequences for the present paper. At the same time, inelastic electron scattering leads to regularization of the weak localization corrections due to appearence of the inelastic rate and the corresponding length:

$$
\frac{1}{\tau_{\phi}} \simeq \frac{\lambda^{2} T}{g\left(L_{\phi}\right)}, \quad L_{\phi} \simeq \sqrt{D \tau_{\phi}},
$$

where $\lambda \lesssim 1$ is the dimensionless interaction constant, $D$ is the diffusion coefficient, and $g(L)$ is the dimensionless conductance of the $d$-dimensional cube of linear size $L$. All the interference corrections are finite if

$$
\frac{1}{\tau_{\phi}} \gtrsim \delta_{\zeta} .
$$

Using $g\left(\zeta_{\text {loc }}\right) \simeq 1$, we rewrite Eqs. (19) and (20) as

$$
T \gtrsim T^{(e l)} \simeq \frac{\delta_{\zeta}}{\lambda^{2}} .
$$

Inequality (21) is the condition of applicability of the expansion from the metallic state. In fact, it is also valid for higher-dimensional systems with the finite bandwidth $E_{b}$ and all single-particle eigenstates localized, if $E_{b} \gg T^{(e l)}$.

At $T<T_{e l}$ the perturbation theory breaks down. It may indicate either (i) a simple insufficiency of the perturbation theory to describe the metallic state, or (ii) existence of the many-body mobility edge $\mathcal{E}_{c}$. It important to emphasize that $\mathcal{E}_{c}$ is an extensive quantity $\mathcal{E}_{c} \propto \mathcal{V}$.

In case (i), states $\Psi_{k}$ are extended for all $E_{k}$, so that the conductivity would remain finite (no matter how small) down to zero temperature. In case (ii) the many-body eigenstates with $E_{k}-E_{0} \leq \mathcal{E}_{c}$ are localized, see Eq. (17b).

In case (ii), the partial conductivity of one state, $\sigma_{k}$, defined analogously to $\Pi_{k}$ from Eqs. (14), is zero for $E_{k}-E_{0} \leq \mathcal{E}_{c}$. 


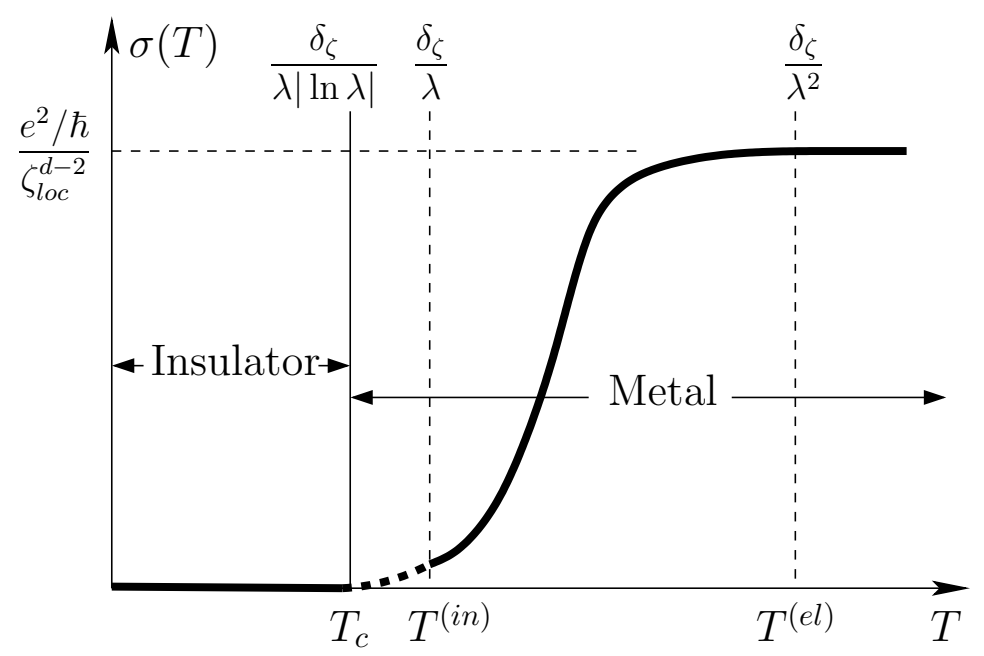

Fig. 1. Schematic temperature dependence of the dc conductivity $\sigma(T)$. Below the point of the many-body metal-insulator transition, $T<T_{c}, \sigma(T)=0$, as shown in Sec. 6. Temperature interval $T>T^{(i n)}>T_{c}$ corresponds to the developed metallic phase, where Eq. (17d) is valid. In this regime for the model described in Sec. 3 $\sigma(T)$ is given analytically by Eqs. (93)-(99) and plotted on Fig. 10. At $T>T^{(e l)}$ the high-temperature metallic perturbation theory of Ref. [15] is valid.

Results of Ref. [16] strongly indicate that the case (ii) is realized. We will review, extend and refine the arguments of Ref. [16] in the next subsection; here, we simply proceed with the discussion of the macroscopic manifestations of this scenario. Let us assume that the equilibrium occupation is given by the Gibbs distribution (16). One could think that it would still imply the Arrhenius law (7) for the conductivity. However, this is not the case for the many-body mobility threshold. In fact, in the limit $\mathcal{V} \rightarrow \infty$

$$
\sigma(T)=0 ; \quad T<T_{c}
$$

where the critical temperature is determined by Eq. (18):

$$
\int_{0}^{T_{c}} \mathrm{~d} T_{1} C_{V}\left(T_{1}\right)=\mathcal{E}_{c}
$$

The schematic temperature dependence of the conductivity is summarized on Fig. 1. Therefore, the temperature dependence of the dissipative coefficient in the system shows the singularity typical for a phase transition.

To prove Eqs. (22) we use the Gibbs distribution and find

$$
\sigma(T)=\sum_{k} P_{k} \sigma\left(E_{k}\right)=\frac{\int_{0}^{\infty} \mathrm{d} E \mathrm{e}^{S(E)-E / T} \sigma(E)}{\int_{0}^{\infty} \mathrm{d} E \mathrm{e}^{S(E)-E / T}},
$$

where the entropy $S(E)$ is proportional to volume, and $E$ is counted from the ground state. The integral is calculated in the saddle point or in the steepest 
decent approximations, exact for $\mathcal{V} \rightarrow \infty$. The saddle point $E(T)$ is given by

$$
\left.\frac{d S}{d E}\right|_{E=E(T)}=\frac{1}{T}
$$

Taking into account $\sigma(E)=0$ for $E<\mathcal{E}_{c}$ we find

$$
\begin{aligned}
& \sigma(T)=\sigma[E(T)], \quad E(T)>\mathcal{E}_{c} ; \\
& \sigma(T) \propto \exp \left(-\frac{\mathcal{E}_{c}-E(T)}{T}\right) ; \quad E(T)<\mathcal{E}_{c}
\end{aligned}
$$

As both energies entering the exponetial are extensive, $E(T), \mathcal{E}_{c} \propto \mathcal{V}$, we obtain Eqs. (22).

As we already mentioned, vanishing of the dissipative conductivity at $T<T_{c}$ means freezing of all relaxation processes. In particular the microcanonical distribution could never be established for the closed system. In this respect, the dynamics of the system resembles the glassy state [19].

To establish the thermal equilibrium in such insulating state requires finite coupling of the system with the external reservoir (i.e., phonons). The presence of the finite electron-phonon interaction (as phonons are usually delocalized), smears out the transition, and $\sigma(T)>0$ for any temperature. Nevertheless, for the weak electron-phonon interaction, the phenomenon of the many-body metal-insulator transition (22) manifests itself as a very sharp crossover from phonon induced hopping at $T<T_{c}$ to the conductivity independent of the electron-phonon coupling at $T>T_{c}$.

\subsection{Microscopic mechanism of the many-body localization transition}

As discussed in the previous subsection, the existence of extended many-body states at high energies is an established fact [15]. Here we focus on the existence of localized states at low energies, and discuss the correspondence between a many-electron interacting system and the Anderson model on a certain graph. As we have already mentioned, it is convenient to analyze many-body localization in terms of single-particle excitations $\hat{c}_{\alpha}^{\dagger}\left|\Psi_{k}\right\rangle$ above a certain eigenstate of the interacting system, namely, how these excitations spread over other many-bogy eigenstates (consideration of the electron-hole excitation is performed in a same fashion and does not bring anything qualitatively different). This discussion has a close relation with that of Ref. [16].

Let the system initially be in the eigenstate $\left|\Psi_{k}\right\rangle$. At time $t=0$ an extra electron is created in the single-particle state $\alpha$. The many-body Schrödinger 
equation describing the subsequent time evolution of such state $\left|\tilde{\Psi}_{k \alpha}(t)\right\rangle$ is

$$
\left[i \partial_{t}-\hat{H}\right]\left|\tilde{\Psi}_{k \alpha}(t)\right\rangle=\delta(t) \hat{c}_{\alpha}^{\dagger}\left|\Psi_{k}\right\rangle
$$

where the right-hand side determines the initial condition at $t=0$, and the Hamiltonian, written in the basis of the exact single electron wavefunctions, is given by

$$
\hat{H}=\sum_{\alpha} \xi_{\alpha} \hat{c}_{\alpha}^{\dagger} \hat{c}_{\alpha}+\frac{1}{2} \sum_{\alpha \beta \gamma \delta} V_{\alpha \beta \gamma \delta} \hat{c}_{\alpha}^{\dagger} \hat{c}_{\beta}^{\dagger} \hat{c}_{\gamma} \hat{c}_{\delta}
$$

The interaction part of the Hamiltonian contains only non-diagonal terms, $\alpha \neq \gamma, \delta ; \beta \neq \gamma, \delta$, and assumed to be antisymmetrized, $V_{\alpha \beta \gamma \delta}=-V_{\beta \alpha \gamma \delta}=$ $-V_{\alpha \beta \delta \gamma}$. The diagonal matrix elements are already included into the definition of the Hartree-Fock spectrum $\left\{\xi_{\alpha}\right\}$.

We make the Fourier transform of Eq. (23):

$$
\left(\epsilon+E_{k}-\hat{H}\right)\left|\tilde{\Psi}_{k \alpha}(\epsilon)\right\rangle=\hat{c}_{\alpha}^{\dagger}\left|\Psi_{k}\right\rangle
$$

(here we count the energy $\epsilon$ from that of the reference state $E_{k}$ ), and solve this equation for $\left|\tilde{\Psi}_{k \alpha}(\epsilon)\right\rangle$ by iterations:

$$
\left|\tilde{\Psi}_{k \alpha}(\epsilon)\right\rangle=\frac{1}{\epsilon-\xi_{\alpha}}\left(\left|\psi_{k \alpha}^{(0)}(\epsilon)\right\rangle+\left|\psi_{k \alpha}^{(1)}(\epsilon)\right\rangle+\ldots\right) .
$$

The zeroth order in the electron-electron interaction is just the one-particle excitation itself:

$$
\left|\psi_{k \alpha}^{(0)}\right\rangle=\hat{c}_{\alpha}^{\dagger}\left|\Psi_{k}\right\rangle
$$

The first order corresponds to a three-particle excitation above $\left|\Psi_{k}\right\rangle$ :

$$
\left|\psi_{k \alpha}^{(1)}\right\rangle=\sum_{\beta, \gamma, \delta} \frac{V_{\delta \gamma \beta \alpha}}{\epsilon-\Xi_{\gamma \delta}^{\beta}} \hat{c}_{\delta}^{\dagger} \hat{c}_{\gamma}^{\dagger} \hat{c}_{\beta}\left|\Psi_{k}\right\rangle,
$$

where we introduced a shorthand notation

$$
\Xi_{\gamma \delta}^{\beta}=\xi_{\gamma}+\xi_{\delta}-\xi_{\beta}
$$

for the energy of the three-particle excitation.

As usual in the theory of metal-insulator transition [1], one starts from estimating the probability for the sum (28) not to be small. We notice that the geometric distance between particles is of the order of the one-particle localization length $\zeta_{l o c}$, as the interaction is short-ranged. Thus, the denominators in Eq. (28) are the random quantities with the maximal value of the order of the level spacing in the localization length $\delta_{\zeta}$. Assuming weak interaction, $\lambda=\max \left|\mathrm{V}_{\alpha \beta \gamma \delta}\right| / \delta_{\zeta} \ll 1$, we see that the value of sum is, in fact, determined by the smallest denominator. 
Let us assume that the typical number of terms (i.e., terms not suppressed by the matrix elements) is $K \gg 1$. As the denominators are distrubited approximately uniformly for $-\delta_{\zeta}<\epsilon-\Xi_{\gamma \delta}^{\beta}<\delta_{\zeta}$, the smallest denominator is of the order of $\delta_{\zeta} / K$. Therefore, if $K \lambda \ll 1$, the probability to find the large mixing is small, whereas for

$$
K \lambda \gtrsim 1
$$

it becomes of the order of unity. This estimate up to a factor of the order of $|\ln \lambda|$ is the basis for finding the position of the transition [1].

The remaining non-trivial problems are: (i) to find the connectivity $K$, and (ii) to check that higher-order terms indeed match the locator structure of Anderson, i.e., the the number $\mathcal{N}_{n}$ of the $n$th order terms scales as

$$
\mathcal{N}_{n} \simeq K^{n},
$$

where the prefactor can be any algebraic function of $n$. Condition (31) is very important: if $\mathcal{N}_{n} \gg K^{n}, e . g ., \mathcal{N}_{n} \simeq n$ !, then the probability to find small enough denominators in high orders of the perturbation theory $\mathcal{N}_{n} \lambda^{n} \simeq 1$ would be always of the order of unity no matter how small the interaction constant $\lambda$ is; the system in this case would always remain metallic. If, oppositely, the number of terms in high orders is small, e.g., $\mathcal{N}_{n} \simeq K^{n} / n$ !, the higher-order terms will not contain the small denominators, even though the lowest orders of the perturbation theory do. In this case, the system would always remain insulating. In both cases, the conlusions drawn from the lowest order perturbation theory [16] would be misleading.

To estimate $K$ from Eq. (28), one has to account for (i) the structure of the eigenfunction $\left|\Psi_{k}\right\rangle$, i.e., which indices $\alpha$ of fermionic operators $\hat{c}_{\alpha}$ are allowed not to produce zero result after action on the wave function; (ii) the structure of the matrix elements $V_{\alpha \beta \gamma \delta}$.

The energy of a typical Hartree-Fock state is almost uniformly distributed among the localization volumes. The fact that the eigenstate $\left|\Psi_{k}\right\rangle$ is a linear combination of an infinite number of Hartree-Fock states does not affect this assumption. Therefore, the state $\left|\Psi_{k}\right\rangle$ is contributed by electron-like and holelike excitations with the energy smaller than $T$, where $T$ is of the order of temerature given by Eq. (18). Considering the action of the fermionic operators on such a state we estimate

$$
-T<\xi_{\gamma}, \xi_{\delta} ; \quad \xi_{\beta}<T .
$$

Second restriction is based on the structure of the matrix elements. As we already mentioned, they restrict the final states to be at the distance not exceeding the localization length $\zeta_{l o c}$ from each other. In addition to this spatial restriction, the matrix elements decrease rapidly when the energy difference, 
say $\xi_{\alpha}-\xi_{\gamma}$, exceeds the level spacing $\delta_{\zeta}$, i.e., $V_{\alpha \beta \gamma \delta} \simeq \lambda \delta_{\zeta}$ only for

$$
\left|\xi_{\alpha}-\xi_{\delta}\right|,\left|\xi_{\beta}-\xi_{\gamma}\right| \lesssim \delta_{\zeta} \quad \text { or } \quad\left|\xi_{\alpha}-\xi_{\gamma}\right|,\left|\xi_{\beta}-\xi_{\delta}\right| \lesssim \delta_{\zeta}
$$

and vanish rapidly otherwise.

Counting the number of states $\beta, \gamma, \delta$ within one localization volume, such that they produce the value of the denominator in Eq. (28) smaller than $\delta_{\zeta}$. and satisfy both restrictions $(32)-(33)$, we obtain for $T \gg \delta_{\zeta}^{3}$

$$
K \simeq \frac{T}{\delta_{\zeta}}
$$

Comparing Eq. (34) with the estimate (30) we conclude that at $T \gtrsim \frac{\delta_{\zeta}}{\lambda}$ the first correction to the wavefunction (28) contains at least one term of the order of unity, which may signify the transition in the system.

To check that this conclusion is not an artefact of the lowest order perturbation theory, one has to analyze further terms in the expansion (26). The second order correction is given by

$$
\begin{aligned}
\left|\psi_{k \alpha}^{(2)}\right\rangle & =\sum_{\alpha_{1} \beta_{1}} \sum_{\beta, \gamma, \delta} \frac{V_{\alpha_{1} \beta_{1} \gamma \delta}}{\epsilon-\Xi_{\alpha_{1} \beta_{1}}^{\beta}} \frac{V_{\delta \gamma \beta \alpha}}{\epsilon-\Xi_{\gamma \delta}^{\beta}} \hat{c}_{\alpha_{1}}^{\dagger} \hat{c}_{\beta_{1}}^{\dagger} \hat{c}_{\beta}\left|\Psi_{k}\right\rangle \\
& +\sum_{\alpha_{1}, \beta_{1}, \gamma_{1}} \sum_{\beta, \gamma, \delta} \frac{2 V_{\alpha_{1} \beta_{1} \gamma_{1} \delta}}{\epsilon-\Xi_{\gamma \alpha_{1} \beta_{1}}^{\beta \gamma_{1}}} \frac{V_{\delta \gamma \beta \alpha}}{\epsilon-\Xi_{\gamma \delta}^{\beta}} \hat{c}_{\alpha_{1}}^{\dagger} \hat{c}_{\beta_{1}}^{\dagger} \hat{c}_{\gamma}^{\dagger} \hat{c}_{\beta} \hat{c}_{\gamma_{1}}\left|\Psi_{k}\right\rangle \\
& +\sum_{\alpha_{1}, \gamma_{1}, \delta_{1}} \sum_{\beta, \gamma, \delta} \frac{V_{\alpha_{1} \beta \gamma_{1} \delta_{1}}}{\epsilon-\Xi_{\gamma \delta \alpha_{1}}^{\gamma_{1} \delta_{1}}} \frac{V_{\delta \gamma \beta \alpha}}{\epsilon-\Xi_{\gamma \delta}^{\beta}} \hat{c}_{\alpha_{1}}^{\dagger} \hat{c}_{\delta}^{\dagger} \hat{c}_{\gamma}^{\dagger} \hat{c}_{\beta_{1}} \hat{c}_{\gamma_{1}}\left|\Psi_{k}\right\rangle,
\end{aligned}
$$

where the $(2 n+1)$-particle energies are defined as

$$
\Xi_{\alpha_{1} \ldots \alpha_{n+1}}^{\beta_{1} \ldots \beta_{n}}=\sum_{i=1}^{n+1} \xi_{\alpha_{i}}-\sum_{i=1}^{n} \xi_{\beta_{i}} .
$$

The first term in Eq. (35) is again a three-particle excitation. We can estimate its typical value as $\lambda^{2} T$, which is smaller than the first-order contribution (28) by a factor $\lambda \ll 1$; similar type terms arise also from the second and the third lines for $\hat{c}^{\dagger}, \hat{c}$ with coinciding indices. The actual meaning of this terms is the renormalization of the value of the two-particle interaction strength. They are analyzed with more rigor in Sec. 7.1, here we simply neglect them as they do not affect the statement about the transition.

Once again, we estimate the number of the relevant terms in the multiple sum in the last two lines Eq. (35). They are shown pictorially on Fig. 2b.

$\overline{3}$ The estimate of Ref. [20] for the quantum dot at $T=0$ is $K \simeq \epsilon^{2} / \delta^{2}$, where $\delta$ is the level spacing in the quantm dot. Our estimate is different because the energy restriction on the matrix elements (33) is absent in a quantum dot. 
a)

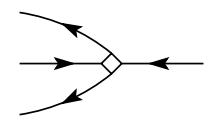

b)

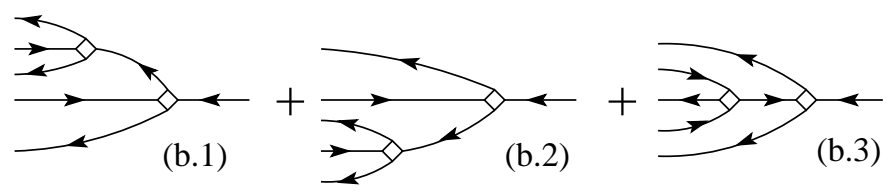

c)

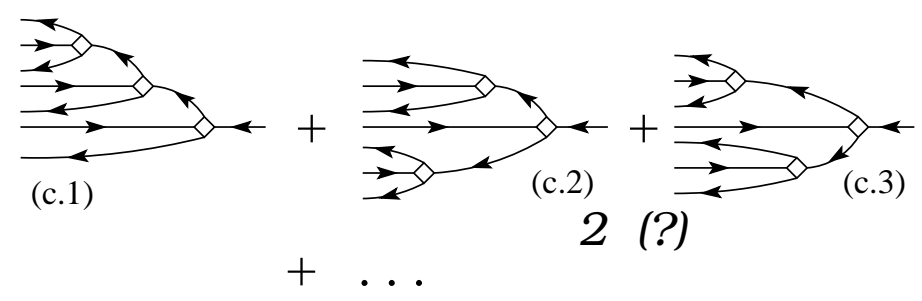

d) $2 n+1\left\{\begin{array}{c}- \\ \vdots \\ \vdots\end{array}\right\}$

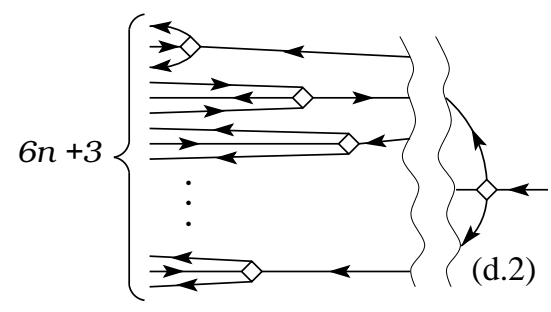

$(2 n+1) !(?)$

Fig. 2. Pictorial representation of the paths corresponding to the first (a), second (b), third (c), and higher (d) orders of the perturbation theory for the mixing of one-particle excitations with many-particle states. Process (d.2) is obtained from the process (d.1) by the application of the interaction operators once to each of $(2 \mathrm{n}+1)$ final particles of the process (d.1). The sequence of such applications can be ordered in $(2 n+1)$ ! ways, each producing formally different path.

The structure of the state $\left|\Psi_{k}\right\rangle$ gives the restriction similar to Eq. (32) on the electron (entering Eq. (36) with plus sign) and the hole (entering Eq. (36) with minus sign) energies respectively. The matrix element $V_{\alpha \beta \gamma \delta}$ restricts the energies by Eq. (33). Combining those two restrictions and requiring each denominator in Eq. (35) to be smaller than the level spacing $\delta_{\zeta}$, one estimates

$$
\mathcal{N}_{2} \simeq K^{2}
$$

where $K$ is given by Eq. (30). Aparently, Eq. (37) agrees with the requirement (31). However some questions may arise already on this stage.

One notices from Eq. (35) that there are terms which can be obtained from each other by the simple permutation of indices of the creation or annihilation 
operators. The states obtained by such permutations from each other are in fact identical, so one may be tempted to amend Eq. (37) as [20]

$$
\mathcal{N}_{n} \stackrel{?}{\simeq} \frac{K^{n}}{n !(n+1) !},
$$

where the combinatorial factors describe the indistinguishability among $n+1$ electrons and $n$ holes $[n=2$ for Eq. (35)].

However, in the theory of Anderson transition [1], the relevant parameter is not the number of available final states, but the number of statistically independent paths leading to these states from the initial state (e.g., for the Anderson transition in three dimensions the number of states grows as $n^{3}$ whereas the number of paths grows exponentially with $n$ ). By construction, the summation in Eq. (35) is performed over the single-particle indices and not over the final states. Moreover, the denominators in each terms involve different combinations of levels and thus produce statisitically independent contributions. This corresponds to the summation over paths number of which cancels the factorials in Eq. (38).

Having demonstrated the absence of the factorial factors suppressing the number of terms $\mathcal{N}_{n}$, one may worry about the opposite extreme: factorial growth of $\mathcal{N}_{n}$. The way to obtain this factorial is shown on Fig. 2 d. The transition between the two states with $(2 n+1)$ excitations and $(6 n+3)$ excitations may be realized in $(2 n+1)$ ! ways. These ways are different by the order in which interaction operator acts on the particles, thereby producing different intermediate states. If these paths were statistically independent, it would mean

$$
\mathcal{N}_{3 n+1} \stackrel{?}{\simeq}(2 n+1) ! K^{2 n+1} \mathcal{N}_{n}
$$

so that no transition would occur and the system would always be delocalized.

However, such paths are, in fact, correlated and the sum of the corresponding amplitudes produces always the result of the order of an amplitude of a single path; the terms essentially cancel each other. To demonstrate this cancellation we consider the third order of the perturbation theory. The terms schematically shown on Fig. 2c correspond to

$$
\begin{aligned}
\delta\left|\psi_{k \alpha}^{(3)}\right\rangle= & \sum_{\alpha_{2}, \beta_{2}, \gamma_{2}} \sum_{\alpha_{1}, \beta_{1}, \delta_{1}} \sum_{\beta, \gamma, \delta} \frac{2 V_{\alpha_{2} \beta_{2} \gamma_{2} \delta}}{\epsilon-\Xi_{\alpha_{1} \beta_{1} \alpha_{2} \beta_{2}}^{\beta \delta_{1} \gamma_{2}}}\left(\frac{V_{\alpha_{1} \beta_{1} \gamma \delta_{1}}}{\epsilon-\Xi_{\delta \alpha_{1} \beta_{1}}^{\beta \delta_{1}}}+\frac{V_{\alpha_{1} \beta_{1} \gamma \delta_{1}}}{\epsilon-\Xi_{\gamma \alpha_{2} \beta_{2}}^{\beta \gamma_{2}}}\right) \\
& \times \frac{V_{\delta \gamma \beta \alpha}}{\epsilon-\Xi_{\gamma \delta}^{\beta}} \hat{c}_{\alpha_{2}}^{\dagger} \hat{c}_{\beta_{2}}^{\dagger} \hat{c}_{\alpha_{1}}^{\dagger} \hat{c}_{\beta_{1}}^{\dagger} \hat{c}_{\beta} \hat{c}_{\delta_{1}} \hat{c}_{\gamma_{2}}\left|\Psi_{k}\right\rangle .
\end{aligned}
$$

Two terms in the second factor correspond to two ways to obtain the final seven-particle state. One immediatley notices that the matrix elements in the two terms are the same. Moreover, the denominators in two terms can be 
combined as

$$
\frac{1}{\epsilon-\Xi_{\delta \alpha_{1} \beta_{1}}^{\beta \delta_{1}}}+\frac{1}{\epsilon-\Xi_{\gamma \alpha_{2} \beta_{2}}^{\beta \gamma_{2}}}=\frac{\epsilon-\Xi_{\gamma \delta}^{\beta}+\epsilon-\Xi_{\alpha_{1} \beta_{1} \alpha_{2} \beta_{2}}^{\beta \delta_{1} \gamma_{2}}}{\left(\epsilon-\Xi_{\delta \alpha_{1} \beta_{1}}^{\beta \delta_{1}}\right)\left(\epsilon-\Xi_{\gamma \alpha_{2} \beta_{2}}^{\beta \gamma_{2}}\right)}
$$

Generally, one seeks to maximize the transition amplitude by choosing the smallest possible denominators for each of the three factors in Eq. (40) independently. Each denominator being of the order of $\delta_{\zeta} / K$, the whole expression would be proportional to $K^{3}$. However, one can see immediately from Eq. (41) that small denominators of the first and the third factors of Eq. (40) appear in the numerator of the second factor. Thus the amplitudes shown Fig. 2 (c.2)-(c.3) are not indepenedent, and in fact, cancel each other, producing a contribution proportional to $K^{2}$ instead of $K^{3}$. One can follow similar cancellations in any order of the perturbation theory. Therefore, the estimate (39) is not valid and scaling (31) remains intact. Therefore, one can use the Anderson result [1] for the critical connecitivity and obtain the estimate for the transition temperature $T_{c}$

$$
K \simeq \frac{T_{c}}{\delta_{\zeta}} \simeq \frac{1}{\lambda|\ln \lambda|}
$$

the many-body mobility edge $\mathcal{E}_{c}$ is then found from Eq. (22b). The qualitative estimate (42) is in agreement with the result of a quantitative calculation performed in subsequent sections for a specific model [Eq. (160)].

The arguments given in this section show that, even though the many-body problem (24) exhibits a close analogy with the problem of Anderson localization [1], an exact mapping to the Anderson model on some graph, like one suggested in Ref. [16], is problematic. First, the geometric structure of this graph is unknown. Analogy with the Cayley tree, popular due to the exact solvability of the corresponding Anderson model [17], is not applicable, strictly speaking, because in the many-body problem two states can be connected in more than one way, which would be prohibited for the Cayley tree. Second, the many-body problem does not allow for simple counting of statistically independent paths, which was the main idea of the Anderson's solution [1], as transition amplitudes, corresponding to seemingly different paths, exhibit striking correlations. Therefore, the appropriate starting point is the formalism where the cancellation of the factorial terms is taken into account automatically. This formalism is in fact none but the well-known diagrammatic technique for the many-body system [21], as one diagram includes the sum of all the processes obtained by trivial permutations. The correlations between different diagrams are much weaker and can be treated perturbatively. The subsequent sections are devoted to the statistical analysis for the many-body Green functions in a basis of the localized one-particle states. The transition resembling the Anderson's transition on the Cayley tree $[17,22]$ will be indeed demonstrated, despite of the subtleties discussed above. 


\section{Choice of the model}

The purpose of this section is to introduce the simplest model describing the metal-insulator transition for interacting electrons as a coarse-grained version of the initial Hamiltonian of interacting electrons in the disorder potential.

The Hamiltonian $\hat{H}$ of electrons placed in a disorder potential $U(\boldsymbol{r})$ and interacting with each other via a two-body interaction potential $V\left(\boldsymbol{r}, \boldsymbol{r}^{\prime}\right)=V\left(\boldsymbol{r}^{\prime}, \boldsymbol{r}\right)$ can be written in the basis of the exact single-electron wavefunctions ${ }^{4}$ as

$$
\begin{aligned}
& \hat{H}=\hat{H}_{0}+\hat{V}_{i n t} ; \\
& \hat{H}_{0}=\sum_{\alpha} \xi_{\alpha} \hat{c}_{\alpha}^{\dagger} \hat{c}_{\alpha} ; \\
& \hat{V}_{i n t}=\frac{1}{2} \sum_{\alpha \beta \gamma \delta} V_{\alpha \beta \gamma \delta} \hat{c}_{\alpha}^{\dagger} \hat{c}_{\beta}^{\dagger} \hat{c}_{\gamma} \hat{c}_{\delta} ; \\
& V_{\alpha \beta \gamma \delta}=\frac{1}{2} \int V\left(\boldsymbol{r}, \boldsymbol{r}^{\prime}\right) \varrho_{\alpha \delta}(\boldsymbol{r}) \varrho_{\beta \gamma}\left(\boldsymbol{r}^{\prime}\right) \mathrm{d}^{d} \boldsymbol{r} \mathrm{d}^{d} \boldsymbol{r}^{\prime} ; \\
& \varrho_{\alpha \delta}(\boldsymbol{r})=\phi_{\alpha}^{*}(\boldsymbol{r}) \phi_{\delta}(\boldsymbol{r}),
\end{aligned}
$$

where $\hat{c}_{\alpha}^{\dagger}$ and $\hat{c}_{\alpha}$ are the fermion creation and annihilation operators:

$$
\left\{\hat{c}_{\alpha}^{\dagger} ; \hat{c}_{\beta}\right\}=\delta_{\alpha \beta}, \quad\left\{\hat{c}_{\alpha} ; \hat{c}_{\beta}\right\}=\left\{\hat{c}_{\alpha}^{\dagger} ; \hat{c}_{\beta}^{\dagger}\right\}=0,
$$

and $\{\ldots ; \ldots\}$ stand for the anticommutator.

Index $\alpha$ labels the one-particle state and $\xi_{\alpha}$ is the corresponding eigenvalue:

$$
\left[-\frac{\nabla^{2}}{2 m}+U(\boldsymbol{r})-\epsilon_{F}\right] \phi_{\alpha}(\boldsymbol{r})=\xi_{\alpha} \phi_{\alpha}(\boldsymbol{r}) .
$$

To characterize the single-particle spectrum, we introduce average density of states (DoS) per unit volume

$$
\nu=\frac{1}{\mathcal{V}} \sum_{\alpha}\left\langle\delta\left(\xi_{\alpha}\right)\right\rangle
$$

where $\langle\ldots\rangle$ denote the averaging over the disorder realization and $\mathcal{V}$ is the volume of $d$-dimensional system.

Both one-particle energies and interaction matrix elements are random quantities which are functionals of the random potential $U(\boldsymbol{r})$. The usual pro-

4 For simplicity, we consider the electrons as the spinless fermions. There is no reason to believe that the straightforward inclusion of the spin degrees of freedom affects qualitatively the final conclusions. 
gram [15] of averaging over the disorder realizations faces difficulty when the relevant spatial scale becomes comparable with the one-particle localization length $\zeta_{l o c}$. However, we would like to discuss the physics associated with length scale much larger than $\zeta_{l o c}$. To perform this task, we adopt the reduced statistical model for the matrix elements and eigenfunctions which, however, keeps all essential physics intact. We will not discuss how the parameters of this low-energy effective model are connected to the properties of the system in the high-temperature regime [15].

To write down this model, we use the following properties of the localized non-interacting system [23]:

(i) The typical wavefunctions have exponential envelopes,

$$
-\ln \left|\phi_{\alpha}(\boldsymbol{r})\right|=\frac{\left|\boldsymbol{r}-\boldsymbol{\rho}_{\alpha}\right|}{\zeta_{l o c}},
$$

where $\zeta_{l o c}$ is the localization length, and $\boldsymbol{\rho}_{\alpha}$ characterizes the position of the "center of mass" of the wavefunction. ${ }^{5}$

(ii) Levels $\xi_{\alpha}, \xi_{\beta}$ repel each other for $\left|\xi_{\alpha}-\xi_{\beta}\right| \lesssim \delta_{\zeta} \exp \left[-\frac{\left|\boldsymbol{\rho}_{\beta}-\boldsymbol{\rho}_{\alpha}\right|}{\zeta_{\text {loc }}}\right]$, and are almost independent otherwise. Here we introduce the main energy scale of the problem:

$$
\delta_{\zeta} \equiv \frac{1}{\nu \zeta_{\text {loc }}^{d}}
$$

which has the meaning of one-particle level spacing on the localization length ( $d$ is the dimensionality of the system);

(iii) The overlap between wavefunctions decays exponentially with the distance, see Eq. (45), whereas the overlap between the wavefunctions with the centers of mass at the distance much smaller than the localization length strongly depends on the corresponding one-particle energies $\xi_{\alpha}$. In particular, for the estimate of the contribution to the interaction matrix elements (43d) from the distances smaller than the localization length one can approximate functions $\varrho(\boldsymbol{r})$ from Eq. (43e) as Gaussian variables with the correlation function ${ }^{6}$

$$
\left\langle\varrho_{\alpha \beta}\left(\boldsymbol{r}_{1}\right) \varrho_{\alpha \beta}^{*}\left(\boldsymbol{r}_{2}\right)\right\rangle \simeq \frac{\delta_{\zeta} \Pi_{d}\left(\frac{\left|\boldsymbol{r}_{1}-\boldsymbol{r}_{2}\right|}{L_{\omega_{\alpha \beta}}}\right)}{\omega_{\alpha \beta} \zeta_{l o c}^{d} L_{\omega_{\alpha \beta}}^{d}}
$$

5 Everywhere we assume that at the distances smaller than the localization length $\zeta_{l o c}$ the wave functions are well described by the semiclassical approximation, i.e., $\left(m \epsilon_{F}\right)^{1 / 2} \zeta_{l o c} \gg 1$. We will also neglect the weak energy dependence of the localization length as well as of the average DoS in Eq. (44).

6 We will neglect the correlation of the wavefunctions stemming from the Cooperon contributions, i.e., assume the unitary ensemble 
where $\omega_{\alpha \beta} \equiv\left|\xi_{\alpha}-\xi_{\beta}\right|$, diffusion length is given by $L_{\omega}=(D / \omega)^{1 / 2}$, and $D$ is the classical diffusion coefficient. Dimensionless diffuson $\Pi_{d}(x)$ is given by

$$
\Pi_{d}(x) \equiv \operatorname{Re} \int \frac{\mathrm{d}^{d} \boldsymbol{Q}}{(2 \pi)^{d}} \frac{\mathrm{e}^{i Q_{x} x}}{i+Q^{2}},
$$

and it decays exponentially at $x \gg 1$. Equation (47) is valid provided $\left|\boldsymbol{r}_{1,2}-\boldsymbol{\rho}_{\alpha, \beta}\right| \ll \zeta_{\text {loc }}$ so that the results obtained for the metallic states are applicable ${ }^{7}$. It is important to emphasize that Eq. (47) implies the relation between the spatial distance and the energy transfer and does not impose any restrictions on the values of energy themselves.

These facts suggest the following coarse-grained version of the Hamiltonian (43). We discretize the space into a $d$-dimensional cubic lattice with the lattice constant $\zeta_{l o c}$, the coordinate of each site will be labeled as $\boldsymbol{\rho}$. We will call the unit cell of this lattice a localization cell. Each localization cell, $\boldsymbol{\rho}$, contains large number of levels, $N \rightarrow \infty$, labeled by integer $l$,

$$
-\frac{N \delta_{\zeta}}{2}<\xi_{l}(\boldsymbol{\rho})<\frac{N \delta_{\zeta}}{2} ; \quad 1 \leq l \leq N .
$$

Two levels $\xi_{l}\left(\boldsymbol{\rho}_{1}\right), \xi_{m}\left(\boldsymbol{\rho}_{2}\right)$ are independent for $\boldsymbol{\rho}_{1} \neq \boldsymbol{\rho}_{2}$ and repel each other otherwise. To characterize this repulsion, let us consider the probability, $P(n, E)$, to find $n$ levels in the energy interval of the width $E \gg \delta_{\zeta}$. We will write this probability in the form

$$
P(n, E)=\left[\frac{\mathrm{e}^{-E / \delta_{\zeta}}}{n !}\left(\frac{E}{\delta_{\zeta}}\right)^{n}\right] \exp \left[-\mathcal{P}\left(\frac{n \delta_{\zeta}}{E}\right)\right] .
$$

The first factor in this expression characterizes the Poisson distribution of the independent random levels. The last exponential describes the level repulsion. The precise functional form of this repulsion is not important for us, we require only

$$
\lim _{x \rightarrow \infty} x^{-1} \mathcal{P}(x)=\infty,
$$

which is a natural assumption for any repelling levels with the scale of the repulsion determined by $\delta_{\zeta}$.

As follows from previous discussion of the structure of the wave functions, the interaction matrix elements are largest for the states belonging to one localization cell, and decay exponentially with the distance. Thus, we will take into account only interaction within one localization cell.

The interaction within one cell, however, can not cause the delocalization in space and, in fact, does not cause any qualitative effects at all. Therefore, we

$\overline{7 \text { For } d} \geq 3$ Anderson model with all the one-particle states localized the wavefunctions at distances smaller than $\zeta_{l o c}$ are critical rather than metallic. It does not affect the final form of the effective model proposed in this section. 
will take into account the single-electron hopping from one localization cell $\boldsymbol{\rho}_{1}$ to its nearest neighbour $\boldsymbol{\rho}_{2}$. Being small, this hopping does not change the localization properties of the single electron wave function, however, taken together with the electron-electron interaction would eventually lead to the transiton.

The resulting Hamiltonian, thus, takes the form

$$
\begin{aligned}
& \hat{H}=\hat{H}_{0}+\hat{V}_{i n t} ; \\
& \hat{H}_{0}=\sum_{\boldsymbol{\rho}, l} \hat{c}_{l}^{\dagger}(\boldsymbol{\rho})\left[\xi_{l}(\boldsymbol{\rho}) \hat{c}_{l}(\boldsymbol{\rho})+I \delta_{\xi} \sum_{\boldsymbol{a}, m} \hat{c}_{m}(\boldsymbol{\rho}+\boldsymbol{a})\right] . \\
& \hat{V}_{i n t}=\frac{1}{2} \sum_{l_{1} l_{2} j_{1} j_{2} ; \boldsymbol{\rho}} V_{l_{1} l_{2}}^{j_{1} j_{2}}(\boldsymbol{\rho}) \hat{c}_{l_{1}}^{\dagger}(\boldsymbol{\rho}) \hat{c}_{l_{2}}^{\dagger}(\boldsymbol{\rho}) \hat{c}_{j_{2}}(\boldsymbol{\rho}) \hat{c}_{j_{1}}(\boldsymbol{\rho}),
\end{aligned}
$$

where $\left\{\hat{c}_{i}^{\dagger}\left(\boldsymbol{\rho}_{1}\right) ; \hat{c}_{j}\left(\boldsymbol{\rho}_{2}\right)\right\}=\delta_{i j} \delta_{\boldsymbol{\rho}_{1} \boldsymbol{\rho}_{2}},\left\{\hat{c}_{i}\left(\boldsymbol{\rho}_{1}\right) ; \hat{c}_{j}\left(\boldsymbol{\rho}_{2}\right)\right\}=\left\{\hat{c}_{i}^{\dagger}\left(\boldsymbol{\rho}_{1}\right) ; \hat{c}_{j}^{\dagger}\left(\boldsymbol{\rho}_{2}\right)\right\}=0$.

Here $\boldsymbol{a}$ are the vectors connecting the cell $\boldsymbol{\rho}$ to its nearest neighbors. Dimensionless hopping parameter, $I$, such that

$$
I \ll \frac{1}{2 d \ln 2 d},
$$

is introduced to control further perturbative expansion. We will chose $I>0$ : this choice does not affect any conclusions, as $I$ will connect terms with the random signs.

The antisymmetrized coefficients $V_{l_{1} l_{2}}^{j_{1} j_{2}}(\boldsymbol{\rho})=V_{l_{2} l_{1}}^{j_{2} j_{1}}(\boldsymbol{\rho})=-V_{l_{1} l_{2}}^{j_{2} j_{1}}(\boldsymbol{\rho})$ are random numbers. Because the physical processes discussed in this paper are associated with the counting of the resonant denominators, the particular choice of the statisitical distribution of $V_{l_{1} l_{2}}^{j_{1} j_{2}}(\boldsymbol{\rho})$ is not really important. For the calculational convenience we choose the binary distribution

$$
V_{l_{1} l_{2}}^{j_{1} j_{2}}=\frac{\lambda \delta_{\zeta} \sigma_{l_{1}}^{j_{1}} \sigma_{l_{2}}^{j_{2}}}{2} \Upsilon\left(\frac{\omega_{j_{1} l_{1}}}{\delta_{\zeta}}\right) \Upsilon\left(\frac{\omega_{j_{2} l_{2}}}{\delta_{\zeta}}\right)-\left(l_{1} \leftrightarrow l_{2}\right)
$$

where $\lambda \simeq I \ll 1$ is a dimensionless parameter allowing to control the perturbative expansion, $\omega_{l j}=\xi_{l}-\xi_{j}$ and we omitted argument $\boldsymbol{\rho}$ on both sides of the equation. We chose $\lambda>0$ without loss of the generality, as all the effects which will be considered are not sensitive to the sign of $\lambda$. Function $\Upsilon(x)$ is introduced to describe the interaction decaying rapidly with the distance between the levels in the energy space, see Eq. (47). As the smallest linear scale in the reduced model is $\zeta_{l o c}$ the maximal energy transfer which is permissible 
to consider in the model is of the order of $\delta_{\zeta}$. Thus, we choose ${ }^{8}$

$$
\Upsilon(x)=\theta\left(\frac{M}{2}-|x|\right) ; \quad 1 \ll M \lesssim \frac{1}{\sqrt{\lambda}}
$$

Actual value of the parameter $M$ is not well defined and we will consider it as the initial data for the effective Hamiltonian. The significance of the upper bound to $M$ for the consistency of the theory will be clear later, see discussion after Eq. (80) as well as Sec. 7.1.

The signs for different wave functions are not correlated, so

$$
\left[\sigma_{l}^{j}(\boldsymbol{\rho})\right]^{2}=1 ; \quad\left\langle\sigma_{l}^{j}(\boldsymbol{\rho}) \sigma_{l^{\prime}}^{j^{\prime}}\left(\boldsymbol{\rho}^{\prime}\right)\right\rangle=\delta_{\boldsymbol{\rho}, \boldsymbol{\rho}^{\prime}} \delta_{l l^{\prime}} \delta_{j j^{\prime}}
$$

Equations (48) constitute a complete formulation of the reduced model for the interacting electrons in system with localized one-particle states. This model will be analyzed in the subsequent sections to show the stability of both hightemperature phase (metal) and low-temperature phase (insulator).

\section{Formalism.}

The purpose of this section is to describe the machinery which enables us to put the previous qualitative arguments into the context of the usual manybody theory of non-equlibrium systems. We will start with the outline of the Keldysh formalism for the exact (non-averaged) Green functions corresponding to Hamiltonian (48) in Sec. 4.1 and formulate which quantity describes the metal-insulator transition in Sec. 4.2. Next, in Sec. 4.3 we will describe our main working approximation which corresponds to the summation of all the rainbow diagrams (SCBA). The justification of the validity of this approximation for the description of the transition is postponed until Sec. 7.

\subsection{Time evolution equations and basic definitions.}

We intend to describe both metallic and insulating regimes. In the latter regime relaxation dynamics is absent, there is no mechanism to establish the thermal equilibrium, and the temperature itself is not defined. Therefore, the

8 It can be shown that taking into account the algebraic decay of $\Upsilon(x), x \gg 1$ would require the consideration of the spatial correlation of the wave functions, so the approximation for the interaction matrix elements to be independent of each other and of the hopping would be false even on a qualitative level. 
only appropriate formal framework is the non-equilibrium (Keldysh) formalism [24]. We define the corresponding Green functions as

$$
\begin{aligned}
& \mathcal{G}_{l}^{R}\left(t_{1}, t_{2} ; \boldsymbol{\rho}\right)=-i \theta\left(t_{1}-t_{2}\right)\left\langle\left\langle\left\{\hat{c}_{l}\left(t_{1}, \boldsymbol{\rho}\right) ; \hat{c}_{l}^{\dagger}\left(t_{2}, \boldsymbol{\rho}\right)\right\}\right\rangle\right\rangle, \\
& \mathcal{G}_{l}^{A}\left(t_{1}, t_{2} ; \boldsymbol{\rho}\right)=i \theta\left(t_{2}-t_{1}\right)\left\langle\left\langle\left\{\hat{c}_{l}\left(t_{1}, \boldsymbol{\rho}\right) ; \hat{c}_{l}^{\dagger}\left(t_{2}, \boldsymbol{\rho}\right)\right\}\right\rangle\right\rangle, \\
& \mathcal{G}_{l}^{K}\left(t_{1}, t_{2} ; \boldsymbol{\rho}\right)=-i\left\langle\left\langle\left[\hat{c}_{l}\left(t_{1}, \boldsymbol{\rho}\right) ; \hat{c}_{l}^{\dagger}\left(t_{2}, \boldsymbol{\rho}\right)\right]\right\rangle\right\rangle,
\end{aligned}
$$

where $\theta(t)$ is the Heaviside step function, and the fermionic operators are written in the Heisenberg representation. Quantum mechanical averaging $\langle\langle. .\rangle$. is performed over an arbitrary density matrix to be found from the solution of the kinetic equation. To avoid misunderstanding, we emphasize that no averaging over the disorder realization is assumed in Eq. (49).

We parametrize the Keldysh Green function as

$$
\mathcal{G}_{l}^{K}(\boldsymbol{\rho})=\mathcal{G}_{l}^{R}(\boldsymbol{\rho}) \circ n_{l}(\boldsymbol{\rho})-n_{l}(\boldsymbol{\rho}) \circ \mathcal{G}_{l}^{A}(\boldsymbol{\rho})
$$

where we omitted the time arguments for brevity and introduced the shorthand notation

$$
\mathcal{C} \circ \mathcal{D} \equiv \int \mathrm{d} t_{3} \mathcal{C}\left(t_{1}, t_{3}\right) \mathcal{D}\left(t_{3}, t_{2}\right)
$$

for arbitrary functions $\mathcal{C}, \mathcal{D}$.

In the thermodynamic equilibrium

$$
n_{l}(\boldsymbol{\rho} ; \epsilon, t)=1-2 f_{F}(\epsilon)
$$

where $f_{F}(\epsilon)$ is the Fermi distribution function with arbitray temperature and chemical potential, and the time Wigner transform is defined as usual:

$$
\mathcal{D}\left(t+\frac{\tau}{2}, t-\frac{\tau}{2}\right)=\int \frac{\mathrm{d} \epsilon}{2 \pi} \mathrm{e}^{-i \epsilon \tau} \mathcal{D}(\epsilon, t)
$$

In the absence of interaction $n_{l}\left(\boldsymbol{\rho} ; \epsilon=\xi_{l}(\boldsymbol{\rho}, t)\right)$ characterizes the occupation of the level $n_{l}\left(\boldsymbol{\rho} ; \xi_{l}(\boldsymbol{\rho}), t\right)=1(-1)$ for an empty (filled) level $(\boldsymbol{\rho}, l)$.

In what follows we will use standard diagrammatic technique for the perturbative expansion for the model (48). The basic elements of this technique are defined in Fig. 3.

The Green functions (49) corresponding to the Hamiltonian (48) satisfy the 
a)

$$
\frac{(l, \boldsymbol{\rho})}{\mu_{1}}=i\left[\hat{G}_{l}(\boldsymbol{\rho})\right]_{\mu_{1} \mu_{2}}=i\left[\begin{array}{cc}
G_{l}^{R}(\boldsymbol{\rho}) & G_{l}^{K}(\boldsymbol{\rho}) \\
0 & G_{l}^{A}(\boldsymbol{\rho})
\end{array}\right]_{\mu_{1} \mu_{2}}
$$

\begin{tabular}{ll}
$(l, \boldsymbol{\rho})$ & $G_{l}^{R}(\boldsymbol{\rho})=\left[G_{l}^{A}(\boldsymbol{\rho})\right]^{*}=\frac{1}{\epsilon-\xi_{l}(\boldsymbol{\rho})+i 0^{+}}$ \\
\hline & $G_{l}^{K}(\boldsymbol{\rho})=-2 \pi i n_{l}(\boldsymbol{\rho}, \epsilon) \delta\left[\epsilon-\xi_{l}(\boldsymbol{\rho})\right]$
\end{tabular}

b)

c)

$\underset{\mu_{1}}{(l, \boldsymbol{\rho})}=i\left[\hat{\mathcal{G}}_{l}(\boldsymbol{\rho})\right]_{\mu_{1} \mu_{2}} ; \quad \mu_{1} \underline{\mu}_{\mu_{2}}=-i I \delta_{\xi}\left[\hat{\tau}^{0}\right]_{\mu_{1} \mu_{2}}\left(\boldsymbol{\rho}_{1}, \boldsymbol{\rho}_{2}\right)_{n n} ;$

d)

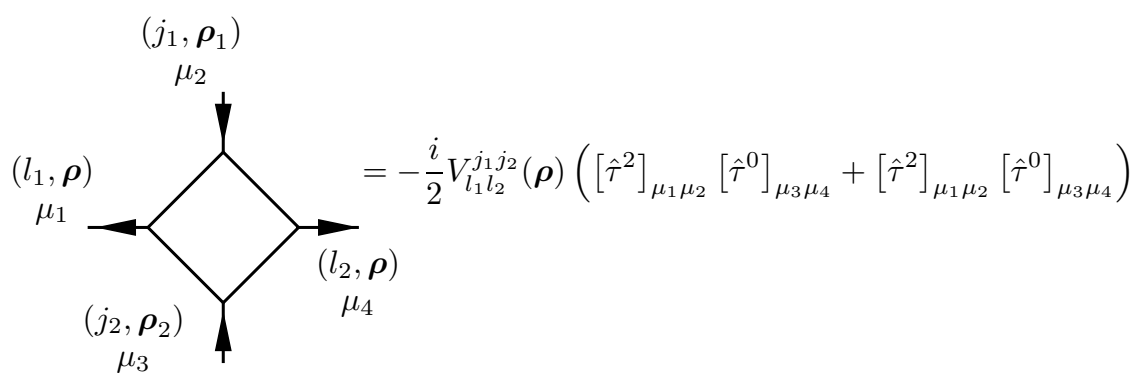

e)

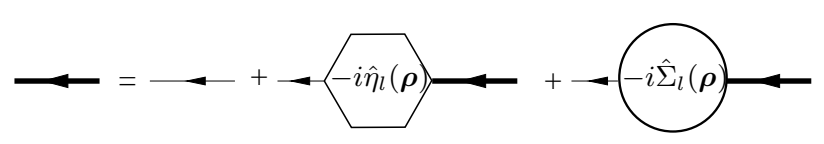

$-i \hat{\Sigma}_{l}(\boldsymbol{\rho})=$ Fig. $4+$ Fig. $5+\ldots$

Fig. 3. (a-d) Basic elements of the diagrammatic technique. The Keldysh space is labeled by $\mu_{i}$, and the Pauli matrices in Keldysh space are defined in Eq. (54). Line crossing the Green function excludes the orbital $(l, \boldsymbol{\rho})$ from the summation. (e) Representation of Eqs. (54) - (55).

equations (we omitted time arguments for brevity)

$$
\begin{aligned}
& {\left[i \partial_{t_{1}}-\xi_{l}(\boldsymbol{\rho})\right] \hat{\mathcal{G}}_{l}(\boldsymbol{\rho})=\hat{\tau}_{0} \delta\left(t_{1}-t_{2}\right)+\hat{\boldsymbol{\Sigma}}_{l}(\boldsymbol{\rho}) \circ \hat{\mathcal{G}}_{l}(\boldsymbol{\rho}) ;} \\
& {\left[-i \partial_{t_{2}}-\xi_{l}(\boldsymbol{\rho})\right] \hat{\mathcal{G}}_{l}(\boldsymbol{\rho})=\hat{\tau}_{0} \delta\left(t_{1}-t_{2}\right)+\hat{\mathcal{G}}_{l}(\boldsymbol{\rho}) \circ \hat{\boldsymbol{\Sigma}}_{l}(\boldsymbol{\rho}) ;} \\
& \hat{\mathcal{G}}=\left[\begin{array}{cc}
\mathcal{G}_{l}^{R}(\boldsymbol{\rho}) & \mathcal{G}_{l}^{K}(\boldsymbol{\rho}) \\
0 & \mathcal{G}_{l}^{A}(\boldsymbol{\rho})
\end{array}\right]_{K} ; \hat{\boldsymbol{\Sigma}}=\left[\begin{array}{rr}
\boldsymbol{\Sigma}_{l}^{R}(\boldsymbol{\rho}) & \boldsymbol{\Sigma}_{l}^{K}(\boldsymbol{\rho}) \\
0 & \boldsymbol{\Sigma}_{l}^{A}(\boldsymbol{\rho})
\end{array}\right]_{K} \\
& \hat{\tau}^{0}=\left[\begin{array}{ll}
1 & 0 \\
0 & 1
\end{array}\right]_{K}^{;} \quad \hat{\tau}^{2}=\left[\begin{array}{ll}
0 & 1 \\
1 & 0
\end{array}\right]_{K}
\end{aligned}
$$


a)

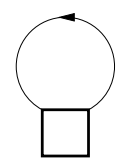

b)

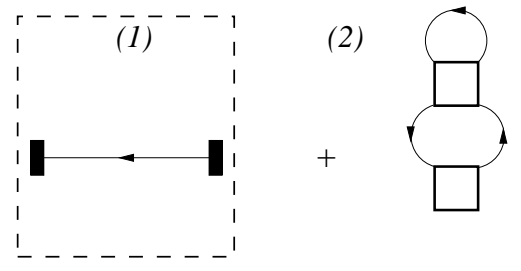

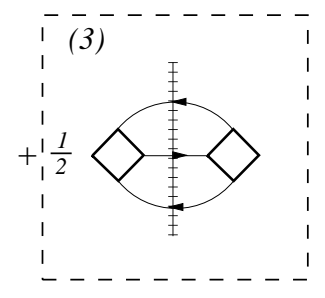

(3)

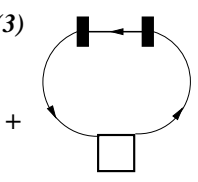

c)

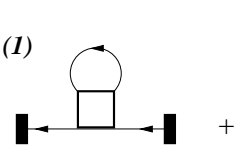

(2)
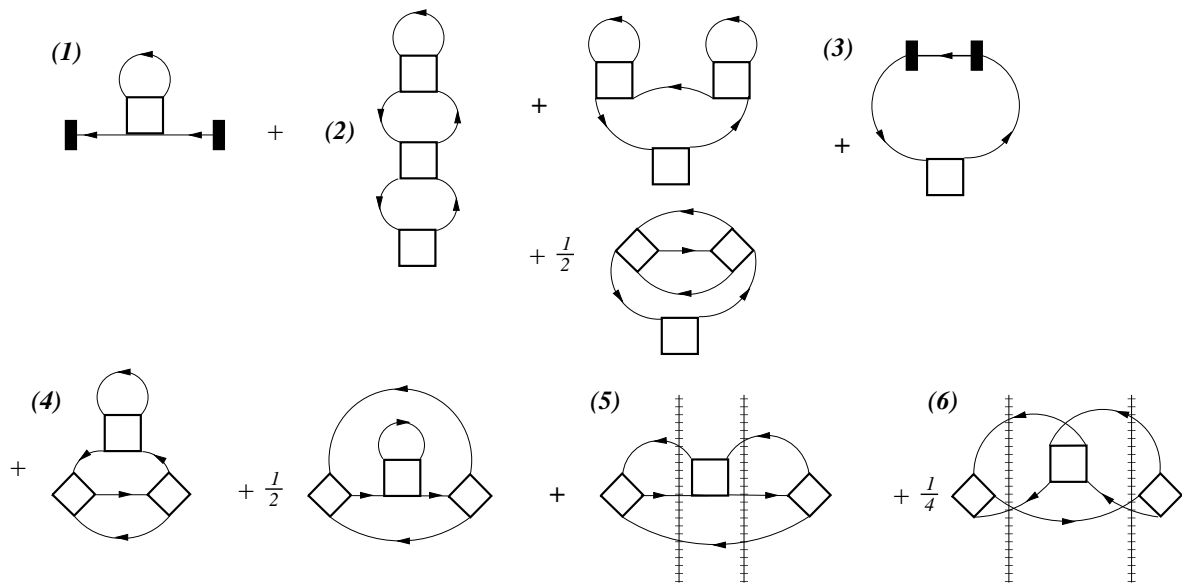

Fig. 4. First (a), second (b) and third (c) orders contributions to the self-energy $\hat{\Sigma}_{l}(\boldsymbol{\rho})$. The diagrams which will be taken into account in the self-consistent Born approximation are highlighted by the dashed frames. Cross-sections of the diagram which produce the imaginary part of the self-energy are denoted by the dotted lines.

The self-energy $\hat{\boldsymbol{\Sigma}}_{l}(\boldsymbol{\rho})$ is given by

$$
\hat{\boldsymbol{\Sigma}}_{l}(\boldsymbol{\rho})=\hat{\eta}+\hat{\Sigma}_{l}(\boldsymbol{\rho}),
$$

where $\hat{\eta}$ originates from the coupling of the sysstem to an external bath with regular continuous spectrum (it will be discussed in more details later). This coupling has to be kept small but finite and can be put to zero only in the end of the calculation. Self-enery $\hat{\Sigma}_{l}(\boldsymbol{\rho})$ originates from the electron-electron interaction and hopping and represents the sum of all diagrams which cannot be separated by cutting one-electron line $(l, \boldsymbol{\rho})$, see Figs. 4,5 .

Substitution of Eq. (50) into the Keldysh component of Eq. (54) yields

$$
\left(\partial_{t_{1}}+\partial_{t_{2}}\right) n_{l}(\boldsymbol{\rho})=-i \boldsymbol{\Sigma}_{l}^{R}(\boldsymbol{\rho}) \circ n_{l}(\boldsymbol{\rho})+i n_{l}(\boldsymbol{\rho}) \circ \boldsymbol{\Sigma}_{l}^{A}(\boldsymbol{\rho})+i \boldsymbol{\Sigma}_{l}^{K}(\boldsymbol{\rho}) .
$$

On the next step we restrict ourselves to the consideration of very slow dynamics. In this case one can perform the time Wigner transform (53) in Eq. (56) and obtain the quantum Boltzmann equation

$$
\partial_{t} n_{l}(\epsilon ; \boldsymbol{\rho}, t)+\left[\delta \xi_{l}(\epsilon ; \boldsymbol{\rho} ;\{n\}) \stackrel{*}{,} n_{l}(\boldsymbol{\rho}, \epsilon, t)\right]=\mathrm{St}_{l}\left(\epsilon ; \boldsymbol{\rho} ;\{n\},\left\{\partial_{t} n\right\}\right)+\mathrm{St}_{l}^{b a t h}
$$


(2)

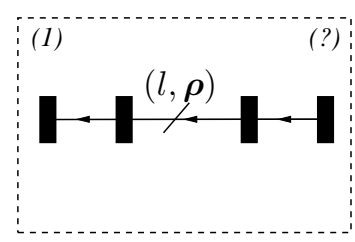

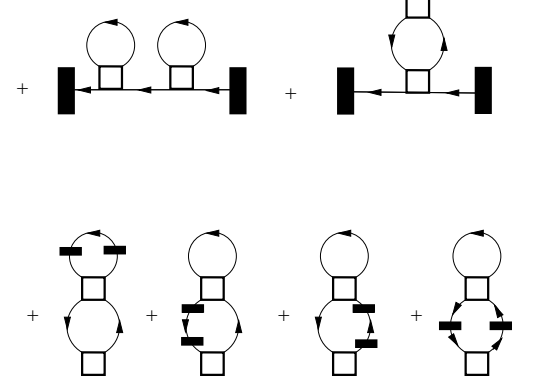

(3)

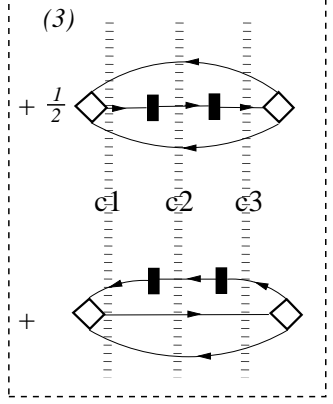

(3)
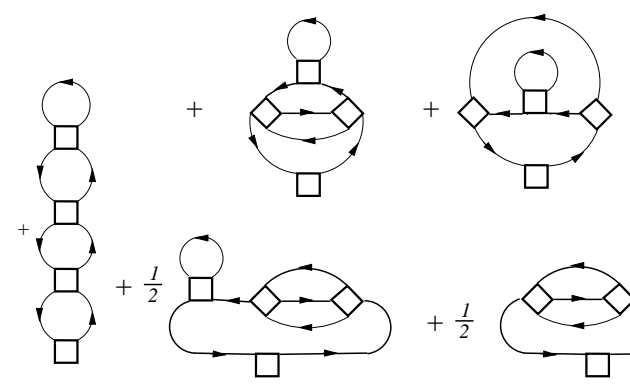

$+\frac{1}{2}$
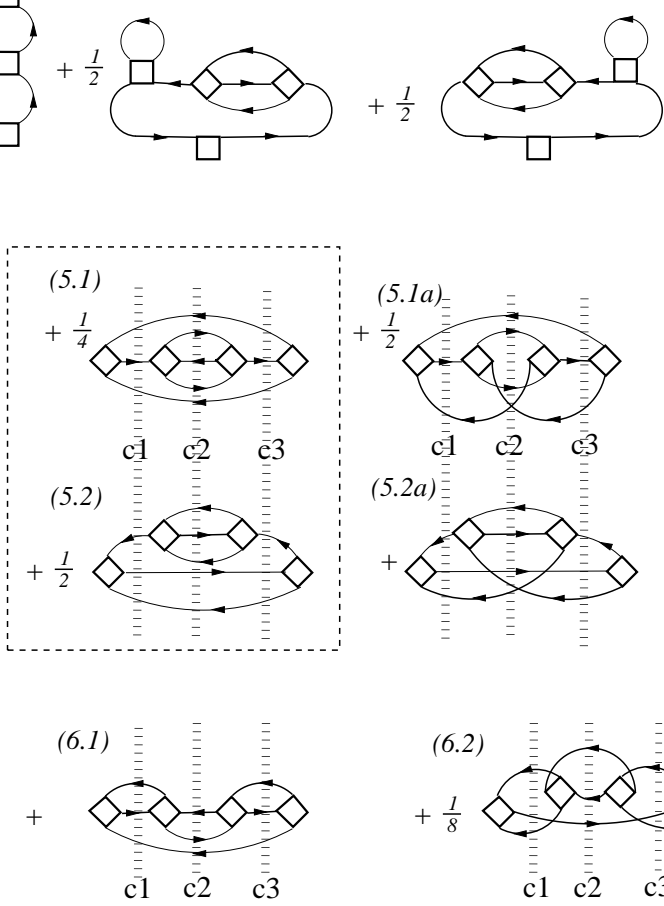
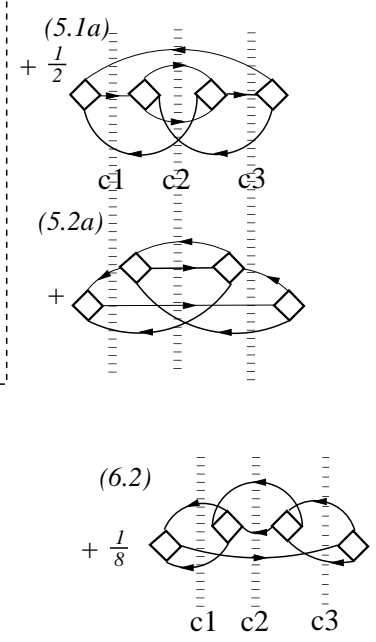

(4)
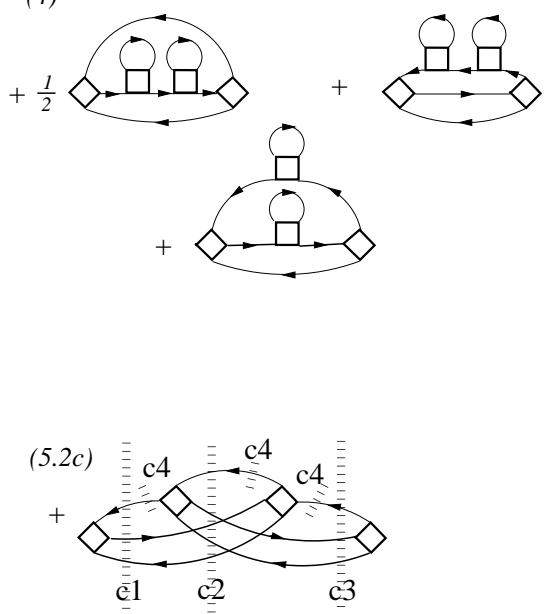

$(5.2 d)$

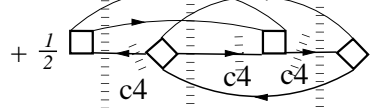

$+\frac{1}{2}$

Fig. 5. Fourth-order contributions to the self-energy $\hat{\Sigma}_{l}(\boldsymbol{\rho})$. The diagrams which will be taken into account in the self-consistent Born approximation are highlighted by the dashed frames. Cross-sections of the diagram which produce the imaginary part of the self-energy are denoted by the dotted lines and denoted by $\mathrm{c} 1-\mathrm{c} 4$. The crosssection c4 implies cutting three lines at the same time.

where the collision integrals are defined as

$$
\begin{aligned}
& \mathrm{St}_{l}\left(\epsilon ; \boldsymbol{\rho} ;\{n\},\left\{\partial_{t} n\right\}\right)=-2 \Gamma_{l}\left(\epsilon ; \boldsymbol{\rho} ;\{n\},\left\{\partial_{t} n\right\}\right) n_{l}(\boldsymbol{\rho}, \epsilon, t)+i \Sigma_{l}^{K}\left(\boldsymbol{\rho} ; \epsilon ;\{n\},\left\{\partial_{t} n\right\}\right) ; \\
& \mathrm{St}_{l}^{\text {bath }}=-2 \operatorname{Im} \eta^{A} n_{l}(\boldsymbol{\rho}, \epsilon, t)+i \eta^{K} .
\end{aligned}
$$


Hereinafter, the time Poisson brackets are defined as

$$
[\mathcal{C} * \mathcal{D}] \equiv \partial_{t} \mathcal{C} \partial_{\epsilon} \mathcal{D}-\partial_{\epsilon} \mathcal{C} \partial_{t} \mathcal{D}
$$

for arbitrary functions $\mathcal{C}(\epsilon, t), \mathcal{D}(\epsilon, t)$. The entries in Eq. (57) are defined by

$$
\Sigma^{R}=\delta \xi-i \Gamma ; \quad \Sigma^{A}=\delta \xi+i \Gamma ;
$$

where we suppressed all arguments which are the same as in Eq. (58). Using Eqs. (60) and the analytic properties of the retarded and advanced Green functions one obtains

$$
\begin{aligned}
\mathcal{G}_{l}^{R}(\boldsymbol{\rho} ; \epsilon, t) & =\left[\mathcal{G}_{l}^{A}(\boldsymbol{\rho} ; \epsilon, t)\right]^{*}=\int \frac{\mathrm{d} \epsilon^{\prime} A_{l}\left(\boldsymbol{\rho} ; \epsilon^{\prime}, t\right)}{\epsilon-\epsilon^{\prime}+i 0^{+}}, \\
A_{l}(\boldsymbol{\rho} ; \epsilon, t) & =\frac{\pi^{-1} \boldsymbol{\Gamma}_{l}\left(\epsilon ; \boldsymbol{\rho} ;\{n\},\left\{\partial_{t} n\right\}\right)}{\left[\epsilon-\xi_{l}(\boldsymbol{\rho})-\delta \xi(\epsilon ; \boldsymbol{\rho} ;\{n\})\right]^{2}+\left[\boldsymbol{\Gamma}_{l}\left(\epsilon ; \boldsymbol{\rho} ;\{n\},\left\{\partial_{t} n\right\}\right)\right]^{2}},
\end{aligned}
$$

and $\Gamma_{l}=\Gamma_{l}+\operatorname{Im} \eta_{l}^{A}$. Notation $\mathcal{F}(\{n\})$ means that $\mathcal{F}$ is a functional depending upon all the functions $n_{l}(\epsilon, \boldsymbol{\rho})$ but local in time. The latter functions enter the expressions through the quasistationary version of Eq. (50):

$\mathcal{G}_{l}^{K}(\boldsymbol{\rho} ; \epsilon, t)=-2 \pi i n_{l}(\boldsymbol{\rho} ; \epsilon, t) A_{l}\left(\boldsymbol{\rho} ; \epsilon^{\prime}, t\right)-i \int \mathrm{d} \epsilon^{\prime}\left[A_{l}\left(\boldsymbol{\rho} ; \epsilon^{\prime}, t\right) \stackrel{*}{*} n_{l}(\boldsymbol{\rho} ; \epsilon, t)\right] P \frac{1}{\epsilon-\epsilon^{\prime}}$,

where $P$ denotes the principal value.

What remains now, is to specify the thermal bath. As we already mentioned, the particular form of this choice is not important. We will require only that it preserves the number of particles

$$
\int \mathrm{d} \epsilon A_{l}(\boldsymbol{\rho} ; \epsilon, t) \mathrm{St}^{\text {bath }}(\epsilon, t)=0,
$$

it is local, and the collision integral $\mathrm{St}^{\text {bath }}$ is nullified by the equlibrium distribution function (52). It is easy to check that the choice

$$
\begin{aligned}
2 \operatorname{Im} \eta^{A} & =\int \mathrm{d} \omega \omega b(\omega) A_{l}(\boldsymbol{\rho} ; \epsilon-\omega, t)\left[\operatorname{coth} \frac{\omega}{2 T}+n_{l}(\boldsymbol{\rho} ; \epsilon-\omega, t)\right] \\
-i \eta^{K} & =\int \mathrm{d} \omega \omega b(\omega) A_{l}(\boldsymbol{\rho} ; \epsilon-\omega, t)\left[\operatorname{coth} \frac{\omega}{2 T} n_{l}(\boldsymbol{\rho} ; \epsilon-\omega, t)+1\right],
\end{aligned}
$$

where $b(\omega)=b(-\omega)>0$, saisfies both those requirements ${ }^{9}$.

$\overline{9}$ The subsequent formulas for non-zero coupling $\hat{\eta}$ actually may be used to describe the effect of the short-rangle electron-electron interaction on the phonon-assisted nearest neighbors hopping. 


\subsection{Formulation of the problem.}

Having introduced the definitions, we are ready to reformulate the criterion distinguishing insulating and metallic states. ${ }^{10}$ The left-hand side of the Boltzmann equation (57) describes the evolution of the occupation of the levels in a self-consistent field created by all the other electrons. This evolution is deterministic and time reversible. It is the right-hand side of the kinetic equation (collision integral) that makes the time evolution probabilistic and specifies the direction of the time arrow. Thus, the energy dependence of the decay rate $2 \Gamma_{l}(\epsilon)$ determines whether or not the irreversible evolution occurs in the system. If coupling with the environment, $b(\omega)$ from Eq. (63), is finite, $\Gamma_{l}(\epsilon)$ is positive for any energies. However, if this coupling tends to zero (but not faster than the exponential function of the volume of the system $\left.\mathcal{V}, b>\exp \left[-\mathcal{V} / \mathcal{V}_{0}\right]\right)$, two situations are possible, see Fig. 6a,b.

(i) Despite $b(\omega)$ tending to zero the number of the intermediate states via which the excitation can decay goes to infinity. This results in $\Gamma_{l}(\epsilon)$ being a smooth function of energy even at $b(\omega) \rightarrow 0$. This situation corresponds to the applicability of the Fermi golden rule, the thermal equilibrium within the system established at times independent of the external bath, so it is natural to classify this regime as metallic.

(ii) The number of the intermediate states via which the excitation can decay remains finite and independent on $b(\omega)$ as $b(\omega) \rightarrow 0$. This results in $\Gamma_{l}(\epsilon)$ to be a sequence of resonant peaks positioned at the energies of the exact excitations of the many-body system. In this case $n(\epsilon)$ will remain extremely singular function whose relaxation rate is determined by $b(\omega)$. At $b(\omega) \rightarrow 0$ the thermal equilibrium can never be reached and this regime is insulating.

Since these two behaviors are qualitatively different, there could be no smooth crossover between them, and only phase transition is possible. Therefore, to show the existence of the transition, it is sufficient to formulate the conditions at which either metallic (i) or insulating (ii) regimes are stable. It will be done in Secs. 5 and 6. The investigation of the behavior of the kinetic coefficients near the phase transition point itself will be the subject of a separate paper.

Next question one has to ask is how to distinguish between metallic and insulating phases within a statistical framework. It is clear that the positions of the peaks in $\Gamma(\epsilon)$ for the insulating regime deviate randomly with the variation of random energies $\xi_{\alpha}$ from Eq. (48). Therefore, the averaged value of the decay rate $\langle\Gamma(\epsilon)\rangle$ is qualitatively similar in both phases and can not be used for the distinction. The magnitude of the fluctuations $\left\langle[\delta \Gamma(\epsilon)]^{2}\right\rangle$ is, however, qualita-

\footnotetext{
${ }^{10}$ Subsequent discussion is a straightforward generalization of the Anderson's argument [1] to a many-body system.
} 


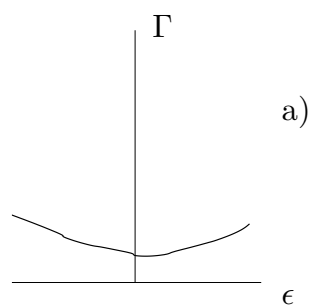

a)
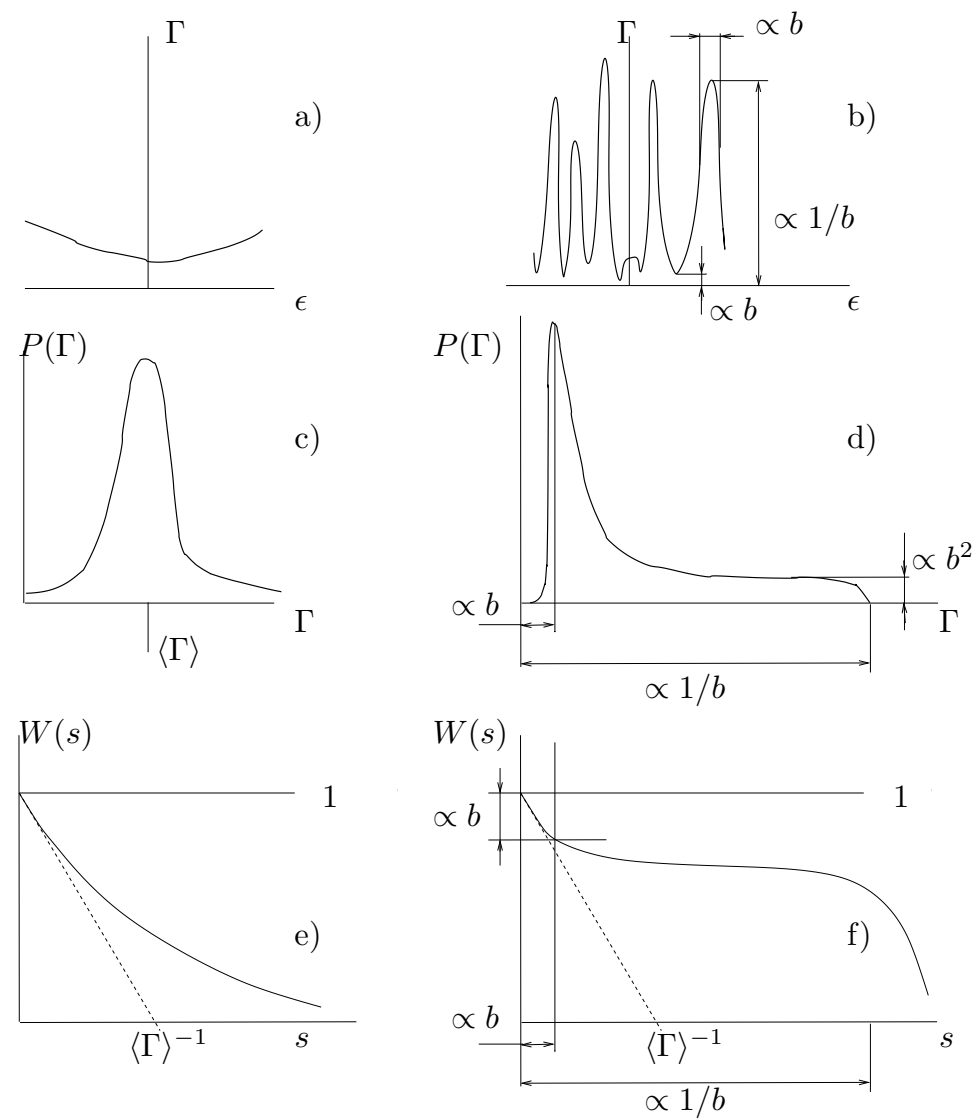

Fig. 6. Schematic energy dependence of the quasiparticle decay rate $\Gamma(\epsilon)$ for (a) metallic and (b) insulating phases. The corresponding distribution functions are sketched on panels (c), (d). The characteristic functions are plotted on panels (e), (f).

tively different in both cases. In the metallic case the averaging is performed with respect to the smooth positive functions, whereas in the insulating regime the fluctuations are determined by the squares of the separated delta-peaks and thus diverge as the width of the delta peaks goes to zero. Thus, we have the criterion

$$
\lim _{b(\omega) \rightarrow 0} \lim _{\mathcal{V} \rightarrow \infty} \frac{\left\langle[\delta \Gamma(\epsilon)]^{2}\right\rangle}{\langle\Gamma(\epsilon)\rangle^{2}}=\left\{\begin{array}{cc}
\text { finite; } & \text { metal } \\
\infty ; & \text { insulator }
\end{array}\right.
$$

Another way to address the same problem is to investigate the distribution function $P(\Gamma)$, see Fig. 6(c-d). One finds by simple inspection of Fig. 6(a-b)

$$
\lim _{b(\omega) \rightarrow 0} \lim _{\mathcal{V} \rightarrow \infty} P(\Gamma>0)=\left\{\begin{array}{cc}
>0 ; & \text { metal } \\
0 ; & \text { insulator. }
\end{array}\right.
$$


For us, it will be more technically convenient to perform the Laplace transform and calculate the characteristic function

$$
W(s)=\langle\exp [-s \Gamma(\epsilon)]\rangle
$$

where the precise definition of the averaging procedure is given in Sec. 6.1. The criterion (65) [see also Fig. 6(e-f)] of the insulating phase translates into

$$
\lim _{b \rightarrow 0} \lim _{\mathcal{V} \rightarrow \infty} W(s)=1
$$

for any fixed $s>0$.

Closing this subsection, we emphasize that the transition occurs as a function of temperature (which corresponds to the extensive energy of the many-body state) and not as a function of $\epsilon$ which charaterizes the energy of the oneparticle excitation on top of this many-body state. The latter energy is not an extensive quantity and can not be a characteristic of any phase transition, in contrast with conclusions of Ref. [16] for a finite-size system.

\subsection{Self-Consistent Born Approximations (SCBA and ImSCBA).}

In this subsection we introduce our main approximation for the summation of the infinite series of perturbative expansion. We will discuss motivation for this approximation here in quite loose terms and justify it further in Sec. 7.

Contributions to the self-energy shown in Figs. 4,5 bear different physical significance. For instance, the Hartree-Fock diagrams (b), (d2) of Fig. 4, and diagrams (3) of Fig. 5 characterize the self-consistent one-particle spectrum. As we explained before, the structure of this spectrum is not relevant for the transition. On the contrary, diagrams (c1) and (c3) of Fig. 4 can lead to irreversible processes, and the appearance of the imaginary part of those diagrams signals the metal-insulator transition. Thus, those diagrams will be taken into account.

The third order diagrams, Fig. 4d, describe the effect of the change of the selfconsistent potential on the tunneling process (d1); effect of the tunneling on the self-consitent potential (d3); and the effect of the self-consistent potential on the spectrum of the decay channel. These diagrams will be neglected for the reasons explained above.

Fourth order diagrams Fig. 5 describe further potentially irreversible process: tunneling out of the localization cell (1); three-particle production with the consequent tunneling (3); five-particle production (5). Those contributions must be taken into account. On the other hand, diagrams (2) and (4) once 
(a)

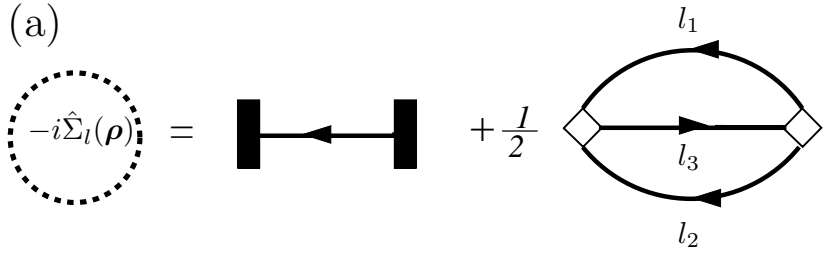

(b)

$\boldsymbol{1}+\underset{(l, \rho)}{1}+\mathbf{1}$

(1)

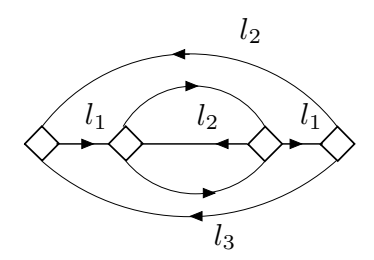

(2)

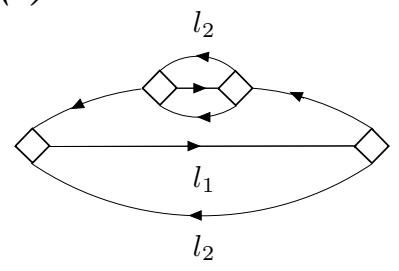

(c)

(1)

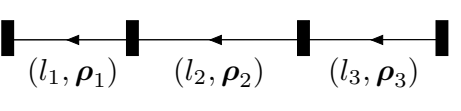

$\left(l_{1}, \boldsymbol{\rho}_{1}\right) \neq\left(l_{3}, \boldsymbol{\rho}_{3}\right) ;\left(l_{2}, \boldsymbol{\rho}_{2}\right) \neq(l, \boldsymbol{\rho})$

(2)

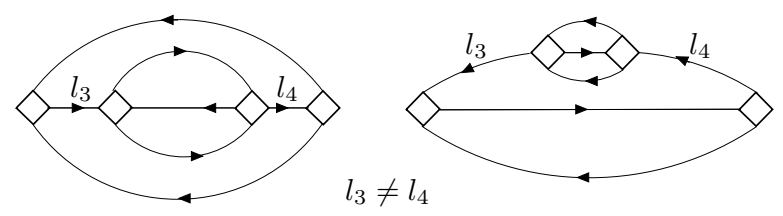

(3)

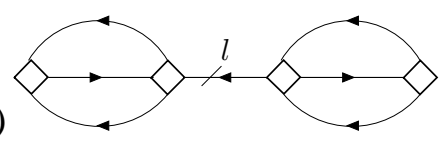

(4)

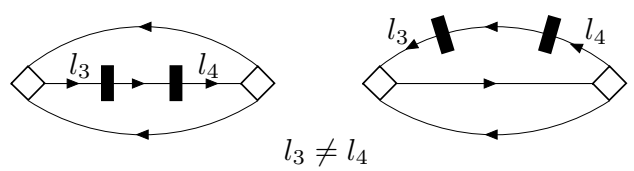

Fig. 7. a) Self-consistent Born approximation. The thick fermionic lines are defined in Fig. 4a. b) Spurious contributions of the fourth order generated by iteration of SCBA: b. 1 contradicts the definition of the self-energy; b.2 contains the intermediate particles in the same states; such contributions are cancelled by diagrams (5.1a) and (5.2a)-(5.2c) of Fig. 4. c) Fourth order tunneling and interaction contributions not taken into account by SCBA. These contributions have a random sign. 
again represent the self-consistent potential affecting the lower order processes (c1), (c3) of Fig 4. Diagrams (6.1), (6.2) of Fig. 5 describe the interaction of the quasiparticles created by the lower-order process (c3) of Fig. 4. Such effect has the same physical significance and the relative value as the effect of the self-consistent potential and will be neglected. The effect of those diagrams will be estimated in Sec. 7. Finally, diagrams (5.1a), and (5.2a)-(5.2c) are exchange counterparts of the main diagrams (5.1)-(5.2) [we will elaborate it further in Sec. 7]. Their role is to cancel out contributions in (5.1), (5.2) which are forbidden by Pauli principle (more than one particle in one intermediate state) and also to give the random sign interference corrections. The latter corrections will be neglected here and estimated further in Sec. 7 .

The above discussion of the lowest orders of the perturbation theory suggests the following prescription for the summation of the leading series: (i) we take into account only the even orders of the perturbation theory; (ii) we require that each contribution maximize the sum of the tunneling events and the extra quasiparticle production; (iii) we neglect contributions with random sign. By inspection, one can see that such series with the correct combinatorial coefficients is generated by the self-consistent Born approximation (SCBA) shown on Fig. 7a.

Iterations of the SCBA equations also produce spurious contributions shown on Fig. 7b. For the SCBA scheme to be valid, we will make sure that those spurious contributions are always smaller than those responsible for the final result.

We calculate the self-energy (Fig. 7a) according to the rules of Fig. 3. We neglect the shifts $\delta \xi_{l}(\boldsymbol{\rho})$ in accord with our previous discussion, i.e., we make the additional approximation to the usual SCBA scheme

$$
\Sigma_{l}^{(S C B A)}(\boldsymbol{\rho} ; \epsilon) \rightarrow i \operatorname{Im} \Sigma_{l}^{(S C B A)}(\boldsymbol{\rho} ; \epsilon)
$$

We will refer to approximation (68) as Im-Self-Consistent-Born-Approximation (ImSCBA). In the two subsequent sections, we will work only within this approximation.

Using spectral representation (61) and (62) and performing the integration 
over the intermediate energies, we obtain

$$
\begin{aligned}
& \Gamma_{l}(\epsilon)= \Gamma_{l}^{(e l)}(\epsilon)+\Gamma_{l}^{(i n)}(\epsilon)+\Gamma_{l}^{(\text {bath })}(\epsilon) ; \\
& \Gamma_{l}^{(e l)}(\epsilon, \boldsymbol{\rho})= \pi I^{2} \delta_{\zeta}^{2} \sum_{l_{1}, \boldsymbol{a}} A_{l_{1}}(\epsilon, \boldsymbol{\rho}+\boldsymbol{a}) ; \\
& \Gamma_{l}^{(i n)}(\epsilon)= \pi \lambda^{2} \delta_{\zeta}^{2} \sum_{l_{1}, l_{2}, l_{3}} Y_{l_{1}, l_{2}}^{l_{3}, l} \int \mathrm{d} \epsilon_{1} \mathrm{~d} \epsilon_{2} \mathrm{~d} \epsilon_{3} A_{l_{1}}\left(\epsilon_{1}\right) A_{l_{2}}\left(\epsilon_{2}\right) A_{l_{3}}\left(\epsilon_{3}\right) \\
& \times \delta\left(\epsilon-\epsilon_{1}-\epsilon_{2}+\epsilon_{3}\right) F_{l_{1}, l_{2} ; l_{3}}^{\Rightarrow}\left(\epsilon_{1}, \epsilon_{2} ; \epsilon_{3}\right) ; \\
& \Gamma_{l}^{(b a t h)}(\epsilon)= \frac{1}{2} \int \mathrm{d} \omega \omega b(\omega) A_{l}(\epsilon-\omega)\left[\operatorname{coth} \frac{\omega}{2 T}+n_{l}(\epsilon-\omega)\right] \\
& A_{l}(\epsilon)= \frac{\pi^{-1} \Gamma_{l}(\epsilon)}{\left[\epsilon-\xi_{l}\right]^{2}+\left[\Gamma_{l}(\epsilon)\right]^{2}} \\
& Y_{l_{1}, l_{2}}^{l_{3}, l} \equiv \frac{1}{2}\left[\Upsilon\left(\frac{\xi_{l_{2}}-\xi_{l}}{\delta_{\zeta}}\right) \Upsilon\left(\frac{\xi_{l_{1}}-\xi_{l_{3}}}{\delta_{\zeta}}\right)-\Upsilon\left(\frac{\xi_{l_{1}}-\xi_{l}}{\delta_{\zeta}}\right) \Upsilon\left(\frac{\xi_{l_{2}}-\xi_{l_{3}}}{\delta_{\zeta}}\right)\right]^{2} \\
& F_{l_{1}, l_{2} ; l_{3}}^{\Rightarrow}\left(\epsilon_{1}, \epsilon_{2} ; \epsilon_{3}\right)=\frac{1}{4}\left\{1+n_{l_{1}}\left(\epsilon_{1}\right) n_{l_{2}}\left(\epsilon_{2}\right)-n_{l_{3}}\left(\epsilon_{3}\right)\left[n_{l_{1}}\left(\epsilon_{1}\right)+n_{l_{2}}\left(\epsilon_{2}\right)\right]\right\} ;
\end{aligned}
$$

where $\eta$ is defined in Eq. (63), and we utilized the notation used in Eqs. (48) for the nearest neighbours. Everywhere, the coordinate $\boldsymbol{\rho}$ and time $t$ are assumed to be same in all terms in the equations unless it is specified explicitly otherwise.

Equations (69a) form a closed set for finding the decay rate for fixed occupation numbers $n_{l}(\epsilon, \boldsymbol{\rho})$. In the delocalized regime the time evolution of those occupation numbers is governed by the ImSCBA version of the kinetic eqution (57). Calculating the Keldysh component of Fig. 7a and using Eqs. (58) and (63), we find

$$
\begin{aligned}
\partial_{t} n_{l}(\epsilon)= & \mathrm{St}_{l}^{(e l)}+\mathrm{St}_{l}^{(i n)}+\mathrm{St}_{l}^{(\text {bath })} \\
\mathrm{St}_{l}^{(e l)}= & 2 \pi I^{2} \delta_{\zeta}^{2} \sum_{l_{1}, \boldsymbol{a}} A_{l_{1}}(\epsilon, \boldsymbol{\rho}+\boldsymbol{a})\left[n_{l_{1}}(\epsilon, \boldsymbol{\rho}+\boldsymbol{a})-n_{l}(\epsilon, \boldsymbol{\rho})\right] ; \\
\mathrm{St}_{l}^{(i n)}= & 2 \pi \lambda^{2} \delta_{\zeta}^{2} \sum_{l_{1}, l_{2}, l_{3}} Y_{l_{1}, l_{2}}^{l_{3}, l} \int \mathrm{d} \epsilon_{1} \mathrm{~d} \epsilon_{2} \mathrm{~d} \epsilon_{3} A_{l_{1}}\left(\epsilon_{1}\right) A_{l_{2}}\left(\epsilon_{2}\right) A_{l_{3}}\left(\epsilon_{3}\right) \delta\left(\epsilon-\epsilon_{1}-\epsilon_{2}+\epsilon_{3}\right) \\
& \times\left[-n_{l}(\epsilon) F_{l_{1}, l_{2} ; l_{3}}^{\Rightarrow}\left(\epsilon_{1}, \epsilon_{2} ; \epsilon_{3}\right)+F_{l_{1}, l_{2} ; l_{3}}^{\Leftarrow}\left(\epsilon_{1}, \epsilon_{2} ; \epsilon_{3}\right)\right] ; \\
F_{l_{1}, l_{2} ; l_{3}}^{\Leftarrow} & \left(\epsilon_{1}, \epsilon_{2} ; \epsilon_{3}\right)=\frac{1}{4}\left\{-n_{l_{3}}\left(\epsilon_{3}\right)\left[1+n_{l_{1}}\left(\epsilon_{1}\right) n_{l_{2}}\left(\epsilon_{2}\right)\right]+\left[n_{l_{1}}\left(\epsilon_{1}\right)+n_{l_{2}}\left(\epsilon_{2}\right)\right]\right\} ; \\
\mathrm{St}_{l}^{(b a t h)}= & \int \mathrm{d} \omega \omega b(\omega) A_{l}(\epsilon-\omega) \\
& \times\left\{\operatorname{coth} \frac{\omega}{2 T_{b}}\left[n_{l}(\epsilon-\omega)-n_{l}(\epsilon)\right]+1-n_{l}(\epsilon-\omega) n_{l}(\epsilon)\right\}
\end{aligned}
$$

Equation (69b) is the usual quantum Boltzmann equation written in terms of the exact (not averaged) single electron levels $\xi_{l}(\boldsymbol{\rho})$. As any Boltzmann equa- 
tion, it must respect the fundamental symmetries of the system: conservation of the number of particles for any collision; conservation of electron energy for the processes involving the electrons only; and the conservation of the number of particles for a given energy for elastic collisions. It is straightforward to check that the collision integral indeed possesses the desired properties:

$$
\begin{aligned}
& \sum_{l, \rho} A_{l}(\epsilon, \boldsymbol{\rho}) \mathrm{St}_{l}^{(e l)}(\epsilon, \boldsymbol{\rho})=0 ; \\
& \sum_{l} \int \mathrm{d} \epsilon A_{l}(\epsilon, \boldsymbol{\rho}) \mathrm{St}_{l}^{(i n)}(\epsilon, \boldsymbol{\rho})=0 ; \\
& \sum_{l} \int \mathrm{d} \epsilon \epsilon A_{l}(\epsilon, \boldsymbol{\rho}) \mathrm{St}_{l}^{(i n)}(\epsilon, \boldsymbol{\rho})=0 ; \\
& \sum_{l} \int \mathrm{d} \epsilon A_{l}(\epsilon, \boldsymbol{\rho}) \mathrm{St}_{l}^{(b a t h)}(\epsilon, \boldsymbol{\rho})=0 .
\end{aligned}
$$

The properties (70) of the collision integrals enable one to write the continuity equations for the particle and energy densities $\mathcal{N}$ and $\mathcal{E}$, and introduce the corresponding currents $\boldsymbol{J}, \boldsymbol{J}_{\mathcal{E}}$ :

$$
\begin{aligned}
& \partial_{t} \mathcal{N}(\boldsymbol{\rho})+\operatorname{div} \boldsymbol{J}(\boldsymbol{\rho})=0 ; \\
& \partial_{t} \mathcal{E}(\boldsymbol{\rho})+\operatorname{div} \boldsymbol{J}_{\mathcal{E}}(\boldsymbol{\rho})=-Q_{b a t h}(\boldsymbol{\rho}) ;
\end{aligned}
$$

where $Q_{\text {bath }}$ is the thermal flow to the thermal bath; it will not be important for the further cosideration. The lattice version of the divergence of the currents is defined as

$$
\operatorname{div} \boldsymbol{J}(\boldsymbol{\rho})=\frac{1}{\zeta_{l o c}} \sum_{k=1}^{d}\left[J^{k}\left(\boldsymbol{\rho}+\frac{\boldsymbol{a}^{(k)}}{2}\right)-J^{k}\left(\boldsymbol{\rho}-\frac{\boldsymbol{a}^{(k)}}{2}\right)\right],
$$

and it becomes the usual divergence in the continuum limit. Index $k$ labels the direction in the cartesian coordianat system in $d$ dimensions. Vector $\boldsymbol{a}^{(k)}$ is the lattice vector along the $k$ th direction.

The densities are defined on the sites $\boldsymbol{\rho}$

$$
\left[\begin{array}{l}
\mathcal{N} \\
\mathcal{E}
\end{array}\right](\boldsymbol{\rho})=\frac{1}{\zeta_{l o c}^{d}} \int \mathrm{d} \epsilon\left[\begin{array}{l}
1 \\
\epsilon
\end{array}\right] \sum_{l} A_{l}(\epsilon, \boldsymbol{\rho}) \frac{1-n_{l}(\epsilon, \boldsymbol{\rho})}{2}
$$

whereas the currents are defined on the links $\boldsymbol{\rho}^{(k)}=\boldsymbol{\rho}+\boldsymbol{a}^{(k)} / 2$ :

$$
\begin{aligned}
{\left[\begin{array}{c}
J^{k} \\
J_{\mathcal{E}}^{k}
\end{array}\right]\left(\boldsymbol{\rho}^{(k)}\right)=} & \int \mathrm{d} \epsilon\left[\begin{array}{l}
1 \\
\epsilon
\end{array}\right] \sum_{l, l_{1}} A_{l}(\epsilon, \boldsymbol{\rho}) A_{l_{1}}\left(\epsilon, \boldsymbol{\rho}+\boldsymbol{a}^{(k)}\right) \\
& \times \frac{\pi I^{2} \delta_{\zeta}^{2}}{\zeta_{l o c}^{d-1}}\left[n_{l_{1}}\left(\epsilon, \boldsymbol{\rho}+\boldsymbol{a}^{(k)}\right)-n_{l}(\epsilon, \boldsymbol{\rho})\right],
\end{aligned}
$$


Finally, $\mathrm{St}_{l}^{(e l)}$ is nulled by any function $n_{l}(\epsilon ; \boldsymbol{\rho})=n(\epsilon)$, electron-electron inelastic collision integral $\mathrm{St}_{l}^{(i n)}$ is nulled by any Fermi finction $n_{l}(\epsilon ; \boldsymbol{\rho})=\tanh \frac{\epsilon-\mu(\boldsymbol{\rho})}{2 T(\boldsymbol{\rho})}$; and the $\mathrm{St}_{l}^{\left({ }^{(b a t h)}\right.}$ vanishes for $n_{l}(\epsilon ; \boldsymbol{\rho})=\tanh \frac{\epsilon-\mu(\boldsymbol{\rho})}{2 T_{b}}$.

The closed system of Eqs. (69) is a drastic (though parametrically justifiable) simplification in comparison with the original problem. However, it is still a non-linear system which depends on an infinite number of random energies $\xi_{l}(\boldsymbol{\rho})$. Substantial progress can be achieved within the statistical analysis. This analysis is a subject of two following sections.

\section{$5 \quad$ Stability and properties of the metallic phase.}

\subsection{Condition for stability}

The hallmark of the developed metallic phase is self-averaging of the kinetic coefficients, see Sec. 4.2. To establish a sufficient condition for the existence and stability of this phase, we assume that the inelastic decay rate is indeed self-averaging and then justify this assumption by explicit calculation of its mesoscopic fluctuations, see Fig. 8. As a result, we will see that this condition reduces to a certain integral inequality for the distribution function, see Eq. (77). We will also see that even when this condition is satisfied, the elastic rate still may not be self-averaging. This, however, will not violate the criterion (64), as the fluctuations of the elastic rate remain finite.

First, we take into account only the inelastic rate in the $A_{l}$ of Eq. (69a). We assume and justify a posteriori that the main contribution originates from

$$
\left|l-l_{i}\right|,\left|l_{i}-l_{j}\right| \gg 1, i, j=1,2,3 .
$$

Statistical averaging over the distribution of the levels $\xi_{l}$ can be performed independently,

$$
\left\langle\sum_{l}(\ldots)\right\rangle=\delta_{\zeta}^{-1} \int \mathrm{d} \xi_{l}(\ldots) .
$$

For the needed products of the spectral densities, see Eq. (69a), we find

$$
\begin{aligned}
& \left\langle\sum_{l} A_{l}(\epsilon)\right\rangle=\frac{1}{\delta_{\zeta}} ; \\
& \left\langle\sum_{l} A_{l}\left(\epsilon_{1}\right) A_{l}\left(\epsilon_{2}\right)\right\rangle=\frac{1}{\pi \delta_{\zeta}} \frac{2 \Gamma^{(i n)}\left(\epsilon_{1}\right)}{\left(\epsilon_{1}-\epsilon_{2}\right)^{2}+\left[2 \Gamma^{(i n)}\left(\epsilon_{1}\right)\right]^{2}},
\end{aligned}
$$




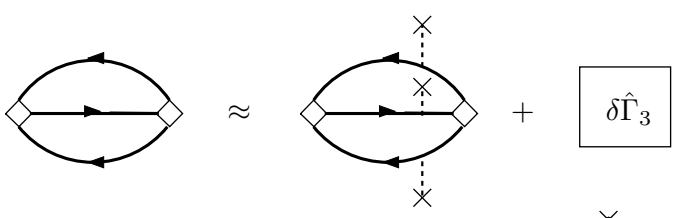

a)

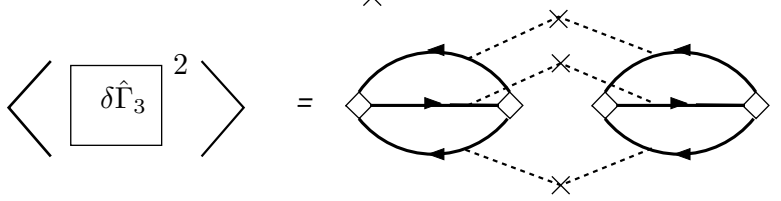

b)

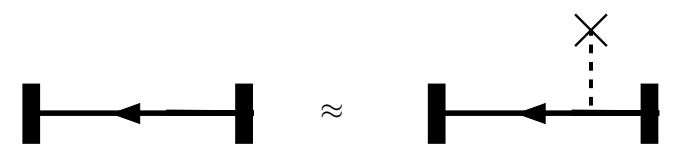

Fig. 8. a) Simplification of the self-consistent Born approximation at $T>T_{i n}$. b) The self-averaging of the hopping term at $T>T_{e l}$. Cross with the dashed line(s) stands for the averaging of the corresponding Green function (product of the Green functions) over the realization of random energies $\xi_{l}(\boldsymbol{\rho})$, see Eq. (48a).

where we assumed that $\Gamma(\epsilon)$ is a smooth function on the scale of $\Gamma(0)$.

Averaging Eq. (69a) with the help of Eq. (73), see also Fig. 8a, and assuming that the distribution functions $n_{l}(\epsilon, \boldsymbol{\rho})$ do not depend explicitly on the orbital index $l$, we find: ${ }^{11}$

$$
\begin{aligned}
\left\langle\Gamma_{l}^{(i n)}(\epsilon)\right\rangle= & \frac{\pi \lambda^{2}}{\delta_{\zeta}} \int \mathrm{d} \epsilon_{1} \mathrm{~d} \omega F^{\Rightarrow}\left(\epsilon_{1}+\omega, \epsilon_{1} ; \epsilon-\omega\right) \\
& \times\left[\Upsilon^{4}\left(\frac{\omega}{\delta_{\zeta}}\right)-\Upsilon^{2}\left(\frac{\omega}{\delta_{\zeta}}\right) \Upsilon^{2}\left(\frac{\epsilon_{1}+\omega-\epsilon}{\delta_{\zeta}}\right)\right] .
\end{aligned}
$$

As we will see shortly, the metallic regime is realized when the characteristic energy scale of the distribution function is much larger than $M \delta_{\zeta}$ [Eq. (48h)] typical transferred energy in Eq. (74). Under such conditions, the second term in the second line of this equation is more restrictive on the phase volume and that is why it can be neglected:

$$
\left\langle\Gamma_{l}^{(i n)}(\epsilon)\right\rangle=\frac{\pi \lambda^{2}}{\delta_{\zeta}} \int \mathrm{d} \epsilon_{1} \mathrm{~d} \omega \Upsilon^{4}\left(\frac{\omega}{\delta_{\zeta}}\right) F^{\Rightarrow}\left(\epsilon_{1}+\omega, \epsilon_{1} ; \epsilon-\omega\right) .
$$

Calculating mesoscopic fluctuations of the inelastic rate shown in Fig. 8b with the help of Eq. (73), and keeping the terms with the largest phase volume we

${ }^{11}$ All the formulas of Sec. 5 are written under the condition $\left\langle\Gamma_{l}^{(i n)}(\epsilon)\right\rangle \ll M \delta_{\zeta}$. In this case one can neglect the dependence on the index $l$ of $\Gamma_{l}(\epsilon), n_{l}(\epsilon)$, and the collision integral. Such dependence is present only for $\left|\epsilon-\xi_{l}\right| \gtrsim M \delta_{\zeta}$, while all physical properties are determined by the region $\left|\epsilon-\xi_{l}\right| \sim \Gamma^{(i n)}$; in particular, the distribution function enters the observables only as $\sum_{l} A_{l}(\epsilon) n_{l}(\epsilon)$. 
find

$$
\left\langle\left[\delta \Gamma_{l}^{(i n)}(\epsilon)\right]^{2}\right\rangle=\frac{\pi \lambda^{4} \delta_{\zeta}}{2} \int \mathrm{d} \epsilon_{1} \mathrm{~d} \omega \frac{\Upsilon^{8}\left(\omega / \delta_{\zeta}\right)\left[F^{\Rightarrow}\left(\epsilon_{1}+\omega, \epsilon_{1} ; \epsilon-\omega\right)\right]^{2}}{\Gamma^{(i n)}(\epsilon+\omega)+\Gamma^{(i n)}\left(\epsilon_{1}\right)+\Gamma^{(i n)}\left(\epsilon_{1}-\omega\right)} .
$$

Our initial assumption that the inelastic rate is self-avergaing is justified provided that

$$
\left\langle\left[\delta \Gamma_{l}^{(i n)}(\epsilon)\right]^{2}\right\rangle \lesssim\left[\left\langle\Gamma_{l}^{(i n)}(\epsilon)\right\rangle\right]^{2}
$$

According to Eqs. (75) and Eq. (76), both sides of this inequality are determined by the distribution function only, so that Eq. (77) is a sufficient (but not necessary) condition for the arbitrary non-equilibrium state to be metallic.

For the thermal distribution $n(\epsilon)=\tanh \frac{\epsilon}{2 T}$, the explicit expressions can be obtained. One finds

$$
\left\langle\Gamma^{(i n)}\right\rangle=\pi \lambda^{2} M T
$$

where $M$ is the coefficient defined in Eq. (48h), and

$$
\left\langle\left(\delta \Gamma^{(i n)}\right)^{2}\right\rangle=\frac{\pi \lambda^{4} M \delta_{\zeta}^{2} T}{36\left\langle\Gamma^{(i n)}\right\rangle}
$$

The condition (77), thus, reduces to the lower bound for the temperature

$$
T \gtrsim T_{i n} \equiv \frac{\delta_{\zeta}}{6 \pi \lambda M}
$$

To complete our discussion of the properties of inelastic rate, we justify our assumptions. To check the condition (72). we anlalyze the structure of the energy integrals in Eqs. (74)-(76) and find $\left|l-l_{1}\right|,\left|l_{2}-l_{3}\right| \simeq M \gg 1,\left|l-l_{3}\right| \simeq$ $T / \delta_{\zeta} \gg M$, which is consistent with Eq. (72). Deriving Eq. (78) we assumed $T \gg M \delta_{\zeta}$. It is consitent with Eq. (80) provided that the condition (48h) is fullfilled.

Let us turn now to the properties of the elastic decay rate, $\Gamma^{(e l)}$, of Eq. (69a)

$$
\begin{aligned}
& \Gamma_{l}^{(e l)}(\epsilon, \boldsymbol{\rho})=\pi \delta_{\zeta}^{2} I^{2} \sum_{\boldsymbol{a}} A(\epsilon, \boldsymbol{\rho}+\boldsymbol{a}) \\
& A(\epsilon, \boldsymbol{\rho})=\frac{1}{\pi} \sum_{l_{1}} \frac{\Gamma^{(i n)}(\epsilon)}{\left[\epsilon-\xi_{l_{1}}(\boldsymbol{\rho})\right]^{2}+\left[\Gamma^{(i n)}(\epsilon)\right]^{2}} .
\end{aligned}
$$

Let us note that only the inelastic width enters the right-hand sides of these equations. Further iterations of the elastic processes in expression for $A_{l}(\boldsymbol{\rho}+\boldsymbol{a})$, see Eq. (69a), generate either terms small as $I^{2}\left(\Gamma^{(i n)}(\epsilon) / \delta_{\zeta}\right) \ll 1$ (originating from off-resonant levels), or the divergent term corresponding to the elastic 

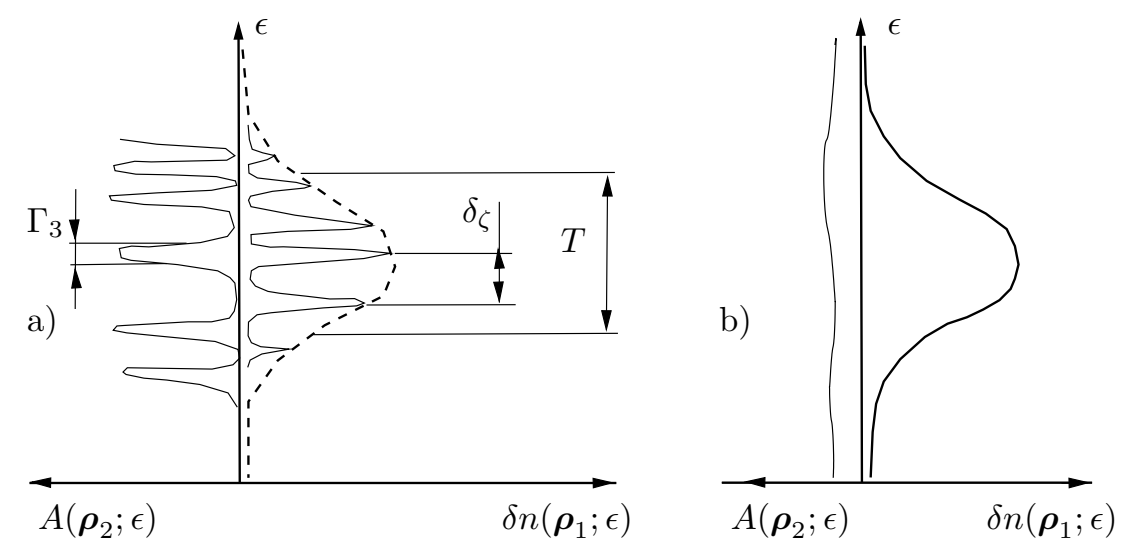

Fig. 9. Sketches of the shapes of the non-equilibrium influx function $\delta n\left(\boldsymbol{\rho}_{1}, \epsilon\right)$ and the spectral density of the other neighbours $A\left(\boldsymbol{\rho}_{2} ; \epsilon\right) \equiv \sum_{l} A_{l}\left(\boldsymbol{\rho}_{2} ; \epsilon\right)$ of the other neighbour; for a) low-temperature metal $T_{i n}<T<T_{e l}$; b) $T>T_{e l}$. See text for the further explanation.

return on the same level. The latter contribution, however, corresponds to the spurious diagram Fig. 7b.1 and must be discarded.

Average and fluctuations of the elastic rate (81) is calculated with the help of the Eq. (73) and we find

$$
\left\langle\Gamma^{(e l)}\right\rangle=(2 d) \pi I^{2} \delta_{\zeta} ; \quad\left\langle\left[\Gamma^{(e l)}(\epsilon)\right]^{2}\right\rangle=\frac{d \pi I^{4} \delta_{\zeta}^{3}}{\Gamma^{(i n)}(\epsilon)}
$$

where $2 d$ is the number of the nearest neighbors.

Using Eq. (78), we find

$$
\frac{\left\langle\left[\Gamma^{(e l)}\right]^{2}\right\rangle}{\left\langle\Gamma^{(e l)}\right\rangle^{2}}=\frac{4 T_{e l}}{T}, \quad T_{e l}=\frac{\delta_{\zeta}}{16 \pi^{2} d M \lambda^{2}} \simeq \frac{T_{i n}}{\lambda},
$$

where the numerical factor is chosen for the convenience in the further formulas, At $T \gg T_{e l}$ the level discreteness plays no role, so that both elastic and inelastic decay rates are self-averaging, see also Fig. 9. At $T_{i n} \ll T \ll T_{\text {el }}$ only $\Gamma^{(i n)}$ is self-averaging, while the fluctuations of $\Gamma^{(e l)}$ are large compared to the average. However, they are finite, so that the system is in the metallic state according to criterion (64). 


\subsection{Kinetic equation and transport coefficients.}

Having studied the statistical distribution of the decay rates, we are ready to apply the same ideas to the kinetic equation:

$$
\begin{array}{rl}
\partial_{t} & n(\epsilon, \boldsymbol{\rho})=2 \pi I^{2} \delta_{\zeta}^{2} \sum_{\boldsymbol{a}} A(\epsilon, \boldsymbol{\rho}+\boldsymbol{a})[n(\epsilon, \boldsymbol{\rho}+\boldsymbol{a})-n(\epsilon, \boldsymbol{\rho})] \\
+ & \frac{\pi \lambda^{2}}{2} \int \mathrm{d} \omega \mathrm{d} \epsilon_{1} \Upsilon^{4}\left(\frac{\omega}{\delta_{\zeta}}\right) A(\epsilon+\omega, \boldsymbol{\rho}) \\
\quad \times\left\{[n(\epsilon+\omega, \boldsymbol{\rho})-n(\epsilon, \boldsymbol{\rho})]\left[1-n\left(\epsilon_{1}, \boldsymbol{\rho}\right) n\left(\epsilon_{1}-\omega, \boldsymbol{\rho}\right)\right]\right. \\
\left.\quad+[1-n(\epsilon, \boldsymbol{\rho}) n(\epsilon+\omega, \boldsymbol{\rho})]\left[n\left(\epsilon_{1}-\omega, \boldsymbol{\rho}\right)-n\left(\epsilon_{1}, \boldsymbol{\rho}\right)\right]\right\} .
\end{array}
$$

Equation (84) enables us to find the kinetic coefficients in the system. We look for the distribution function in the form

$$
n(\epsilon, \boldsymbol{\rho}, t)=\tanh \frac{\epsilon}{2 T}+\Phi(\epsilon, \boldsymbol{\rho}, t)+\varphi(\epsilon, \boldsymbol{\rho}, t),
$$

where the function $\Phi(\epsilon, \boldsymbol{\rho}, t)$ describes the shape of the distribution function on the energy scale $\epsilon \gtrsim M \delta_{\zeta}$, whereas the function $\varphi(\epsilon, \boldsymbol{\rho})$ encodes the structure on the scale $\epsilon \sim \Gamma^{(i n)}, \delta_{\zeta}$. Namely, we impose the condition

$$
\langle\varphi(\epsilon, \boldsymbol{\rho})\rangle_{\epsilon} \equiv \int_{\epsilon-\Delta}^{\epsilon+\Delta} \frac{\mathrm{d} \epsilon_{1}}{2 \Delta} \varphi\left(\epsilon_{1}, \boldsymbol{\rho}\right)=0
$$

where $\Delta$ is an energy interval $\delta_{\zeta} \ll \Delta \lesssim M \delta_{\zeta}$. We substitute Eq. (85) into Eqs. (84) and linearize with respect to $\Phi$ and $\varphi$. For the smooth part of the distribution function we find

$$
\begin{aligned}
\partial_{t} & \Phi(\epsilon, \boldsymbol{\rho})=2 \pi I^{2} \delta_{\zeta} \sum_{\boldsymbol{a}}[\Phi(\epsilon, \boldsymbol{\rho}+\boldsymbol{a})-\Phi(\epsilon, \boldsymbol{\rho})] \\
& +2 \pi I^{2} \delta_{\zeta}^{2} \sum_{\boldsymbol{a}}\langle A(\epsilon, \boldsymbol{\rho}+\boldsymbol{a})[\varphi(\epsilon, \boldsymbol{\rho}+\boldsymbol{a})-\varphi(\epsilon, \boldsymbol{\rho})]\rangle_{\epsilon} \\
& +\widehat{\mathrm{St}}_{\Phi} \Phi(\epsilon, \boldsymbol{\rho})+\left\langle\widehat{\mathrm{St}}_{\varphi} \varphi(\epsilon, \boldsymbol{\rho})\right\rangle_{\epsilon} .
\end{aligned}
$$

Deriving Eq. (87a) we used the fact that $\langle A(\epsilon)\rangle_{\epsilon}=1 / \delta_{\zeta}$ is a self-averaging quantity.

The smooth part, $\Phi$, contains, in particular, density and energy density which propagate diffusively through the system. In contrast, the oscillatory contribution decays due to the inelastic processes. Thus, function $\varphi$ can be considered 
in the stationary limit

$$
\begin{aligned}
0= & 2 \pi I^{2} \delta_{\zeta}^{2} \sum_{\boldsymbol{a}}\|A(\epsilon, \boldsymbol{\rho}+\boldsymbol{a})\|_{o s c}[\Phi(\epsilon, \boldsymbol{\rho}+\boldsymbol{a})-\Phi(\epsilon, \boldsymbol{\rho})] \\
& +2 \pi I^{2} \delta_{\zeta}^{2} \sum_{\boldsymbol{a}}\|A(\epsilon, \boldsymbol{\rho}+\boldsymbol{a})[\varphi(\epsilon, \boldsymbol{\rho}+\boldsymbol{a})-\varphi(\epsilon, \boldsymbol{\rho})]\|_{o s c} \\
& +\left\|\widehat{\operatorname{St}}_{\varphi} \varphi(\epsilon, \boldsymbol{\rho})\right\|_{o s c}
\end{aligned}
$$

where we introduced the notation for the oscillatory part of the expression

$$
\|\ldots\|_{o s c} \equiv \cdots-\langle\ldots\rangle_{\epsilon}
$$

The smooth part of the linearized collision integral $\widehat{\mathrm{St}}_{\varphi} \varphi$ is given by

$$
\begin{aligned}
\left\langle\widehat{\operatorname{St}}_{\varphi} \varphi(\epsilon)\right\rangle_{\epsilon} & =2 \pi \lambda^{2} T \int \mathrm{d} \omega \Upsilon^{4}\left(\frac{\omega}{\delta_{\zeta}}\right)\langle A(\epsilon+\omega)[\varphi(\epsilon+\omega)-\varphi(\epsilon)]\rangle_{\epsilon} \\
& \approx 2 \pi \lambda^{2} T \int \mathrm{d} \omega \Upsilon^{4}\left(\frac{\omega}{\delta_{\zeta}}\right)\langle A(\epsilon+\omega) \varphi(\epsilon+\omega)\rangle_{\epsilon} \\
& \approx 2 \Gamma^{(i n)} \delta_{\zeta}\langle A(\epsilon) \varphi(\epsilon)\rangle_{\epsilon} .
\end{aligned}
$$

Here we used the fact that $\langle A(\epsilon+\omega) \varphi(\epsilon)\rangle_{\epsilon}=\langle A(\epsilon+\omega)\rangle_{\epsilon}\langle\varphi(\epsilon)\rangle_{\epsilon}=0$, for $|\omega| \gtrsim \delta_{\zeta}$ and the contribution to the integral is determined by $|\omega| \simeq M \delta_{\zeta}$. We also used the expression for the inelastic rate (78). By the same token, we find the oscillatory part as

$$
\left\|\hat{\mathrm{St}}_{\varphi} \varphi(\epsilon)\right\|_{o s c}=\frac{2 \pi \lambda^{2} T}{\delta_{\zeta}} \int \mathrm{d} \omega \Upsilon^{4}\left(\frac{\omega}{\delta_{\zeta}}\right)[\varphi(\epsilon+\omega)-\varphi(\epsilon)] \approx-2 \Gamma^{(i n)} \varphi(\epsilon) .
$$

We remind the reader that the relationship $T \gg T_{\text {in }} \gg M \delta_{\zeta}$ is widely used, see also Eq. (80). Finally, the shape of the smooth distriution function is stabilized by the linearized collision integral

$$
\begin{aligned}
\widehat{\mathrm{St}}_{\Phi} \Phi(\epsilon)= & \frac{2 \pi \lambda^{2}}{\delta_{\zeta}} \int \mathrm{d} \omega \Upsilon^{4}\left(\frac{\omega}{\delta_{\zeta}}\right)\left\{\frac{\omega}{2} \operatorname{coth} \frac{\omega}{2 T}[\Phi(\epsilon+\omega)-\Phi(\epsilon)]\right. \\
& +\frac{\omega}{2}\left[\Phi(\epsilon+\omega) \tanh \frac{\epsilon}{2 T}+\Phi(\epsilon) \tanh \frac{\epsilon+\omega}{2 T}\right] \\
& +\frac{1}{4}\left(\tanh \frac{\epsilon-\omega}{2 T}-\tanh \frac{\epsilon}{2 T}\right) \\
& \left.\times \int \mathrm{d} \epsilon_{1}\left[\Phi\left(\epsilon_{1}+\omega\right) \tanh \frac{\epsilon_{1}}{2 T}+\Phi\left(\epsilon_{1}\right) \tanh \frac{\epsilon_{1}+\omega}{2 T}\right]\right\} .
\end{aligned}
$$

This linear operator has two zero modes

$$
\Phi_{\mu}(\epsilon)=-\partial_{\epsilon} \tanh \frac{\epsilon}{2 T} ; \quad \Phi_{T}(\epsilon)=\partial_{T} \tanh \frac{\epsilon}{2 T},
$$


which reflect the conservation of number of particles and energy by the inelastic processes.

Because of the condition $T \gg M \delta_{\zeta}$, Eq. (87e) can be rewritten in the energy diffusion approximation

$$
\begin{aligned}
\widehat{\mathrm{St}}_{\Phi} \Phi(\epsilon)= & D^{(\epsilon)}\left\{\partial_{\epsilon}^{2} \Phi(\epsilon)+\frac{\partial_{\epsilon}}{2}\left[\Phi(\epsilon) \tanh \frac{\epsilon}{2 T}\right]\right. \\
& \left.-\frac{\partial_{\epsilon}^{2}}{4} \tanh \frac{\epsilon}{2 T} \int \mathrm{d} \epsilon_{1} \Phi\left(\epsilon_{1}\right) \tanh \frac{\epsilon_{1}}{2 T}\right\} ; \\
D^{(\epsilon)}= & \frac{\pi M^{3} \delta_{\zeta}^{2} \lambda^{2} T}{12} .
\end{aligned}
$$

The simple form of the collision integral (87d) enables us to solve Eq. (87b). We adopt the following relation

$$
\Gamma^{(i n)} \gg I \delta_{\zeta}
$$

which is automatically fullfilled ${ }^{12}$ for $T \gg T^{(i n)}$, see Eq. (80), and $I \simeq \lambda$. Then $\Gamma^{(i n)} \gg I^{2} \delta_{\zeta}^{2} A(\epsilon)$ and Eq. (87b) yields

$$
\begin{aligned}
\varphi(\epsilon, \boldsymbol{\rho}) & =\frac{\pi I^{2} \delta_{\zeta}^{2}}{\Gamma^{(i n)}} \sum_{\boldsymbol{a}}\|A(\epsilon, \boldsymbol{\rho}+\boldsymbol{a})\|_{o s c} \\
& \times[\Phi(\epsilon, \boldsymbol{\rho}+\boldsymbol{a})-\Phi(\epsilon, \boldsymbol{\rho})]\left[1+\mathcal{O}\left(\frac{I^{2} \delta_{\zeta}^{2}}{\left[\Gamma^{(i n)}\right]^{2}}\right)\right] .
\end{aligned}
$$

$\overline{12}$ The regime $\Gamma^{(i n)} \ll I \delta_{\zeta}$, which may occur for somewhat artificial for our model choice $I \gg \lambda$, corresponds to coherent oscillations of the population between two resonant levels. In this regime one should modify Eqs. (89) and (90) as

$$
\begin{aligned}
\varphi(\epsilon, \boldsymbol{\rho}) & =\sum_{\boldsymbol{a}}[\Phi(\epsilon, \boldsymbol{\rho}+\boldsymbol{a})-\Phi(\epsilon, \boldsymbol{\rho})] \\
\times & \frac{2 \pi I^{2} \delta_{\zeta}^{2} A(\epsilon, \boldsymbol{\rho}+\boldsymbol{a})}{2 \Gamma^{(i n)}+2 \pi I^{2} \delta_{\zeta}^{2}[A(\epsilon, \boldsymbol{\rho}+\boldsymbol{a})+A(\epsilon, \boldsymbol{\rho})]} \|_{o s c}, \\
\partial_{t} \Phi(\epsilon, \boldsymbol{\rho})-\widehat{\mathrm{St}}_{\Phi} \Phi(\epsilon, \boldsymbol{\rho})=\sum_{\boldsymbol{a}}[\Phi(\epsilon, \boldsymbol{\rho}+\boldsymbol{a})-\Phi(\epsilon, \boldsymbol{\rho})] & \\
\times & \left\langle\frac{2 \pi I^{2} \delta_{\zeta}^{2} A(\epsilon, \boldsymbol{\rho}) 2 \Gamma^{(i n)} A(\epsilon, \boldsymbol{\rho}+\boldsymbol{a})}{2 \Gamma^{(i n)}+2 \pi I^{2} \delta_{\zeta}^{2}[A(\epsilon, \boldsymbol{\rho}+\boldsymbol{a})+A(\epsilon, \boldsymbol{\rho})]}\right\rangle_{\epsilon} .
\end{aligned}
$$

One should also use the exact spectral densities for the resonant pairs, as obtained from the solution of a $2 \times 2$ problem. The results for the low- and high-temperature regimes remain unchanged, but an intermediate region appears. This regime is analogous to that studied in Ref. [25] for the conductivity due to the scattering of localized electrons on phonons. 
Substituting Eq. (89) into Eqs. (87c) and (87a), and keeping once again only the terms leading in $\left(I \delta_{\zeta} / \Gamma^{(i n)}\right)^{2}$, we find the equation for the smooth part of the distribution function

$$
\partial_{t} \Phi(\boldsymbol{\rho})=\widehat{\operatorname{St}}_{\Phi} \Phi(\boldsymbol{\rho})+2 \pi I^{2} \delta_{\zeta}^{3} \sum_{\boldsymbol{a}}\langle A(\epsilon, \boldsymbol{\rho}) A(\epsilon, \boldsymbol{\rho}+\boldsymbol{a})\rangle_{\epsilon}[\Phi(\boldsymbol{\rho}+\boldsymbol{a})-\Phi(\boldsymbol{\rho})],
$$

where we omitted the energy argument of the distribution function.

Equation (90) deserves some discussion. The first term on the right-hand side determines dynamics of the population at different energies $\epsilon$ on the site $\boldsymbol{\rho}$. The second term describes transport between neighboring sites. The inspection of this term shows that for $\Gamma^{(i n)} \ll \delta_{\zeta}$ this term is contributed mostly by rare overlaps of the peaks in the spectral densities on the neighboring sites, see Fig. 9. We will call such rare events "pin-holes". The typical energy separation between the pin-holes is $\delta_{\zeta}^{2} / \Gamma^{(i n)}$. Thus, for a given energy this term may not be self-averaging, and the calculation of the observable transport coefficients requires further statistical analysis. We note that the situation when transport is dominated by rare configurations is quite common in systems with strongly fluctuating local transmission, see Ref. [26].

To perform this analysis, we assume that the energy relaxation rate within each site is larger than the rate of the tunnelling into the neighbors (this assumption is justified for our model in Appendix A). In this case the solution is restricted to the zero modes of the inelastic collision integral (87f)

$$
\Phi(\epsilon, \boldsymbol{\rho} ; t)=\delta \mu(\boldsymbol{\rho}, t) \Phi_{\mu}(\epsilon)+\delta T(\boldsymbol{\rho}, t) \Phi_{T}(\epsilon)
$$

Substituting Eq. (91) into Eq. (90), performing the corresponding energy integration to utilize the properties $\int \mathrm{d} \epsilon \widehat{\operatorname{St}}_{\Phi} \Phi(\epsilon, \boldsymbol{\rho})=0$ and $\int \mathrm{d} \epsilon \epsilon \widehat{\operatorname{St}}_{\Phi} \Phi(\epsilon, \boldsymbol{\rho})=$ 0 , and neglecting thermopower and Peltier coefficients (which have random signs), we find

$$
\begin{aligned}
& \frac{e^{2}}{\delta_{\zeta}} \frac{\partial \mu(\boldsymbol{\rho})}{\partial t}=\sum_{\boldsymbol{a}} G\left(\boldsymbol{\rho}+\frac{\boldsymbol{a}}{2}\right)[\mu(\boldsymbol{\rho}+\boldsymbol{a})-\mu(\boldsymbol{\rho})] \\
& c_{V} \frac{\partial T(\boldsymbol{\rho})}{\partial t}=\sum_{\boldsymbol{a}} \mathcal{K}\left(\boldsymbol{\rho}+\frac{\boldsymbol{a}}{2}\right)[T(\boldsymbol{\rho}+\boldsymbol{a})-T(\boldsymbol{\rho})]
\end{aligned}
$$

where $c_{V}=\pi^{2} \nu T / 3$ is the specific heat per localization cell. The electrical conductance $G$ and the thermal conductance $\mathcal{K}$ are defined for each link $\boldsymbol{\rho}+\boldsymbol{a} / 2$ connecting two sites $\boldsymbol{\rho}$ and $\boldsymbol{\rho}+\boldsymbol{a}$. They are given by

$$
G\left(\boldsymbol{\rho}+\frac{\boldsymbol{a}}{2}\right)=\frac{2 \pi e^{2} I^{2}}{\hbar} \mathcal{B}_{\sigma}\left(\boldsymbol{\rho}+\frac{\boldsymbol{a}}{2}\right) ; \quad \mathcal{K}\left(\boldsymbol{\rho}+\frac{\boldsymbol{a}}{2}\right)=\frac{2 \pi^{3} e^{2} I^{2} T}{3 \hbar} \mathcal{B}_{\kappa}\left(\boldsymbol{\rho}+\frac{\boldsymbol{a}}{2}\right)
$$


where $\mathcal{B}_{\sigma, \kappa}$ are dimensionless random quantities determined by the overlaps of the densities of states:

$$
\begin{aligned}
\mathcal{B}_{\sigma, \kappa}\left(\boldsymbol{\rho}+\frac{\boldsymbol{a}}{2}\right) & =\delta_{\zeta}^{2} \int \frac{\mathrm{d} \epsilon}{2 T} \beta_{\sigma, \kappa}\left(\frac{\epsilon}{2 T}\right) A(\epsilon, \boldsymbol{\rho}) A\left(\epsilon, \boldsymbol{\rho}^{\prime}\right) \\
& =\sum_{l, l^{\prime}} \frac{\delta_{\zeta}}{2 T} \beta_{\sigma, \kappa}\left(\frac{\xi_{l}(\boldsymbol{\rho})}{2 T}\right) \frac{2 \delta_{\zeta} \Gamma^{(i n)} / \pi}{\left[\xi_{l}(\boldsymbol{\rho})-\xi_{l^{\prime}}\left(\boldsymbol{\rho}^{\prime}\right)\right]^{2}+\left[2 \Gamma^{(i n)}\right]^{2}},
\end{aligned}
$$

where $\boldsymbol{\rho}^{\prime} \equiv \boldsymbol{\rho}+\boldsymbol{a}$. We used the explicit expression Eq. (81) for $A(\epsilon, \boldsymbol{\rho})$ and the condition $\Gamma^{(i n)} \ll T$. Dimensionless functions $\beta_{\sigma, \kappa}(x)$ such that $\int \beta_{\sigma, \kappa}(x) \mathrm{d} x=$ 1 , are given by

$$
\beta_{\sigma}(x)=\frac{1}{2 \cosh ^{2} x}, \quad \beta_{\kappa}(x)=\frac{6 x^{2}}{\pi^{2} \cosh ^{2} x}
$$

Equations (92) are nothing but the description of a network of random conductors (thermal conductors). It is easy to check that the average of these quantities over the realizations of $\xi_{l}$ gives the temperature-independent result $\left\langle\mathcal{B}_{\sigma, \kappa}\right\rangle=1$. However, this result is meaningless as the observable conductivities are determined by typical rather than rare events. In fact, observable conductivity $\sigma$ and observable thermal conductivity $\kappa$ are given by

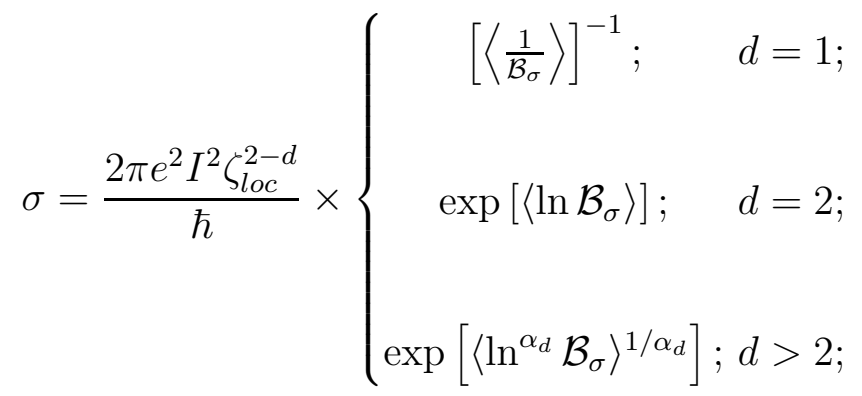

$$
\begin{aligned}
& \kappa=\frac{2 \pi^{3} e^{2} T I^{2} \zeta_{l o c}^{2-d}}{3 \hbar} \times\left\{\begin{array}{cc}
{\left[\left\langle\frac{1}{\mathcal{B}_{\kappa}}\right\rangle\right]^{-1} ;} & d=1 ; \\
\exp \left[\left\langle\ln \mathcal{B}_{\kappa}\right\rangle\right] ; & d=2 ; \\
\exp \left[\left\langle\ln ^{\alpha_{d}} \mathcal{B}_{\kappa}\right\rangle^{1 / \alpha_{d}}\right] ; d>2 ;
\end{array}\right.
\end{aligned}
$$

where $d$ is the dimensionality of the system and $\alpha_{d}$ are the numerical coefficients of the order of unity which are not known analytically for $d>2$. The formula for $d=1$ is the trivial result for the random resistors connected in series whereas the result for $d=2$ follows from the duality arguments [27].

The averages entering Eq. (93) can be immediately calculated if the charac- 
teristic functions $\tilde{P}_{\sigma, \kappa}(s)=\left\langle\mathrm{e}^{-s \mathcal{B}_{\sigma, \kappa}}\right\rangle$ of the distributions are known: ${ }^{13}$

$$
\left\langle\frac{1}{\mathcal{B}_{\sigma, \kappa}}\right\rangle=\int_{0}^{\infty} \mathrm{d} s \tilde{P}_{\sigma, \kappa}(s) ; \quad\left\langle\ln \mathcal{B}_{\sigma, \kappa}\right\rangle=\int_{0}^{\infty} \frac{\mathrm{d} s}{s}\left[\mathrm{e}^{-s}-\tilde{P}_{\sigma, \kappa}(s)\right]
$$

The characteristic functions $\tilde{P}_{\sigma, \kappa}(s)$ can be found from the definition (92c) by a straightforward calculation given in Appendix B:

$$
\tilde{P}_{\sigma, \kappa}(s)=\exp \left\{-\int_{-\infty}^{\infty} \mathrm{d} x\left[r S_{2}\left(\frac{s \beta_{\sigma, \kappa}(x)}{r}\right)\right]\right\}, \quad S_{2}(y)=y \mathrm{e}^{-y}\left[\mathcal{I}_{0}(y)+\mathcal{I}_{1}(y)\right]
$$

where $\mathcal{I}_{0}$ and $\mathcal{I}_{1}$ are the modified Bessel functions, and $\beta_{\sigma, \kappa}$ are given by Eq. (92d). The result is controlled by a dimensionless parameter

$$
r(T)=\frac{8 \pi \Gamma^{(i n)} T}{\delta_{\zeta}^{2}}=\frac{8 \pi^{2} \lambda^{2} M T^{2}}{\delta_{\zeta}^{2}}=\frac{1}{2 d}\left(\frac{T^{2}}{\delta_{\zeta} T_{e l}}\right) .
$$

The meaning of this parameter is the typical number of resonances in the energy strip $|\epsilon| \lesssim T$.

The result of numerical integration of Eqs. (94), (95) is plotted on Fig. 10. In two limiting cases $r \gg 1$ and $r \ll 1$ we have

$$
\begin{aligned}
& r \gg 1:\left\{\begin{aligned}
\tilde{P}_{\sigma}(s) \approx \mathrm{e}^{-s}\left[1+(1 / 6) s^{2} / r\right], \\
\tilde{P}_{\kappa}(s) \approx \mathrm{e}^{-s}\left[1+\left(7 / 10-6 / \pi^{2}\right) s^{2} / r\right],
\end{aligned}\right. \\
& r \ll 1: \quad\left\{\begin{array}{l}
\tilde{P}_{\sigma}(s) \approx \mathrm{e}^{-\sqrt{\pi r s}}, \\
\tilde{P}_{\kappa}(s) \approx \mathrm{e}^{-\sqrt{\left(192 \mathrm{G}^{2} / \pi^{3}\right) r s}},
\end{array}\right.
\end{aligned}
$$

where $\mathrm{G} \approx 0.916 \cdots$ is the Catalan's constant. Substituting Eq. (97) into Eq. (94) we find that for $r \gg 1, P_{\sigma, \kappa}(\mathcal{B})$ are strongly peaked around 1 , so that

$\overline{13}$ The average of the logarithm is calculated using the identity

$$
\int_{0}^{\infty} \mathrm{d} s \frac{\mathrm{e}^{-a s}-\mathrm{e}^{-b s}}{s}=\ln \frac{b}{a}
$$


the temperature indepedent Drude resistivity is restored:

$$
\begin{aligned}
& \sigma\left(T \gg \sqrt{\delta_{\zeta} T_{e l}}\right) \approx \sigma_{\infty}\left(1-\frac{2}{3} \frac{\delta_{\zeta} T_{e l}}{T^{2}}\right) \\
& \kappa\left(T \gg \sqrt{\delta_{\zeta} T_{e l}}\right) \approx \kappa_{\infty}(T)\left[1-\left(\frac{14}{5}-\frac{24}{\pi^{2}}\right) \frac{\delta_{\zeta} T_{e l}}{T^{2}}\right] \\
& \sigma_{\infty} \equiv \frac{2 \pi e^{2} I^{2} \zeta_{l o c}^{2-d}}{\hbar}, \quad \kappa_{\infty}(T) \equiv \frac{2 \pi^{3} e^{2} T I^{2} \zeta_{l o c}^{2-d}}{3 \hbar}
\end{aligned}
$$

In other words, the effect of localization of one-particle wave functions on transport is completely removed by the inelastic processes even in the "nonergodic" regime of $\Gamma^{(i n)} \ll \delta_{\zeta}$, where the peaks in the one-particle density of states are still well-resolved.

In the opposite case, $r \ll 1$ we have for $d=1,2$ :

$$
\sigma\left(T \ll \sqrt{\delta_{\zeta} T_{e l}}\right)=\sigma_{\infty} \frac{\pi}{4}\left(\frac{T^{2}}{\delta_{\zeta} T_{e l}}\right), \quad \kappa\left(T \ll \sqrt{\delta_{\zeta} T_{e l}}\right)=\kappa_{\infty}(T) \frac{48 \mathrm{G}^{2}}{\pi^{3}}\left(\frac{T^{2}}{\delta_{\zeta} T_{e l}}\right),
$$

(for larger dimensionalities the temperature dependence is the same but the numerical coefficients could not be found analytically).

These results have the following meaning. At $T \gg \sqrt{\delta_{\zeta} T_{e l}}$ the quantities are self-averaging and the result is temperature independent. At $T \ll \sqrt{\delta_{\zeta} T_{e l}}$ the electron on a site cannot explore enough pin-holes to find the rare resonant one which would determine the average. As a result, it chooses the best available pin-hole (i.e., the one with the smallest separation between the levels). The denominator in the Eq. (92c) can be estimated as $\left|\xi(\boldsymbol{\rho})-\xi\left(\boldsymbol{\rho}^{\prime}\right)\right| \simeq \delta_{\zeta} / n$, where $n \simeq T / \delta_{\zeta}$ is the number of the levels available for the electrons. As a result, the largest term entering into the sums in Eq. $(92 \mathrm{c})$ is $\simeq \Gamma^{(i n)} T \propto T^{2}$. It explains the power law dependence at low temperature.

It is instructive to investigate the validity of the Wiedemann-Frantz law. From Eq. (99) we find for the Lorentz number

$$
\frac{\mathrm{L}(T)}{\mathrm{L}_{0}} \equiv \frac{3 e^{2} \kappa(T)}{\pi^{2} \sigma(T) T}=\left\{\begin{array}{c}
1+\left(\frac{24}{\pi^{2}}-\frac{32}{15}\right) \frac{\delta_{\zeta} T_{e l}}{T^{2}}, T \gg \sqrt{\delta_{\zeta} T_{e l}} \\
\frac{192 \mathrm{G}^{2}}{\pi^{4}} \approx 1.65 \ldots, \quad T \ll \sqrt{\delta_{\zeta} T_{e l}}
\end{array}\right.
$$

The origin of the violation of the Wiedemann-Franz is the following. For the conductivity all the tunnelling pairs with the energies $\simeq T$ are roughly of the same importance. For the thermal conduction, however, the contributions 

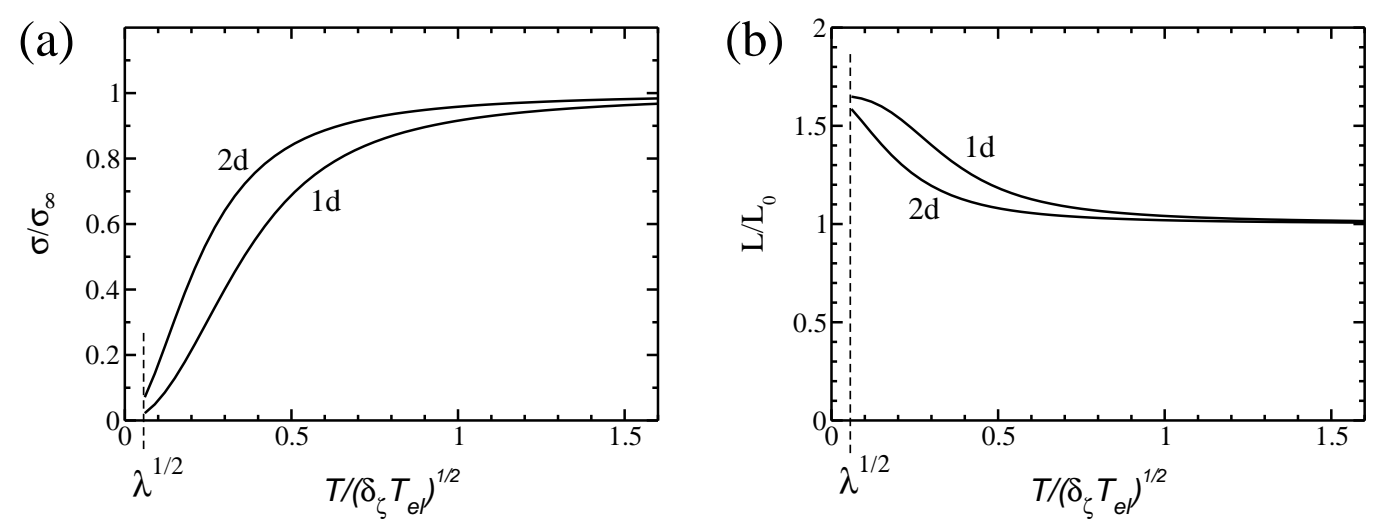

Fig. 10. (a) Lower and upper curves represent the conductivity $\sigma$ in the units of the Drude conductivity $\sigma_{\infty}$ for the $1 \mathrm{~d}$ and $2 \mathrm{~d}$ cases, respectively $\left[\right.$ i.e., $\left\langle\mathcal{B}_{\sigma}^{-1}\right\rangle^{-1}$ and $\left.\exp \left(\left\langle\ln \mathcal{B}_{\sigma}\right\rangle\right)\right]$ as a function of $\sqrt{2 d r(T)}$ - temperature in the units of $\sqrt{\delta_{\zeta} T_{e l}}$, as given by Eqs. (93), (94) in the fast energy relaxation appproximation (this curve does not depend on any parameters). (b) The relative Lorentz number $\mathrm{L} / \mathrm{L}_{0}$ versus temperature in the units of $\sqrt{\delta_{\zeta} T_{e l}}$ for $1 \mathrm{~d}$ (upper curve) and $2 \mathrm{~d}$ (lower curve) cases. Vertical dashed lines indicate the limit of the applicablity of the theory to the metallic region, see Eq. (80).

of the low energy part is less important, and the best tunnelling pairs are different from those for the conductivity. It leads to the renormalization of the Lorentz number (100) by the factor of the order of unity.

We conclude this section by noticing that the contribution of the rare pinholes leads to the deviation from the "natural" assumption $\sigma \propto \zeta_{l o c}^{2} \Gamma^{(i n)}$, cf. Refs. [25,28]. We think, however, that the relative contribution of such configurations at $T_{i n}<T<T_{e l}$ is a model dependent question, and do not pursue this line further.

\section{Stability of the insulating phase.}

Even though the condition (80) gives the sufficient condition for the metallic state to be stable, the consideration of the previous section does not prove the existence or the stability of the insulating phase, but rather gives the indications of the breakdown of the calculational scheme at $T \lesssim T_{i n}$. To show the existence of the transition, we have to prove that perturbation theory from insulating side is also convergent under certain conditions. This analysis is the subject of the present section.

The notion of the statisitical averaging is somewhat non-standard in the insulating phase and its general aspects are discussed in Sec. 6.1. To demonstrate the stability of the insulating phase we linearize the nonlinear equation (69a) in Sec. 6.2 and obtain an equation with the random coefficients somewhat 
similar to that of Ref. [17]. Further consideration will be based on the statistical analysis of this equation, using the technique described in Sec. 6.3. To begin with, we will investigate the simpler but yet instructive case of the zero-dimensional system $I=0$ to illustrate the general structure and make the connections with Ref. [16], see Sec. 6.4. To complete the study, we generalize the consideration to higher dimensions in Sec. 6.5 and calculate the transition temperature.

\subsection{Averaging procedure}

Equations (69) form an infinite set of coupled non-linear equations whose coefficients are functions of the random energies $\xi_{l}$ and occupation numbers $n_{l}(\epsilon)$. Moreover, even though the equilibrium distribution $n_{l}(\epsilon)=\tanh \frac{\epsilon}{2 T}$ nullifies the collision integral, the time of the relaxation of an arbitrary distribution $n_{l}(\epsilon)$ to the equilibrium one becomes infinite for the insulating phase. Therefore, $n_{l}(\epsilon)=\tanh \frac{\epsilon}{2 T}$ has the meaning of the occupation number averaged over an infinitely long period of time (taken to infinity prior to setting the coupling to the bath equal to zero) and bears no information about the state of the system at a given instant of time.

The basis in the space of many-body states of the system is formed by states represented as Slater determinants built on single-particle states $(l, \boldsymbol{\rho})$. Such Slater determinants correspond to the occupation numbers $n_{l}(\epsilon)= \pm 1$. Thus, the decay rates $\Gamma_{l}$ can be considered for each given set $\left\{n_{l}\right\}$. Because the transition may occur only if the number of terms contributing to $\Gamma_{l}$ is large, we can also perform the statistical average with respect to those occupation numbers. We assume them to be arbitrary with the only constraint being to fix the global energy of the system (microcanonical ensemble). Because the number of excitations involved into the formation of the decay rate is much smaller than the total number of the excitations in the system (which scales proportionally to the volume), averaging over the microcanonical ensemble with the energy $E$ (counted from the ground state) is equivalent to the averging over the canonical ensemble with temperature $T$ such that

$$
E=\frac{\pi^{2}}{6} \nu \mathcal{V} T^{2}
$$

where $\nu$ is the averaged density of states per unit volume and $\mathcal{V}$ is the volume

of the system. We assume $T \gg M \delta_{\zeta}$ and verify later that the transition occurs only at such temperature.

To find the distribution functions of the local and thus strongly fluctuating quantities, we perform the ensemble averaging on the final stage of the calcu- 
lation. Thus, the averaging procedure in this section is defined as

$$
\langle\ldots\rangle=\lim _{N \rightarrow \infty} \int_{-N \delta_{\zeta} / 2}^{N \delta_{\zeta} / 2} \prod_{l=1}^{N} \prod_{\rho} \frac{\mathrm{d} \xi_{l}(\boldsymbol{\rho})}{N \delta_{\zeta}} \sum_{n_{l}(\boldsymbol{\rho})= \pm 1} \frac{\exp \frac{n_{l}(\boldsymbol{\rho}) \xi_{l}(\boldsymbol{\rho})}{2 T}}{2 \cosh \frac{\xi_{l}(\boldsymbol{\rho})}{2 T}} \ldots
$$

where the number of levels $N$ and the domain of integration is in accordance with Eq. (48a). Formula (102) does not take into account the level repulsion, see Eq. (48b). This repulsion will be included later when it is necessary.

Let us notice that the same averaging formula would arise if one considered the probability of the occupation (and not the average occupation) of the levels formed as a result of the arbitrarily weak interaction with equilibrium phonons kept at temeperature $T$. In this respect temperature $T$ in Eq. (102) has a meaning of the experimentally measurable quantity.

Having discussed the issue of how to average, we are prepared to calculate the characteristic function (66) to establish the conditions of the stability of the insulating phase.

\subsection{Linearized ImSCBA equations}

To begin the actual analysis of the insulating phase, we notice that in the absence of the external bath $\Gamma_{l}^{(b a t h)}(\epsilon)$, substitution $\Gamma_{l}(\epsilon)=0$ solves the selfconsistency equation (69a). However, in accordance with the discussion of Sec. 4.2 the order of limits is important, so that we have to investigate the stability of the solution $\Gamma_{l}(\epsilon)=0$ with respect to small but finite coupling to the bath. The smallness of $\Gamma_{l}(\epsilon) \propto b$ enables us to linearize the spectral density in Eq. (69a) as

$$
A_{l}(\epsilon) \approx \delta\left(\epsilon-\xi_{l}\right)+\frac{1}{\pi} \frac{\Gamma_{l}(\epsilon)}{\left(\epsilon-\xi_{l}\right)^{2}}
$$

where the second term is understood as the principal value

$$
\frac{1}{\left(\epsilon-\xi_{l}\right)^{2}} \rightarrow \operatorname{Re} \frac{1}{\left(\epsilon-\xi_{l}+i 0\right)^{2}}
$$

We substitute Eq. (103) into the equation for $\Gamma_{l}(\epsilon)$, see Eq. (69a), notice that the probablity of matching the levels into exact resonance equals to zero, and keep the terms only linear in $\Gamma_{l}(\epsilon)$. Taking into account that, according to 
Sec. $6.1, n_{l}(\epsilon)=n_{l}= \pm 1$, we obtain

$$
\begin{aligned}
\Gamma_{l}(\epsilon)= & \Gamma_{l}^{(\text {bath })}(\epsilon)+\sum_{l_{1}, \boldsymbol{a}} \frac{I^{2} \delta_{\zeta}^{2} \Gamma_{l}(\epsilon, \boldsymbol{\rho}+\boldsymbol{a}) \theta_{\Delta}\left[\epsilon-\xi_{l_{1}}(\boldsymbol{\rho}+\boldsymbol{a})\right]}{\left[\epsilon-\xi_{l_{1}}(\boldsymbol{\rho}+\boldsymbol{a})\right]^{2}} \\
& +\sum_{l_{1}, l_{2}, l_{3}} \frac{\lambda^{2} \delta_{\zeta}^{2} Y_{l_{1}, l_{2}}^{l_{3}, l} F_{l_{1}, l_{2} ; l_{3}}^{\Rightarrow} \theta_{\Delta}\left(\epsilon-\xi_{l_{1}}-\xi_{l_{2}}+\xi_{l_{3}}\right)}{\left(\epsilon-\xi_{l_{1}}-\xi_{l_{2}}+\xi_{l_{3}}\right)^{2}} \\
& \times\left[2 \Gamma_{l_{1}}\left(\epsilon-\xi_{l_{2}}+\xi_{l_{3}}\right)+\Gamma_{l_{3}}\left(\xi_{l_{1}}+\xi_{l_{2}}-\epsilon\right)\right],
\end{aligned}
$$

where the denominators are defined in the sense of Eq. (104), and

$$
F_{l_{1}, l_{2} ; l_{3}}^{\Rightarrow}=\frac{1}{4}\left\{1+n_{l_{1}} n_{l_{2}}-n_{l_{3}}\left[n_{l_{1}}+n_{l_{2}}\right]\right\}=\left\{\begin{array}{lc}
1 ; \text { if } & n_{l_{1}}=n_{l_{2}}=-n_{l_{3}}= \pm 1 \\
0 ; & \text { otherwise }
\end{array}\right.
$$

The decay due to the connection with the bath

$$
\Gamma_{l}^{(b a t h)}(\epsilon)=\frac{\left(\epsilon-\xi_{l}\right) b\left(\epsilon-\xi_{l}\right)}{2}\left[\operatorname{coth} \frac{\left(\epsilon-\xi_{l}\right)}{2 T}+n_{l}\right]
$$

is a smooth function of $\epsilon$. As before, the coordinate $\boldsymbol{\rho}$ is assumed to be the same in all terms in the equations unless it is specified explicitly otherwise.

To deal with certain superfluous logarithmic divergences in the future analysis of Eq. (105a), we introduced an ultraviolet cut-off with the help of the function

$$
\theta_{\Delta}(x)=\left\{\begin{array}{l}
1 ;|x| \leq \Delta \\
0 ;|x|>\Delta
\end{array}\right.
$$

This ultraviolet cut-off is introduced for technical convenience only and $\delta_{\zeta} \ll$ $\Delta \lesssim M \delta_{\zeta}$, compare with Eq. (86). The contribution from the part excluded by cut-off does not contain small denominators. This part is self-averaging, proportional to $b(\omega)$, and, thus, it is not relevant for the question of the stability of the insulator, see Eq. (65).

The second term in Eq. (105a) is the effect of the one particle tunneling into the neighboring localization cells. Due to the condition (48f) this term alone does not lead to any significant effects. As this term corresponds to the solution of the one-particle Schrödinger equation, it does not depend on the occupation numbers $n_{l}$. On the other hand, the last term in Eq. (105a) describes the decay due to the excitation of electron-hole pairs. The availability of orbitals for such a process is controlled by the set of $n_{l}= \pm 1$.

Three following subsections are devoted to the statistical analysis of Eq. (105a) with respect to the distribution (102). Namely, we formally solve Eq. (105a) 
as an expansion in small parameters $\lambda^{2}, I^{2}$ :

$$
\Gamma_{l}(\epsilon)=\sum_{k, m} \Gamma_{l}^{(k, m)}(\epsilon) ; \quad \Gamma_{l}^{(0,0)}(\epsilon) \equiv \Gamma_{l}^{b a t h}(\epsilon) ; \quad \Gamma_{l}^{(k, m)}(\epsilon) \propto \lambda^{2 k} I^{2 m}
$$

and calculate the characteristic functions

$$
W^{(k, m)}(s)=\left\langle\exp \left(-s \Gamma_{l}^{(k, m)}(\epsilon)\right)\right\rangle
$$

for each term. The insulator is stable, see condition (66), if the typical value of each term decreases, i.e

$$
\lim _{b \rightarrow 0} \lim _{\mathcal{V} \rightarrow \infty} \lim _{k, m \rightarrow \infty} W^{(k, m)}(s)=1
$$

for any fixed $s>0$.

\subsection{Mayer-Mayer cluster expansion for the characteristic functions}

In the actual calculation of the characteristic function (108) we will use the analogy of this function and averaging procedure (102) with the partition function of the system of classical particles interacting through a certain manyparticle potential. The energy $\xi_{l}$ plays the role of the coordinate, occupation number $n_{l}= \pm 1$ is equivalent to the particle spin, and parameter $s$ is analogous to the "inverse temperature". In order to find these interaction potentials we introduce the diagrammatic representation of Eq. (105a). This representation and all of the notation are shown on Fig. 11.

It is obvious that $k+m$ iterations of the diagrammatic equation shown on Fig. 11 produce diagrams for each term $\Gamma^{(k, m)}$ of Eq. (107). Every term depends on all the orbital energies and all the occupation numbers. At the same time, it can be represented as a sum of terms depending only on the set $\left\{\xi_{l}, n_{l}\right\}_{l=1}^{3 k+m}$, such that

$$
\Gamma^{(k, m)}=\sum_{l_{1}, l_{2}, \ldots, l_{3 k+m}} U\left(\xi_{l_{1}}, n_{l_{1}} ; \ldots ; \xi_{l_{3 k+m}}, n_{l_{3 k+m}}\right),
$$

where we omitted the spatial coordinate $\boldsymbol{\rho}$. Thus, averaging (102) in the expression (108) is indeed equivalent to the calculation of the partition function of $N \rightarrow \infty$ particles interacting via $(3 k+m)$-particle potential $U$.

To perform this calculation we employ the procedure known as Mayer-Mayer cluster expansion [29]:

$$
\ln W^{(k, m)}=\sum_{p=3 k+m}^{\infty} \ln W_{p}^{(k, m)},
$$




$$
\begin{aligned}
& \stackrel{\epsilon}{l}_{l}^{\epsilon}=\stackrel{\epsilon}{\epsilon}_{l}^{\epsilon}+\frac{\epsilon}{t} \frac{\epsilon}{l_{1}, \boldsymbol{\rho}+\boldsymbol{a}} \\
& +2 \stackrel{\epsilon}{\longrightarrow} \underset{l}{l_{l_{3}}}+\stackrel{\xi_{l_{2}}}{\xi_{l_{1}}+\xi_{l_{2}}-\epsilon} \\
& \sum_{l}^{\epsilon}=\Gamma_{l}(\epsilon) \\
& \gtrless_{l}^{\epsilon} \bigcirc=\Gamma_{l}^{(\text {bath })}(\epsilon) \\
& \frac{\epsilon}{l}=\frac{\theta_{\Delta}\left(\epsilon-\xi_{l}\right)}{\left(\epsilon-\xi_{l}\right)^{2}} \\
& \leftarrow+\longleftarrow=1 \\
& =I^{2} \delta_{\zeta}^{2}
\end{aligned}
$$

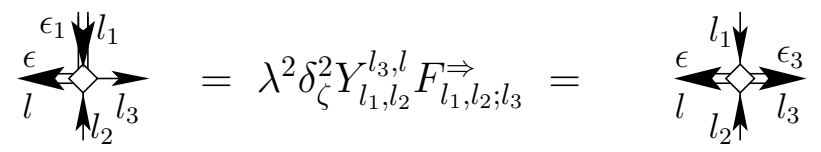

$$
\begin{aligned}
& \epsilon+\xi_{l_{3}}=\epsilon_{1}+\xi_{l_{2}} \quad \epsilon+\epsilon_{3}=\xi_{l_{1}}+\xi_{l_{2}}
\end{aligned}
$$

Fig. 11. Diagrammatic representation of Eq. (105a). Solid lines bear an orbital energy $\xi_{l}$ and an occupation number $n_{l}= \pm 1$. Double lines bear an orbital energy $\xi_{l}$, an occupation number $n_{l}= \pm 1$, and an energy $\epsilon$. The energy carried by the double line is conserved in each hopping vertex (bold rectangle) and changes in the interaction vertex (white square). The algebraic sum of the energies entering the interaction vertex through the double lines and the orbital energies entering through the solid lines is conserved (signs are determined by arrow directions). All the other rules are defined on the figure, and $\theta_{\Delta}$ is defined in Eq. (106).

where the summation is performed over linked clusters of $p$ particles. Analogously to the calculation of the partition function of a classical gas interacting via a two-particle potential $U\left(\boldsymbol{r}_{1}-\boldsymbol{r}_{2}\right)$, whose leading term of the cluster expansion is given by

$$
\ln W_{2}(T)=\int \frac{\mathrm{d}^{3} \boldsymbol{r}}{V}\left[\mathrm{e}^{-\frac{U(\boldsymbol{r})}{T}}-1\right],
$$

for each diagram (110) we introduce the cluster function $f=\mathrm{e}^{-s U}-1$, as shown in Fig. 12. On the same figure we show the diagrammatic representation of the averaging procedure and linking the clusters.

This procedure is especially suitable for the present problem as it systematically takes into account contributions from regions of the phase space where 
(a)

$$
\begin{aligned}
U\left(\xi_{l_{1}}, n_{l_{1}} ; \ldots ; \xi_{l_{p}}, n_{l_{p}}\right) & \equiv f_{s}\left(\xi_{l_{1}}, n_{l_{1}} ; \ldots ; \xi_{l_{p}}, n_{l_{p}}\right) \\
& =e^{-s N_{U} U\left(\xi_{l_{1}}, n_{l_{1}} ; \ldots ; \xi_{l_{p}}, n_{l_{p}}\right)}-1
\end{aligned}
$$

(b)

$$
\begin{array}{ll}
f_{s}\left(\ldots ; \frac{\xi_{l}, n_{l}}{i} ; \ldots\right) & =\int \frac{\mathrm{d} \xi_{l}}{\delta_{\zeta}} \sum_{n_{l}= \pm 1} \frac{e^{\frac{n_{l} \xi_{l}}{2 T}}}{2 \cosh \frac{\xi_{l}}{2 T}} \\
& \times f_{s}\left(\ldots ; \xi_{l}, n_{l} ; \ldots\right)
\end{array}
$$

$$
\begin{aligned}
& f_{s}\left(\ldots ; \frac{\xi_{l}, n_{l}}{\vdots} \underset{\mathfrak{x}}{\mathfrak{x}}, \ldots\right)=\int \frac{\mathrm{d} \xi_{l}}{\delta_{\zeta}} \sum_{n_{l}= \pm 1} \frac{e^{\frac{n_{l} \xi_{l}}{2 T}}}{2 \cosh \frac{\xi_{l}}{2 T}} \\
& \begin{array}{ll}
f_{s}^{\prime}\left(\ldots ; \frac{!}{\xi_{l}, n_{l}} ; \ldots\right) & \times f_{s}\left(\ldots ; \xi_{l}, n_{l} ; \ldots\right) \\
& \times f_{s}^{\prime}\left(\ldots ; \xi_{l}, n_{l} ; \ldots\right)
\end{array}
\end{aligned}
$$

Fig. 12. (a) Definition of the cluster function $f ; N_{U}$ is the degeneracy of the diagram - the number of line permutations keeping the diagram intact. (b) Independent averaging over the position $\xi_{l}$ and the occupation $n_{l}$ of a level $l$. (c) Connected average of two different cluster functions $f$ and $f^{\prime}$ (an arbitrary number of cluster functions can be averaged analogously). The contribution of each linked cluster to $\ln W_{p}^{(k, m)}$ ( $p$ being the number of levels averaged over) should be divided by the number of symmetries of the averaged diagram. This coefficient will be written explicitly in each specific case.

the interaction potentials $U$ assume large values, i.e., of small resonant denominators responsible for delocalization. The next two subsections are dedicated to the application of this procedure.

\subsection{Statistical analysis for zero-dimensional system.}

In this section we consider only transitions inside one localization cell, $m=0$, or, in other words, $I \rightarrow 0$. It suffices for our purposes to limit ourselves with

$$
\left|\epsilon-\xi_{l}\right| \ll M \delta_{\zeta}
$$

To understand the most crucial features of the cluster expansion, we will consider a few lowest order terms explicitly, and then analyze an arbitrary 
order term. It will be useful for us to introduce the notation

$$
\Xi_{l_{1}, \ldots, l_{N_{e}}}^{m_{1}, \ldots, m_{N_{h}}}=\sum_{i=1}^{N_{e}} \xi_{l_{i}}-\sum_{j=1}^{N_{h}} \xi_{m_{j}}
$$

for the energy of an excitation consisting of $N_{e}$ electrons and $N_{h}$ holes.

The lowest order terms are ${ }^{14}$

$$
\begin{aligned}
& \Gamma^{(0,0)}=T b(0) ; \\
& \Gamma^{(1,0)}=\lambda^{2} \delta_{\zeta}^{2} \Gamma^{(0,0)} \sum_{l_{1}, l_{2}, l_{3}} F_{l_{1}, l_{2} ; l_{3}}^{\Rightarrow}\left[\frac{2 Y_{l_{1}, l_{2}}^{l_{3}, l} \theta_{\Delta}\left(\epsilon-\Xi_{l_{1} l_{2}}^{l_{3}}\right)}{\left(\epsilon-\Xi_{l_{1} l_{2}}^{l_{3}}\right)^{2}}+\frac{Y_{l_{1}, l_{2}}^{l_{3}, l} \theta_{\Delta}\left(\epsilon-\Xi_{l_{1} l_{2}}^{l_{3}}\right)}{\left(\epsilon-\Xi_{l_{1} l_{2}}^{l_{3}}\right)^{2}}\right] ;
\end{aligned}
$$

where $\theta_{\Delta}$ is given by Eq. (106). In Eq. (114b), we chose not to join the electron decay and the hole decay even though they are equal to each other. This is done to keep the structure of the subsequent terms more transparent. Moreover, this form will be useful for the discussion of the modification of ImSCBA in Sec. 7.3.

Let us use the machinery of Sec. 6.3 to find the characteristic function of the rate $(114 \mathrm{~b})$. The corresponding three-particle potentials are given by

$$
U_{12 ; 3}^{e}=2 U_{12 ; 3}^{h}=\frac{2 \Gamma^{(0,0)} \lambda^{2} \delta_{\zeta}^{2} Y_{1,2}^{3, l} F^{\Rightarrow}\left(n_{1}, n_{2}, n_{3}\right)}{\left(\epsilon-\Xi_{12}^{3}\right)^{2}}
$$

switched on for certain combinations $\left\{n_{1}, n_{2}, n_{3}\right\}$, see Eq. (105b). The cutoff $\theta_{\Delta}\left(\epsilon-\Xi_{12}^{3}\right)$ will be incorporated in the definition of cluster function $f$ below.

The main conribution comes from clusters with $p=3$ particles, see Fig. 13a:

$$
\begin{aligned}
& \ln W_{3}^{(1,0)}=\prod_{l=1}^{3}\left(\int \frac{\mathrm{d} \xi_{l}}{\delta_{\zeta}} \sum_{n_{l}= \pm 1} \frac{\exp \frac{n_{l} \xi_{l}}{2 T}}{2 \cosh \frac{\xi_{l}}{2 T}}\right) \tilde{f}_{12 ; 3} ; \\
& \tilde{f}_{12 ; 3}=f_{12 ; 3}^{e}+\frac{1}{2} f_{12 ; 3}^{h}+\frac{1}{2} f_{12 ; 3}^{e}\left\{f_{21 ; 3}^{e}+f_{12 ; 3}^{h}\left[2+f_{21 ; 3}^{e}\right]\right\} \\
& f_{12 ; 3}^{e}=\left(\mathrm{e}^{-s U_{12 ; 3}^{e}}-1\right) \theta_{\Delta}\left(\epsilon-\Xi_{12}^{3}\right) \text {, } \\
& f_{12 ; 3}^{h}=\left(\mathrm{e}^{-2 s U_{12 ; 3}^{h}}-1\right) \theta_{\Delta}\left(\epsilon-\Xi_{12}^{3}\right),
\end{aligned}
$$

\footnotetext{
${ }^{14}$ We assume that $b(\omega=0)>0$. This assumption does not correspond to any physical phonons. Actual frequency dependence $b(\omega) \propto \omega^{n}$ is important if one tries to calculate the physical conductivity in the insulating regime. It is however not important for the determination of the stability of the inslulating phase.
} 
(a) $\ln W_{3}^{(1,0)}=$
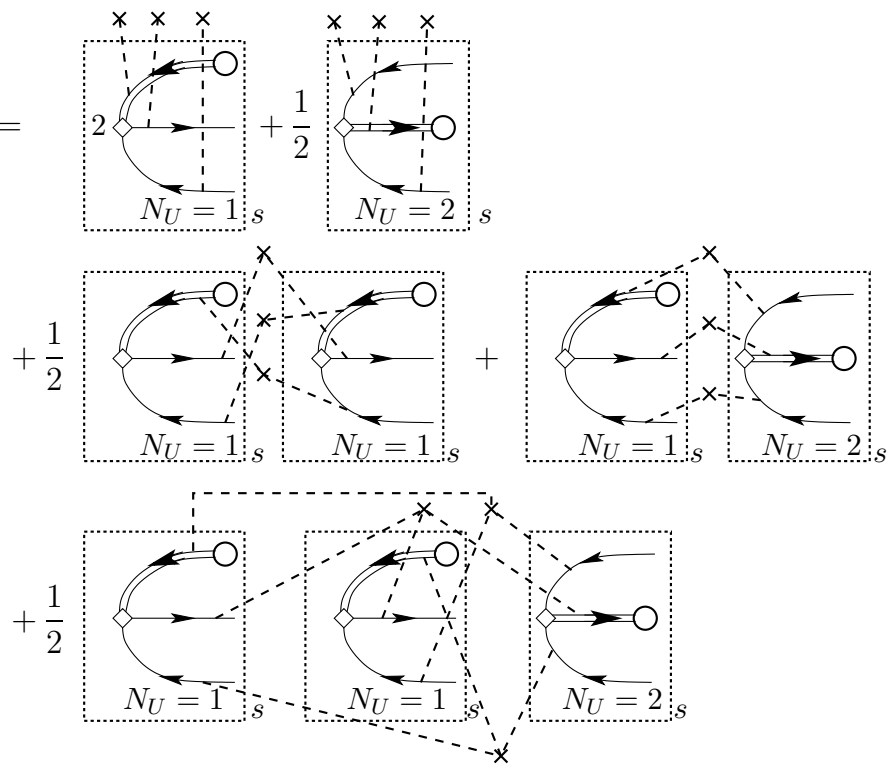

(b)

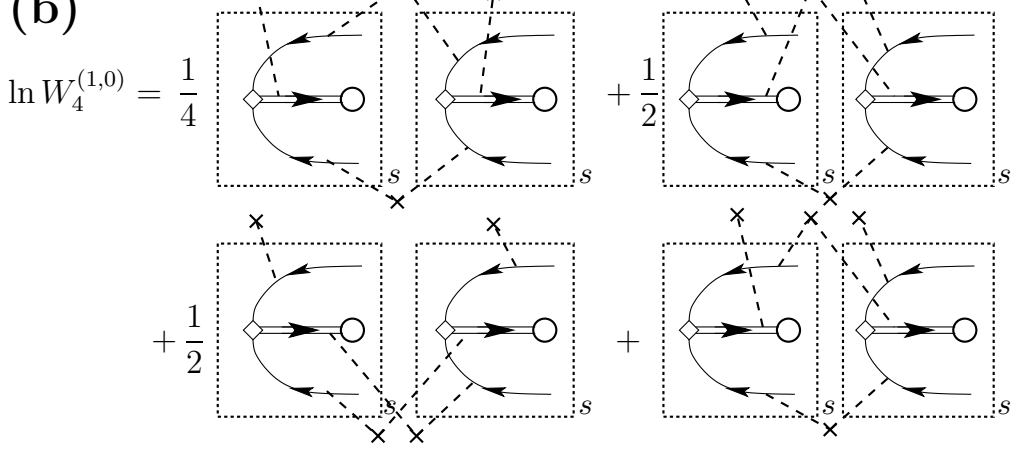

(c)
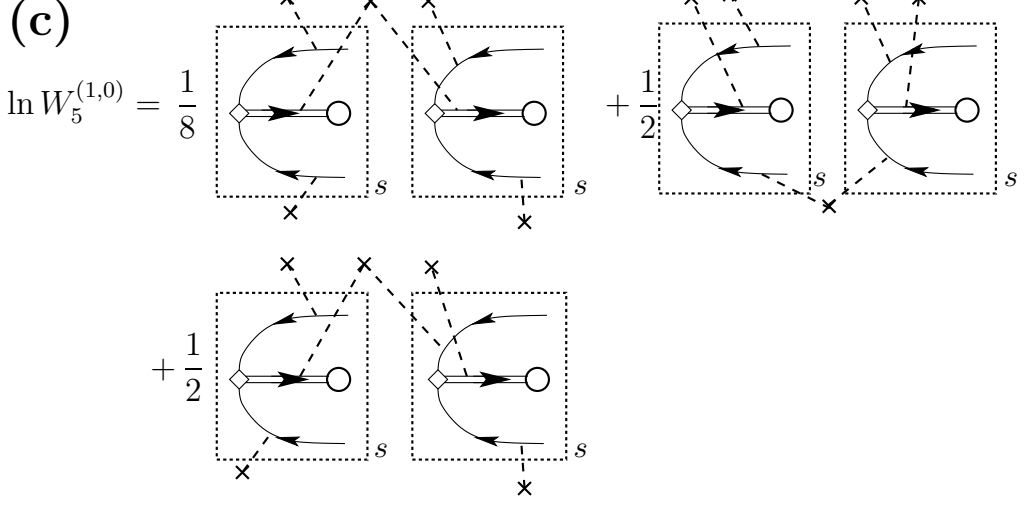

Fig. 13. The 3-, 4-, and 5-particle contributions to $\ln W^{(1,0)}$. For three particles (a), the cross-correlations between the electron and the hole contributions, the last term in Eq. (116b), are shown in the second and third lines. For 4 and 5 particles $(b, c)$ only the hole-hole terms are shown. When not displayed, the degeneracy for all diagrams $N_{U}$ are the same as on panel (a). 
where the coefficient $1 / 2$ and extra factor of $N_{U}=2$ in the exponential in $f_{12 ; 3}^{h}$ take care of the symmetry $f_{12 ; 3}^{h}=f_{21 ; 3}^{h}$, second term on Fig. 13a. The last termm in Eq. (116b) describes the cross-correlations between the electron and the hole contributions as the potentials $U^{e}\left(\xi_{1}, \xi_{2}, \xi_{3}\right), U^{e}\left(\xi_{2}, \xi_{1}, \xi_{3}\right)$, and $U^{h}\left(\xi_{1}, \xi_{2}, \xi_{3}\right)$ diverge for the same sets of $\xi_{1}, \xi_{2}, \xi_{3} .{ }^{15}$

Performing the integration with the help of Eq. (105b) and definition of $Y$, see Eq. (69a), we obtain using the condition (112):

$$
\ln W_{3}^{(1,0)}=-\frac{1}{\sqrt{3}}\left(\pi s \Gamma^{(0,0)}\right)^{1 / 2} \frac{3 \lambda T}{\delta_{\zeta}} \mathcal{M}\left(\frac{2 T}{\delta_{\zeta}}, \frac{\epsilon}{2 T}\right),
$$

where the numerical coefficient $1 / \sqrt{3}$ appears due to the cross-correlation terms in Eq. (116b).

Parameter $T \mathcal{M}_{d}\left(\frac{2 T}{\delta_{\zeta}}, \frac{\epsilon}{2 T}\right)$ roughly corresponds to the total energy of all electronhole pairs participating in the process of the decay of a quasiparticle with the energy $\epsilon$. The explicit expression for $\mathcal{M}_{d}$ is

$$
\mathcal{M}(y, z)=\frac{y}{2} \int \frac{\mathrm{d} x_{1} \mathrm{~d} x_{2}\left|\Upsilon^{2}\left(y x_{1}\right)-\Upsilon^{2}\left(y x_{2}\right)\right| \cosh z}{\cosh \left(x_{1}+x_{2}+z\right) \prod_{i=1,2} \cosh \left(x_{i}+z\right)}
$$

Performing integration in Eq. (118) with the help of Eq. (48h), we obtain for $T \gg M \delta_{\zeta}$

$$
\mathcal{M}=2 M
$$

independently of $T$ and $\epsilon$. This occurs because of the additional restriction on the phase volume for the decay of one-particle excitation into the three-particle excitations by the energy dependence of the matrix elements.

Although the metal-insulator transition is a property of large orders of perturbation theory, see Eq. (109), its precursor can be seen already in the characteristic function of the lowest order term (117). Indeed, condition $\ln W^{(1,0)}\left(s^{*}\right) \simeq$ 1 gives the most probable value $\Gamma_{t y p}^{(1,0)} \simeq 1 / s^{*}$ of the distribution. Requiring $\Gamma_{\text {typ }}^{(1,0)}<\Gamma^{(0,0)}$, we obtain the condition $T<T_{*}$, where

$$
\left(\frac{\lambda M T_{*}}{\delta_{\zeta}}\right) \simeq 1
$$

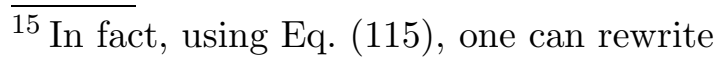

$$
\tilde{f}_{12 ; 3}=\frac{1}{2}\left(1+f_{12 ; 3}^{e}\right)\left(1+f_{21 ; 3}^{e}\right)\left(1+f_{12 ; 3}^{h}\right)=\frac{1}{2}\left[\exp \left(-6 U^{h}\right)-1\right]
$$

i.e., join all the term in one cluster function. This joining, however, would obscure the structure of the higher order terms and we choose not to perform this operation. 
i.e., $T_{*}$ is roughly of the order of the temperature $T_{i n}$ limiting the stability of the metallic phase, see Eq. (80). Consideration of higher orders of the perturbation theory to be performed shortly will only slightly refine expression (120), see Eq. (131).

To complete the analysis of the lowest order term (114), we verify the validity of the cluster expansion, which amounts to finding the condition for which $\left|\ln W_{3}^{(1,0)}(s)\right| \gg\left|\ln W_{4,5}^{(1,0)}(s)\right|$. Diagrams for $\ln W_{4,5}^{(1,0)}(s)$ are shown in Fig. 13b,c. They give

$$
\begin{aligned}
\ln W_{4}^{(1,0)}(s)= & \prod_{l=1}^{4}\left(\int \frac{\mathrm{d} \xi_{l}}{\delta_{\zeta}} \sum_{n_{l}= \pm 1} \frac{\exp \frac{n_{l} \xi_{l}}{2 T}}{2 \cosh \frac{\xi_{l}}{2 T}}\right) \\
& \times \frac{1}{4}\left(\tilde{f}_{12 ; 3} \tilde{f}_{12 ; 4}+2 \tilde{f}_{13 ; 2} \tilde{f}_{14 ; 2}+2 \tilde{f}_{13 ; 2} \tilde{f}_{24 ; 1}+4 \tilde{f}_{12 ; 3} \tilde{f}_{14 ; 2}\right) \\
\ln W_{5}^{(1,0)}(s)= & \prod_{l=1}^{5}\left(\int \frac{\mathrm{d} \xi_{l}}{\delta_{\zeta}} \sum_{n_{l}= \pm 1} \frac{\exp \frac{n_{l} \xi_{l}}{2 T}}{2 \cosh \frac{\xi_{l}}{2 T}}\right) \\
& \times \frac{1}{8}\left(\tilde{f}_{12 ; 5} \tilde{f}_{34 ; 5}+4 \tilde{f}_{51 ; 2} \tilde{f}_{53 ; 4}+4 \tilde{f}_{12 ; 5} \tilde{f}_{53 ; 4}\right)
\end{aligned}
$$

Using $\tilde{f}_{12,3}$ from Eq. (116b), we can estimate these expressions as

$$
\ln W_{4}^{(1,0)}(s) \simeq s \Gamma^{(0,0)} \frac{\lambda^{2} M T}{\delta_{\zeta}}, \quad \ln W_{5}^{(1,0)}(s) \simeq s \Gamma^{(0,0)} \frac{\lambda^{2} M T^{2}}{\delta_{\zeta}^{2}}
$$

where $M$ is defined in Eq. (48h). Comparing Eq. (122) with Eq. (117), we find that for $T \gg \delta_{\zeta}$ the cluster expansion is justified even for $\left|\ln W_{3}^{(1,0)}\right| \gg 1$. The origin of this suppression of the larger clusters is the same as the one controlling the virial expansion for the classical gases - clusters involving more particles impose more restrictions on the phase volume.

Our next task is to consider an arbitrary order term $\Gamma^{(k, 0)}$. To explore how the lowest-order result Eq. (117) is modified, we consider $\Gamma^{(2,0)}$ first. Performing 


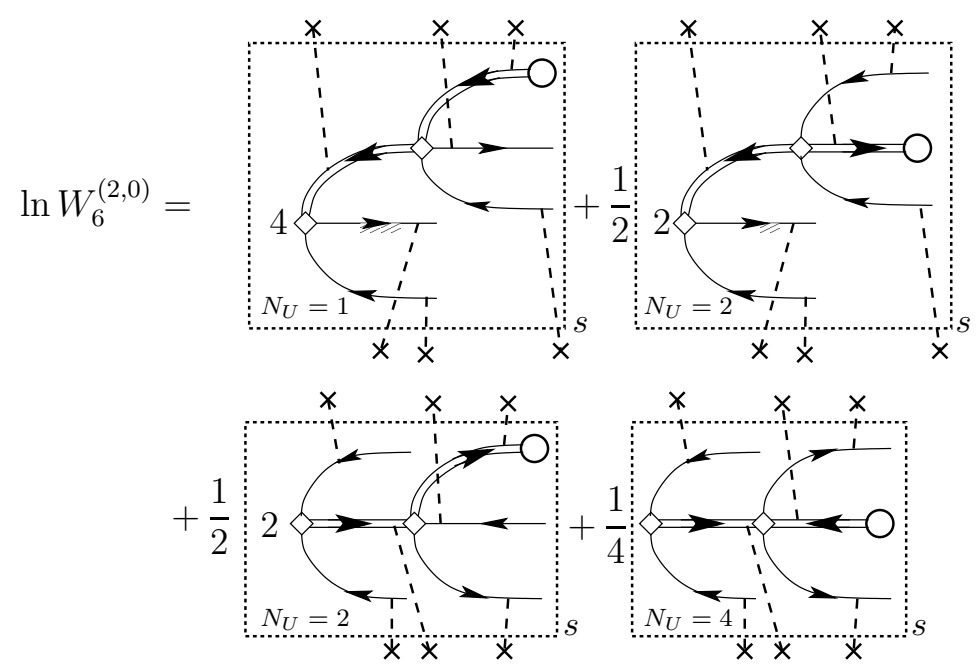

Fig. 14. The leading contributions to the cluster expansion of $\ln W^{(2,0)}$. The crosscorrelations between the electron and the hole contributions, second and third lines in Eq. (125b), are not shown.

one more iteration in Eq. (105a) with the help of Eq. (114), we find

$$
\begin{aligned}
& \Gamma^{(2,0)}=\Gamma^{(0,0)} \lambda^{4} \delta_{\zeta}^{4} \sum_{l_{1}, l_{2} ; l_{3}} F_{l_{1}, l_{2} ; l_{3}}^{\Rightarrow} \sum_{l_{4}, l_{5} ; l_{6}}{ }^{\prime} F_{l_{4}, l_{5} ; l_{6}}^{\Rightarrow} \\
& \times\left\{\frac{2 Y_{l_{1}, l_{2}}^{l_{3}, l} \theta_{\Delta}\left(\epsilon-\Xi_{l_{1} l_{2}}^{l_{3}}\right)}{\left(\epsilon-\Xi_{l_{1} l_{2}}^{l_{3}}\right)^{2}} \frac{2 Y_{l_{4}, l_{5}}^{l_{1}, l_{6}} \theta_{\Delta}\left(\epsilon-\Xi_{l_{2} l_{4} l_{5}}^{l_{3} l_{6}}\right)}{\left(\epsilon-\Xi_{l_{2} l_{4} l_{5}}^{l_{3} l_{6}}\right)^{2}}\right. \\
& +\frac{2 Y_{l_{1}, l_{2}}^{l_{3}, l} \theta_{\Delta}\left(\epsilon-\Xi_{l_{1} l_{2}}^{l_{3}}\right)}{\left(\epsilon-\Xi_{l_{1} l_{2}}^{l_{3}}\right)^{2}} \frac{Y_{l_{4}, l_{5}}^{l_{1}, l_{6}} \theta_{\Delta}\left(\epsilon-\Xi_{l_{1} l_{2} l_{6}}^{l_{4} l_{5}}\right)}{\left(\epsilon-\Xi_{l_{2} l_{4} l_{5}}^{l_{3} l_{6}}\right)^{2}} \\
& +\frac{Y_{l_{1}, l_{2}}^{l_{3}, l} \theta_{\Delta}\left(\epsilon-\Xi_{l_{1} l_{2}}^{l_{3}}\right)}{\left(\epsilon-\Xi_{l_{1} l_{2}}^{l_{3}}\right)^{2}} \frac{2 Y_{l_{3}, l_{6}}^{l_{4}, l_{5}} \theta_{\Delta}\left(\epsilon-\Xi_{l_{1} l_{2} l_{6}}^{l_{4} l_{5}}\right)}{\left(\epsilon-\Xi_{l_{1} l_{2} l_{6}}^{l_{4} l_{5}}\right)^{2}} \\
& \left.+\frac{Y_{l_{1}, l_{2}}^{l_{3}, l} \theta_{\Delta}\left(\epsilon-\Xi_{l_{1} l_{2}}^{l_{3}}\right)}{\left(\epsilon-\Xi_{l_{1} l_{2}}^{l_{3}}\right)^{2}} \frac{Y_{l_{3}, l_{6}}^{l_{4}, l_{5}} \theta_{\Delta}\left(\epsilon-\Xi_{l_{1} l_{2} l_{6}}^{l_{4} l_{5}}\right)}{\left(\epsilon-\Xi_{l_{1} l_{2} l_{6}}^{l_{4} l_{5}}\right)^{2}}\right\} \text {, }
\end{aligned}
$$

each term is shown on Fig. 14. Here prime in the second sum excludes the terms with $l_{4}, l_{5}=l_{1}, l_{2}$ and $l_{6}=l_{3}$, see Fig. $7 \mathrm{~b} .2$. (Let us note that the placement of crosses on Fig. 14 automatically takes this exclusion into account.) Similarly to the expression for $\Gamma^{(1,0)}$ in Eq. (114), we chose not to join some similar terms together. 
The corresponding 6-particle potentials are given by

$$
\begin{aligned}
& U^{e e}=2 U^{e h}=\frac{4 \Gamma^{(0,0)} \lambda^{4} \delta_{\zeta}^{4} Y_{1,2}^{3, l} F_{1,2 ; 3}^{\Rightarrow} Y_{4,5}^{6,1} F_{4,5 ; 6}^{\Rightarrow}}{\left(\epsilon-\Xi_{12}^{3}\right)^{2}\left(\epsilon-\Xi_{245}^{36}\right)^{2}} ; \\
& U^{h e}=2 U^{h h}=\frac{2 \Gamma^{(0,0)} \lambda^{4} \delta_{\zeta}^{4} Y_{1,2}^{3, l} F_{1,2 ; 3}^{\Rightarrow} Y_{4,5}^{6,3} F_{4,5 ; 6}^{\Rightarrow}}{\left(\epsilon-\Xi_{12}^{3}\right)^{2}\left(\epsilon-\Xi_{126}^{45}\right)^{2}} .
\end{aligned}
$$

The leading term of the cluster expansion shown on Fig. 14, is given by $[c f$. Eq. (15)]

$$
\begin{aligned}
& \ln W^{(2,0)}=\prod_{l=1}^{6}\left(\int \frac{\mathrm{d} \xi_{l}}{\delta_{\zeta}} \sum_{n_{l}= \pm 1} \frac{\exp \frac{n_{l} \xi_{l}}{2 T}}{2 \cosh \frac{\xi_{l}}{2 T}}\right) \tilde{f}\left(\begin{array}{c}
12 ; 3 \\
45 ; 6
\end{array}\right) ; \\
& \tilde{f}\left(\begin{array}{c}
12 ; 3 \\
54 ; 6
\end{array}\right)=f^{e e}\left(\begin{array}{c}
12 ; 3 \\
45 ; 6
\end{array}\right)+\frac{1}{2} f^{e h}\left(\begin{array}{c}
12 ; 3 \\
45 ; 6
\end{array}\right)+\frac{1}{2} f^{h e}\left(\begin{array}{c}
12 ; 3 \\
45 ; 6
\end{array}\right)+\frac{1}{4} f^{h h}\left(\begin{array}{c}
12 ; 3 \\
45 ; 6
\end{array}\right) \\
& +\frac{1}{2} f^{e e}\left(\begin{array}{c}
12 ; 3 \\
45 ; 6
\end{array}\right)\left\{f^{e e}\left(\begin{array}{l}
12 ; 3 \\
54 ; 6
\end{array}\right)+f^{e h}\left(\begin{array}{c}
12 ; 3 \\
45 ; 6
\end{array}\right)\left[2+f^{e e}\left(\begin{array}{c}
12 ; 3 \\
54 ; 6
\end{array}\right)\right]\right\} \\
& +\frac{1}{4} f^{h e}\left(\begin{array}{l}
12 ; 3 \\
45 ; 6
\end{array}\right)\left\{f^{h e}\left(\begin{array}{c}
12 ; 3 \\
54 ; 6
\end{array}\right)+f^{h h}\left(\begin{array}{c}
12 ; 3 \\
45 ; 6
\end{array}\right)\left[2+f^{h e}\left(\begin{array}{c}
12 ; 3 \\
54 ; 6
\end{array}\right)\right]\right\} \text {; } \\
& f^{e e}\left(\begin{array}{c}
12 ; 3 \\
54 ; 6
\end{array}\right)=\left[\mathrm{e}^{-s U^{e e}\left(\begin{array}{c}
12 ; 3 \\
54 ; 6
\end{array}\right)}-1\right] \theta_{\Delta}\left(\epsilon-\Xi_{12}^{3}\right) \theta_{\Delta}\left(\epsilon-\Xi_{245}^{36}\right) \text {; } \\
& f^{e h}\left(\begin{array}{c}
12 ; 3 \\
54 ; 6
\end{array}\right)=\left[\mathrm{e}^{-2 s U^{e h}\left(\begin{array}{c}
12 ; 3 \\
54 ; 6
\end{array}\right)}-1\right] \theta_{\Delta}\left(\epsilon-\Xi_{12}^{3}\right) \theta_{\Delta}\left(\epsilon-\Xi_{245}^{36}\right) \text {; } \\
& f^{h e}\left(\begin{array}{c}
12 ; 3 \\
54 ; 6
\end{array}\right)=\left[\mathrm{e}^{-2 s U^{h e}\left(\begin{array}{c}
12 ; 3 \\
54 ; 6
\end{array}\right)}-1\right] \theta_{\Delta}\left(\epsilon-\Xi_{12}^{3}\right) \theta_{\Delta}\left(\epsilon-\Xi_{126}^{45}\right) \text {; } \\
& f^{h h}\left(\begin{array}{c}
12 ; 3 \\
54 ; 6
\end{array}\right)=\left[\mathrm{e}^{-4 s U^{h h}\left(\begin{array}{c}
12 ; 3 \\
54 ; 6
\end{array}\right)}-1\right] \theta_{\Delta}\left(\epsilon-\Xi_{12}^{3}\right) \theta_{\Delta}\left(\epsilon-\Xi_{126}^{45}\right) \text {. }
\end{aligned}
$$

Similarly to Eq. (116b), the second and the third lines in Eq. (125b) express the cross-correlation between different terms in the lowest order perturbation theory. These correlations do not proliferate into higher order terms; in particular, $U^{e e}$ from Eq. (124) is not invariant under permutation $1 \leftrightarrow 2$.

Substituting Eq. (124) into Eq. (125) and using the approximate expression for the integral

$$
\begin{aligned}
& \mathcal{Z}_{n}(y, z) \equiv \int_{0}^{z} \mathrm{~d} x_{1} \ldots \mathrm{d} x_{n}\left[\exp \left(-\frac{y}{x_{1}^{2} \ldots x_{n}^{2}}\right)-1\right] \\
& =-\sqrt{\pi y} \frac{\left[\ln z^{n} y^{-1 / 2}+\mathcal{O}(1)\right]^{n-1}}{(n-1) !} ; \quad y \ll\left[\mathrm{e}^{-1} z\right]^{2 n},
\end{aligned}
$$


we find

$$
\ln W^{(2,0)}=-\frac{1}{\sqrt{3}}\left(\pi s \Gamma^{(0,0)}\right)^{1 / 2}\left[\frac{6 \lambda M T}{\delta_{\zeta}}\right]^{2} \times \ln \left(\frac{\Delta^{2}}{\left(s \Gamma^{(0,0)}\right)^{1 / 2}\left(\lambda \delta_{\zeta}\right)^{2}}\right)
$$

where $\Delta$ is the scale introduced in Eq. (106), and the coefficient $1 / \sqrt{3}$ comes from the cross-correlations in Eq. (125b).

The analysis of the corrections to Eq. (127) is performed similarly to the estimate of Eq. (121), see Appendix C. It shows that their main effect is the more accurate determination of the cut-off of the logarithm such that

$$
\Delta \rightarrow \delta_{\zeta}
$$

so that Eq. (127) is valid in a leading logarithmic approximation. This value of the cut-off is in agreement with Ref. [20].

All the higher order terms are considered analogously, and we find

$$
\ln W^{(n, 0)}=-\sqrt{\frac{\pi s \Gamma^{(0,0)}}{3}}\left(\frac{6 \lambda M T}{\delta_{\zeta}}\right)^{n} \frac{1}{(n-1) !} \ln ^{n-1}\left(\frac{\Delta^{n}}{\left(s \Gamma^{(0,0)}\right)^{1 / 2}\left(\lambda \delta_{\zeta}\right)^{n}}\right) .
$$

To investigate metal-insulator transition we have to consider the behavior at $n \rightarrow \infty$. We obtain from Eq. (129) with the logarthimic accuracy:

$$
\ln W^{(n \gg 1,0)} \simeq-\left(s \Gamma^{(0,0)}\right)^{\gamma(T) / 2}\left(\frac{6 \mathrm{e} \lambda M T}{\delta_{\zeta}} \ln \frac{\Delta}{\lambda \delta_{\zeta}}\right)^{n}, \quad \gamma(T)=1-\left[\ln \frac{\Delta}{\lambda \delta_{\zeta}}\right]^{-1} .
$$

Equations (130) constitute the central result of this section as they describe the complete statistics of each term in the ImSCBA series. If many particles are in the excited state ( $T$ is finite), formula (130a) predicts the phase transition. Indeed, the stability criterion (109) is violated at $T>T^{*}$, where

$$
\frac{6 \mathrm{e} \lambda M T_{*}}{\delta_{\zeta}} \ln \frac{1}{\lambda}=1
$$

where we used the replacement (128) in the argument of the logarithm. Equation (131) refines Eq. (120).

This would mean that insulator becomes unstable at $T>T_{*}$. This conclusion, however, is an artefact of the averaging procedure (102) which neglected the one-particle level repulsion, see Eq. (48b). To take this level repulsion into account we use the following qualitative consideration. Inspection of each term 
in the perturbaiton theory, see, e.g., Eq. (15) or (125), shows that $W^{(n, 0)}$ is contributed by $2 n$ integrations over $-T<\xi_{l}<T$. It means that $W^{(n, 0)}$ is essentially determined by the probability to find $2 n$ levels within the interval with the width $T$. According to Eq. (48b), this probability is suppressed by the level repulsion. As a result, Eq. (130a) is modified as

$$
\ln W^{(n \gg 1,0)} \simeq-\left(s \Gamma^{(0,0)}\right)^{\gamma / 2}\left(\frac{6 \mathrm{e} \lambda M T}{\delta_{\zeta}} \ln \frac{\Delta}{\lambda \delta_{\zeta}}\right)^{n} \exp \left[-\mathcal{P}\left(\frac{c n \delta_{\zeta}}{T}\right)\right]
$$

where function $\mathcal{P}$ is defined in Eq. (48b), and $c$ is the numerical factor of the order of unity. Because $\lim _{x \rightarrow \infty} x^{-1} \mathcal{P}(x)=\infty$, we find

$$
\lim _{n \rightarrow \infty} \ln W^{(n, 0)}=0 \quad \text { for any } T \text {. }
$$

Equation (132) describes the impossibility of a genuine phase tranistion in a finite system where the unphysical packing of infinite number of levels in a finite enery strip is forbidden.

Even though for a zero-dimensional system temperature $T_{*}$ does not have a physical meaning of the transition temperature, the finite coupling between the localization cells leads to the phase transition with $T_{c}$ very close to $T_{*}$, as we will show in the next subsection.

\subsection{Statistical analysis and metal-insulator transition in finite-dimensional systems.}

The goal of this subsection is to generalize Eqs. (130) by including hopping into the neighbouring localization cells. As a result, the effect of the finite size (132) will be overcome and the genuine metal-insulator tranistion will occur. Similarly to the previous subsection, we will consider explicitly a few lower orders of the perturbation theory, and then obtain the result for an arbitrary order.

The contribution which involves hopping only (its first two terms $\Gamma^{(0,1)}$ and $\Gamma^{(0,2)}$ are shown in Fig. 15) corresponds to the purely single-particle problem. Since the exact single-particle eigenstates are assumed to be localized, see condition (48f), this contribution does not lead to delocalization. Thus, we start with the term $\Gamma^{(1,1)}$, which involves tunneling of the particle and creation 
of an electron-hole pair. By iterating Eq. (105a) twice, we find (see Fig. 15):

$$
\begin{aligned}
& \Gamma_{l}^{(1,1)}(\boldsymbol{\rho})=\lambda^{2} I^{2} \delta_{\zeta}^{4} \Gamma^{(0,0)} \sum_{l_{1}, \ldots, l_{4} ; \boldsymbol{a}}\left\{\frac{2 Y_{l_{1}^{+}, l_{2}^{+}}^{l_{+}^{+}, l_{l_{1}^{+}}} F_{2}^{{ }_{2}} ;_{3}^{+} \theta_{\Delta}\left(\epsilon-\xi_{l_{4}^{+}}\right) \theta_{\Delta}\left(\epsilon-\Xi_{l_{1}^{+} l_{2}^{+}}^{l_{+}^{+}}\right)}{\left(\epsilon-\xi_{l_{4}^{+}}\right)^{2}\left(\epsilon-\Xi_{l_{1}^{+} l_{2}^{+}}^{l_{+}^{+}}\right)^{2}}\right. \\
& \times\left\{\frac{Y_{l_{1}^{+}, l_{2}^{+}}^{l^{+}, l_{l_{1}^{+}}^{+}} F_{2}^{l_{+} ; l_{3}^{+}} \theta_{\Delta}\left(\epsilon-\xi_{l_{4}^{+}}\right) \theta_{\Delta}\left(\epsilon-\Xi_{l_{1}^{+} l_{2}^{+}}^{l_{+}^{+}}\right)}{\left(\epsilon-\xi_{l_{4}^{+}}\right)^{2}\left(\epsilon-\Xi_{l_{1}^{+} l_{2}^{+}}^{l_{+}^{+}}\right)^{2}}\right. \\
& +\frac{2 Y_{l_{1}, l_{2}}^{l_{3}, l} F_{l_{1}, l_{2} ; l_{3}}^{\Rightarrow} \theta_{\Delta}\left(\epsilon-\Xi_{l_{1} l_{2}}^{l_{3}}\right) \theta_{\Delta}\left(\epsilon-\Xi_{l_{2} l_{4}^{+}}^{l_{3}}\right)}{\left(\epsilon-\Xi_{l_{1} l_{2}}^{l_{3}}\right)^{2}\left(\epsilon-\Xi_{l_{2} l_{4}^{+}}^{l_{3}}\right)^{2}} \\
& \left.+\frac{Y_{l_{1}, l_{2}}^{l_{3}, l} F_{l_{1}, l_{2} ; l_{3}}^{\Rightarrow} \theta_{\Delta}\left(\epsilon-\Xi_{l_{1} l_{2}}^{l_{3}}\right) \theta_{\Delta}\left(\Xi_{l_{2} l_{1}}^{l_{4}^{+}}-\epsilon\right)}{\left(\epsilon-\Xi_{l_{1} l_{2}}^{l_{3}}\right)^{2}\left(\Xi_{l_{2} l_{1}}^{l_{+}^{+}}-\epsilon\right)^{2}}\right\} .
\end{aligned}
$$

where we used the short-hand notation (113), and $\xi_{l} \equiv \xi_{l}(\boldsymbol{\rho}), \xi_{l^{+}} \equiv \xi_{l}(\boldsymbol{\rho}+\boldsymbol{a})$.

From Fig. 15 we find (omitting overall numerical coefficient)

$$
\ln W^{(1,1)} \simeq-\left(s \Gamma^{(0,0)}\right)^{1 / 2}\left(\frac{12 d I \lambda M T}{\delta_{\zeta}}\right) \ln \left[\frac{\Delta^{2}}{\left(s \Gamma^{(0,0)}\right)^{1 / 2} \delta_{\zeta}^{2} I \lambda}\right]
$$

Comparing Eq. (134) with Eq. (127), we see that by replacing the electron-hole pair creation to a single-particle hopping, we always introduce the additional smallness. Thus, we can anticipate that the number of hoppings must be as small as possible to maximize the overall $W^{(m, n)}$ for fixed $m+n$. The only reason to include hopping at all is to overcome the finite number of levels restriction which suppressed the transition in 0-dimensional case of previous subsection.

The result for the arbitrary order perturbation theory may be immediately obtained by examining the differences between Eq. (134) with Eq. (127), and analogy with Eq. (129):

$$
\begin{aligned}
& \ln W^{(n, m)}=-\left(s \Gamma^{(0,0)}\right)^{1 / 2}\left(\frac{6 \lambda M T}{\delta_{\zeta}}\right)^{n} I^{m} \\
& \times \frac{\mathcal{C}(n, m)}{(n+m-1) !} \ln ^{n+m-1}\left[\frac{\left(s \Gamma^{(0,0)}\right)^{-1 / 2} \Delta^{n+m}}{\delta_{\zeta}^{n+m} \lambda^{n} I^{m}}\right] .
\end{aligned}
$$




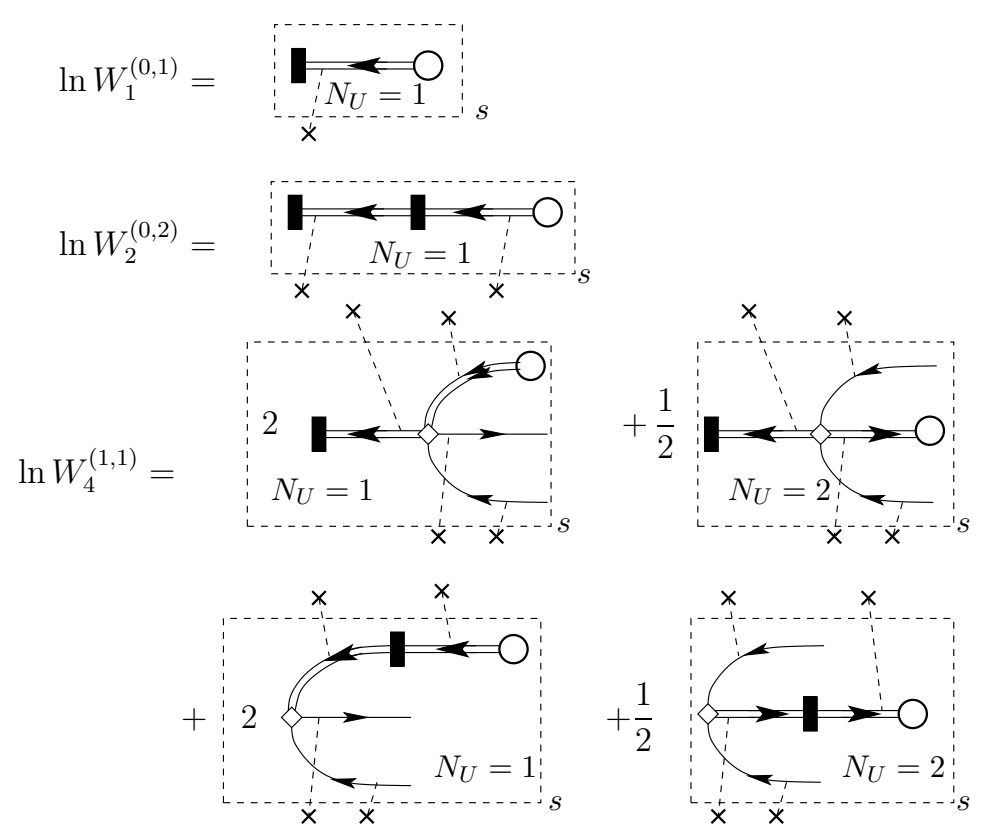

Fig. 15. The leading contributions to $\ln W^{(0,1)}, \ln W^{(0,2)}$, and $\ln W^{(1,1)}$. The cross-correlations between the electron and hole contributions are not shown.

The most important difference with Eq. (129) is the presence of the additional factor $\mathcal{C}(n, m)$ which has the meaning of the number of ways to order the $n$ interactions and $m$ hoppings with respect to each other. The expression for these coefficients is

$$
\mathcal{C}(n, m)=\prod_{i=1}^{m} \sum_{\boldsymbol{a}_{i}} \sum_{N_{i}=0}^{\infty} \delta_{n, \sum_{i} N_{i}} \exp \left[-\sum_{\boldsymbol{\rho}} \mathcal{P}\left(\frac{c \delta_{\zeta} \sum_{i=1}^{m} N_{i} \delta_{\boldsymbol{\rho}, \boldsymbol{\rho}_{0}+\sum_{j=0}^{i} \boldsymbol{a}_{j}}}{T}\right)\right]
$$

where $\boldsymbol{\rho}_{0}$ is an arbitrary site on the lattice, $\boldsymbol{a}$ are the vectors connecting each site to its nearest neighbours. The meaning of the coefficients, $\mathcal{C}(n, m)$, is the total number of random lines consisting of $m$ segments on the $d$-dimensional cubic lattice. Integer $N_{i}$ is the number of electron hole pairs emitted by the electron between the hopping event $i$ and the hopping event $(i+1)$. We included the effect of the level repulsion within each localization cell in the same spirit as in Eq. (132). The argument of $\mathcal{P}$ counts the total number of electronhole pairs within localization site $\boldsymbol{\rho}$ and takes into account the fact that the random path may traverse one site more than once.

To investigate the stability of the insulating state, see Eq. (109), we have to study the limit of $m=m^{*}(n), n \rightarrow \infty$, where $m^{*}(n)$ determines the direction to maximize $\ln W^{(n, m)}$ for fixed $n$.

As before, we will restrict ourselves by the condition

$$
I \simeq \lambda \ll \lambda M .
$$


Simple counting of combinatorial factors in Eq. (136) shows that under condition (137) maximum is achieved for the maximal possible number of the electron-hole pairs per a localization cell, $N_{i} \simeq T / \delta_{\zeta}$. It translates into estimate

$$
m^{*}(n) \simeq \frac{n \delta_{\zeta}}{T}
$$

Substituting Eq. (138) into Eq. (136), we find $\mathcal{C}\left[n, m^{*}(n)\right] \lesssim(2 d)^{m^{*}(n)}$ and obtain from Eq. (135):

$$
\ln W^{\left(n \gg 1, m^{*}(n)\right)}=-\left(s \Gamma^{(0,0)}\right)^{\gamma(T) / 2}\left(\frac{6 \mathrm{e} \lambda M T}{\delta_{\zeta}} I^{c_{1} \delta_{\zeta} / T} \ln \frac{\Delta}{\lambda \delta_{\zeta}}\right)^{n}
$$

where $c_{1}(d)$ is the number of the order of unity which we were not able to calculate, and all the other entries are the same as in Eq. (130a).

Applying stability criterion (109), we arrive to the main conclusion of this section - insulating state is stable only for $T<T_{c}$, where the critical temperature is given by

$$
\frac{6 \mathrm{e} \lambda M T_{c}}{\delta_{\zeta}} \ln \frac{\Delta}{\lambda \delta_{\zeta}}=\exp \left[\frac{c_{1} \delta_{\zeta}|\ln I|}{T_{c}}\right] .
$$

As $I$ is not exponentially small, $I \gg \mathrm{e}^{-1 / \lambda M}$, Eq. (140) may be expanded as

$$
T_{c}=T_{*}[1+\mathcal{O}(\lambda M|\ln I|)]
$$

where $T_{*}$ is given by Eq. (131).

At temperatures larger than $T_{c}$ metallic phase is formed. Together with the material of Sec. 5 proving the stability of the metallic phase at $T>T_{i n} \gg T_{c}$, see Eq. (80), this completes the proof of the existence of the metal insulator transition.

Kinetics of the system near the transition itself is a complicated problem which we hope to address in a separate publication. However, some conclusions can be drawn already from Eqs. (135), (136), and the estimate (138). Indeed, we concluded that the best paths are those that maximize the number of electron-hole pairs in a given localization cell before hopping to a neighbouring one. It means that the self-intersections of the random path in the Eq. (136) are forbidden and the spatial part of the problem becomes equivalent to the statistics of the self-avoiding random walk.

The latter observation enables us to conjecture the critical behavior of the spatial localization length $\zeta$ at $T \rightarrow T_{c}-0$. The latter length is defined from Eq. (136), as

$$
\zeta=\left|\sum_{i} \boldsymbol{a}_{i}\right|_{t y p}
$$


where the configurations giving the largest statistical weight in Eq. (136) are meant by typical. The correlation length in the Fock space (typical distance between resonances) diverges in the vicinity of the transition as [22]

$$
n_{t y p} \simeq \frac{T_{c}}{\left|T_{c}-T\right|}
$$

hence, the number of segments $m \simeq \delta_{\zeta} /\left|T_{c}-T\right|^{-1}$. We thus conclude

$$
\zeta(T) \simeq \zeta_{l o c}\left(\frac{\delta_{\zeta}}{T_{c}-T}\right)^{\nu_{d}}
$$

where $\nu_{d}$ is the correlation length index for the self-avoiding random walk in $d$ dimensions, $\nu_{1}=1, \nu_{2}=3 / 4, \nu_{3}=0.59 \ldots, \nu_{d>4}=1 / 2$, see Ref. [30].

\section{$7 \quad$ Validity of ImSCBA scheme}

This section is devoted to the analysis of the contributions of the processes not taken into account in our ImSCBA calculational scheme, see Figs. 4,5, and Eq. (68). The latter approximation corresponds to neglecting the level shifts due to the electron-electron interaction. The former will be shown to correspond to the renormalization of the electron-electron interaction by intermediate virtual processes, and to certain interference effects. We will consider the effects of those contributions separately in the following subsections.

We note here, however, that each remaining diagram, denoted by $\mathcal{U}$, is a random quantity. Therefore, we will have to analyse the statistical distribution of the remaining diagrams and check that their distribution functions have the scale parametrically smaller than the scale of $\Gamma_{l}(\epsilon)$ calculated in the previous section. The signs of the majority of the diagrams are random, so it is more convenient to use the characteristic function, see, e.g., Eq. (108), in a Fourier transform form

$$
W^{\mathcal{U}}(q)=\langle\exp (i q \mathcal{U})\rangle,
$$

where the averaging procedure is defined in Eq. (73). To calculate function (144), all the machinery of Sec. 6.3 is applicable after the replacement $s \rightarrow-i q$, and $\mathcal{U}$ on the Fig. 12 should be understood as an analytic expression for the real or imaginary part of the corresponding diagram.

We will present in detail the analysis for the insulating phase in Secs. $7.1-$ 7.3; the corresponding consideration for the developed metallic phase is simple and is summarized in Sec. 7.4. 


\subsection{Effect of the interaction renormalization.}

To understand the origin of the interaction renormalization, let us consider the contributions c5,c6 from Fig. 4, see also Fig. 16. These contributions are the only third-order terms that may lead to the finite decay rate - all others are either insignificant corrections to the Hartree-Fock potential $(\mathrm{c} 2, \mathrm{c} 3)$, or $(\mathrm{c} 1, \mathrm{c} 4)$ the first order Hartree-Fock shift in the second order diagram $(\mathrm{b} 1, \mathrm{~b} 3)$. Direct calculation of the diagram $(\mathrm{c} 5, \mathrm{c} 6)$ yields

$$
\begin{aligned}
& \operatorname{Im} \Sigma_{l}^{A(\operatorname{Fig} 4 c 5)}(\epsilon)=\frac{1}{2} \sum_{l_{1}, \ldots, l_{5}} \int \mathrm{d} \epsilon_{1} \ldots \mathrm{d} \epsilon_{5} A_{l_{1}}\left(\epsilon_{1}\right) \ldots A_{l_{5}}\left(\epsilon_{5}\right) \\
& \times \quad\left[V_{l l_{3}}^{l_{1} l_{2}} V_{l_{2} l_{5}}^{l_{3} l_{4}} V_{l_{4} l_{1}}^{l_{5} l} \pi \delta\left(\epsilon-\epsilon_{1}-\epsilon_{2}+\epsilon_{3}\right) F_{l_{1}, l_{2} ; l_{3}}^{\Rightarrow}\left(\epsilon_{1}, \epsilon_{2} ; \epsilon_{3}\right) P \frac{n_{l_{4}}\left(\epsilon_{4}\right)-n_{l_{5}}\left(\epsilon_{5}\right)}{\epsilon_{2}-\epsilon_{3}-\epsilon_{4}+\epsilon_{5}}\right. \\
& \quad+(2 \leftrightarrow 4 ; 3 \leftrightarrow 5)] ; \\
& \operatorname{Im} \Sigma_{l}^{A(\mathrm{Fig} 4 c 6)}(\epsilon)=\frac{1}{8} \sum_{l_{1}, \ldots, l_{5}} \int \mathrm{d} \epsilon_{1} \ldots \mathrm{d} \epsilon_{5} A_{l_{1}}\left(\epsilon_{1}\right) \ldots A_{l_{5}}\left(\epsilon_{5}\right) \\
& \quad \times\left[V_{l l_{3}}^{l_{1} l_{2}} V_{l_{1} l_{2}}^{l_{4} l_{5}} V_{l_{4} l_{5}}^{l_{3} l} \pi \delta\left(\epsilon-\epsilon_{1}-\epsilon_{2}+\epsilon_{3}\right) F_{l_{1}, l_{2} ; l_{3}}^{\Rightarrow}\left(\epsilon_{1}, \epsilon_{2} ; \epsilon_{3}\right) P \frac{n_{l_{4}}\left(\epsilon_{4}\right)+n_{l_{5}}\left(\epsilon_{5}\right)}{\epsilon_{1}+\epsilon_{2}-\epsilon_{4}-\epsilon_{5}}\right. \\
& \quad+(1 \leftrightarrow 4 ; 2 \leftrightarrow 5)],
\end{aligned}
$$

where the notation is the same in in Eqs. (69), and $P$ denotes the principal value. It is important to notice that using ImSCBA Green functions in this expression rather than the bare Green functions is not an overstepping of the accuracy of the calculation.

Expression (145) illustrates the well-known principle of constructing higher order contributions from the lower ones. Namely, to obtain the imaginary part of any contribution to the self-energy one can cut the diagram in all possible ways [cuts are shown by vertical dotted lines on Fig. 16]. The cross-section produces a $\delta$-function for the energies of the particles crossing the cut. The two parts of the diagrams on each side of the cut correspond to transition amplitudes. Being real, they can be incorporated into the redefinition of the constants of the initial Hamiltonian (for the clean Fermi liquid it was first realized by Eliashberg [31]).

For example, expression (145) can be obtained from $\Gamma_{l}^{(i n)}(\epsilon)$ of Eq. (69a) by the replacement of the bare interaction matrix element $V_{l_{1} l_{2}}^{j_{1} j_{2}}$ with the potential renormalized by excitation of virtual particle-hole pairs, $\delta V_{e h}$, or particle- 
particle pairs, $\delta V_{e e}$, see Fig. $16 \mathrm{~b}$ :

$$
\begin{aligned}
& V_{l_{1}, l_{2}}^{l_{3} l_{4}} \rightarrow V_{l_{1}, l_{2}}^{l_{3} l_{4}}+\left[\delta V_{e h}\right]_{l_{1}, l_{2}}^{l_{3} l_{4}}-\left[\delta V_{e h}\right]_{l_{2}, l_{1}}^{l_{3} l_{4}}+\left[\delta V_{e e}\right]_{l_{1}, l_{2}}^{l_{3} l_{4}} \\
& {\left[\delta V_{e h}\right]_{l_{1}, l_{2}}^{l_{3} l_{4}}=\frac{1}{2} \sum_{l_{5}, l_{6}} \int \mathrm{d} \epsilon_{5} \mathrm{~d} \epsilon_{6} A_{l_{5}}\left(\epsilon_{5}\right) A_{l_{6}}\left(\epsilon_{6}\right) V_{l_{1} l_{5}}^{l_{3} l_{6}} V_{l_{6} l_{2}}^{l_{5} l_{4}} P \frac{n_{l_{6}}\left(\epsilon_{6}\right)-n_{l_{5}}\left(\epsilon_{5}\right)}{\epsilon_{2}-\epsilon_{3}-\epsilon_{6}+\epsilon_{5}} ;} \\
& {\left[\delta V_{e e}\right]_{l_{1}, l_{2}}^{l_{3} l_{4}}=\frac{1}{4} \sum_{l_{5}, l_{6}} \int \mathrm{d} \epsilon_{5} \mathrm{~d} \epsilon_{6} A_{l_{5}}\left(\epsilon_{5}\right) A_{l_{6}}\left(\epsilon_{6}\right) V_{l_{1} l_{2}}^{l_{5} l_{6}} V_{l_{5} l_{6}}^{l_{3} l_{4}} P \frac{n_{l_{6}}\left(\epsilon_{6}\right)+n_{l_{5}}\left(\epsilon_{5}\right)}{\epsilon_{1}+\epsilon_{2}-\epsilon_{6}-\epsilon_{5}} ;}
\end{aligned}
$$

where we are using the short-hand notation $l_{i} \equiv\left(l_{i}, \epsilon_{i}\right)$ in the first line of the expression.

For the insulating regime we use the leading term in Eq. (103), $A_{l}(\epsilon) \approx$ $\delta\left(\epsilon-\xi_{l}\right)$, and calculate the characteristic functions $W^{\delta V_{e e}}(q)$ and $W^{\delta V_{e h}}(q)$ as shown on Fig. 16c. Assuming $\left|\epsilon_{2}-\epsilon_{3}\right|,\left|\epsilon_{2}+\epsilon_{1}\right| \gtrsim \delta_{\zeta}, T \gtrsim M \delta_{\zeta}$, we find

$$
\begin{aligned}
& \ln W^{\delta V_{e h}}(q)=\left\{\begin{array}{l}
-2 \pi|q| \lambda^{2} T ;\left|\epsilon_{2}-\epsilon_{3}\right| \lesssim M \delta_{\zeta} ; \\
-\frac{\pi|q| \lambda^{2} M \delta_{\zeta}}{2} ;\left|\epsilon_{2}-\epsilon_{3}\right| \gtrsim M \delta_{\zeta} ;
\end{array}\right. \\
& \ln W^{\delta V_{e e}}(q)=-\frac{\pi|q| \lambda^{2} M \delta_{\zeta}}{2} .
\end{aligned}
$$

Equations (147) describe random quantities with the characteristic scale of the distribution $|\delta V|_{\text {typ }}$ given by the coefficient multiplying $|q|$. This width should be compared with the bare value of the interaction constant $\lambda \delta_{\zeta}$. For $T \leq T_{*}$, [see Eq. (131)], we find

$$
\frac{|\delta V|_{t y p}}{\lambda \delta} \simeq \frac{1}{M}\left(\frac{T}{T^{*}}\right)
$$

i.e., the typical value of the correction to the interaction constant is parametrically smaller than the bare value. On the other hand, the distribution of the $\delta V$ has the same algebraic decay at large values as the distributions of the ImSCBA quantities $\Gamma$, see, e.g., Eq. (129). It means that substitution of the renormalized constant $\delta V$ in, say, second order perturbation theory formula (114) will produce the distribution function of the form as the fourth order perturbation theory result (125), but with the coefficient smaller at least by the factor of $M$. The same is true for any order, and therefore, the interaction renormalization (147) can produce only perturbative in $1 / M$ corrections to the value of the transition temperature $T_{c}$.

It is not difficult to see that certain cross-sections of higher-order diagrams may be ascribed to the higher-order corrections to the interaction vertex, see 
a)

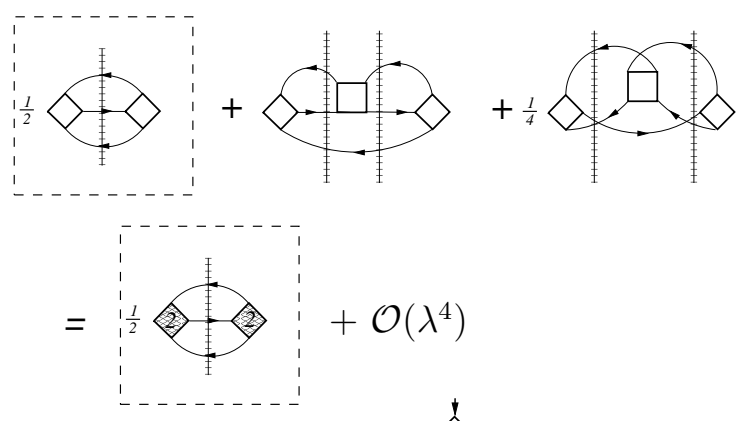

b)

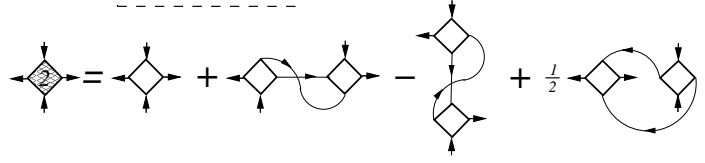

c)

$$
\ln W^{\delta V_{e h}}=\underbrace{\delta V_{e e}}_{\frac{1}{2}}
$$

Fig. 16. Reduction of the third order diagrams (a) to the renormalization of the interaction constant in the SCBA scheme (b). Calculation of the characteristic function (c) using notation of Fig. 12.

Fig. 17. However, not all of the cross-sections can be taken into account in a such a fashion, see, e.g., cross-section (c2) of Fig. 5, or Fig. 18a. Remaining terms describe the effects of the particle permutations in the final state which will be discussed in the following subsection, see Fig. 19, and generation of the interaction vertices involving a larger number of the particles, see Fig. 18c.

Statistical analysis of the higher-order corrections to the vertices is performed in the same fashion and produce distributions similar to that of Eqs. (147) with the smallness (148) in higher and higher powers. We thus conclude that the vertex renormalization does not lead to any dramatic effects; it only produces perturbative corrections to the transition temperature $T_{c}$ calculated within ImSCBA scheme.

\subsection{Effect of the particle permutations in the final state.}

Cross-sections of the fourth-order diagrams, not included in Fig. 17, are shown in Fig. 19 (5.1a-5.2d) together with the two ImSCBA cross-sections (5.1 and 5.2). One can notice that all of these cross-sections contain the sum over the same final 5-particle states (the same orbital indices of the particles and holes 


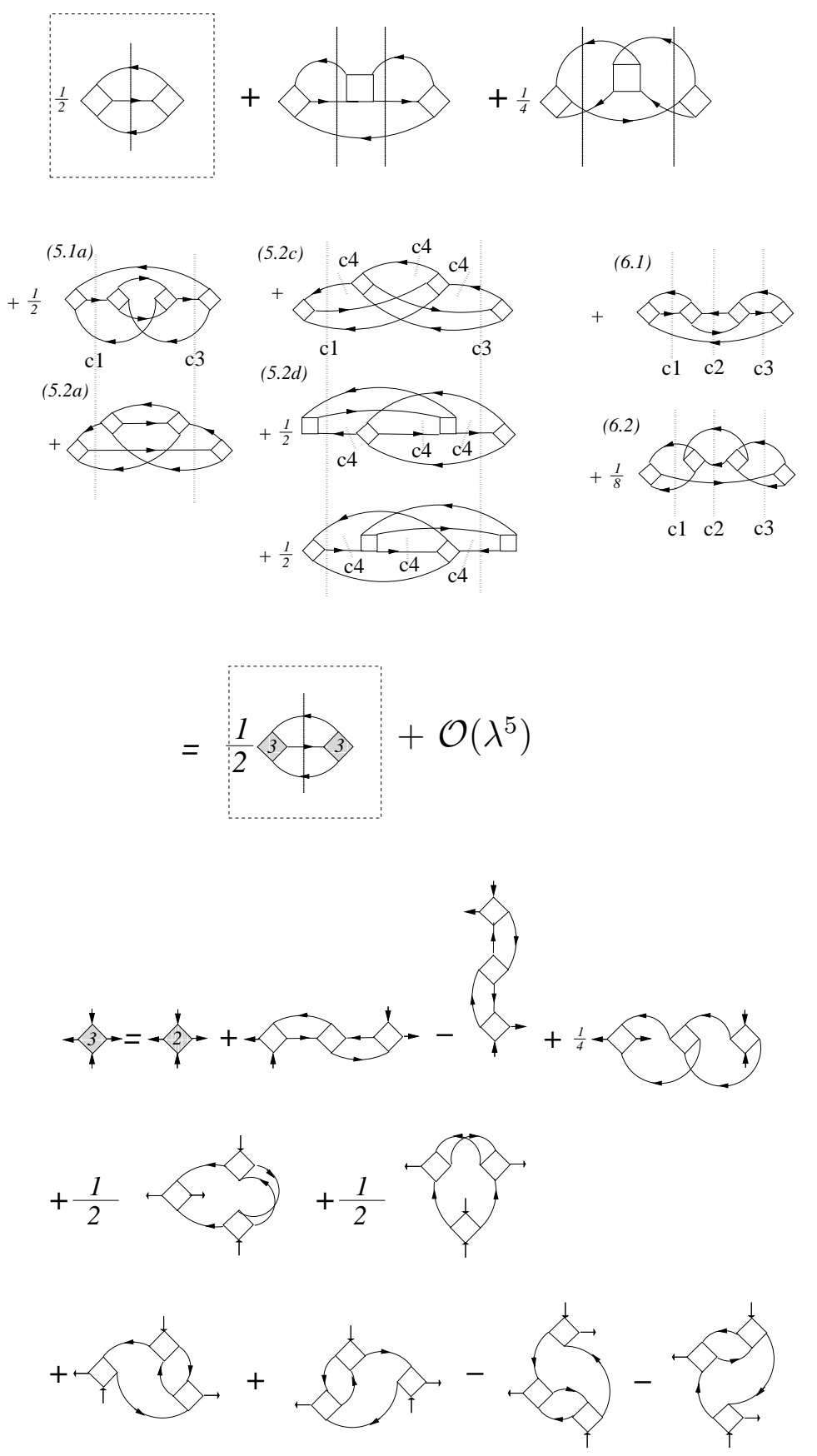

Fig. 17. Reduction of the certain cross-section of fourth order diagrams, Fig. 5, to the renormalization of the interaction constant in the SCBA scheme.

crossing the cut). Moreover, the analytic expressions corresponding to the two parts of a cut ImSCBA diagram for a given final state (transition matrix elements) are equal. For non-ImSCBA diagrams the two resulting expressions are different, but they always can be reduced to the transition matrix elements for the diagrams 5.1 and 5.2 by a permutation of the orbital indices of the particles in the final state. This observation enables us to identify the non-ImSCBA cross-sections $5.1 \mathrm{a}-5.2 \mathrm{~d}$ as interference terms in the transition 

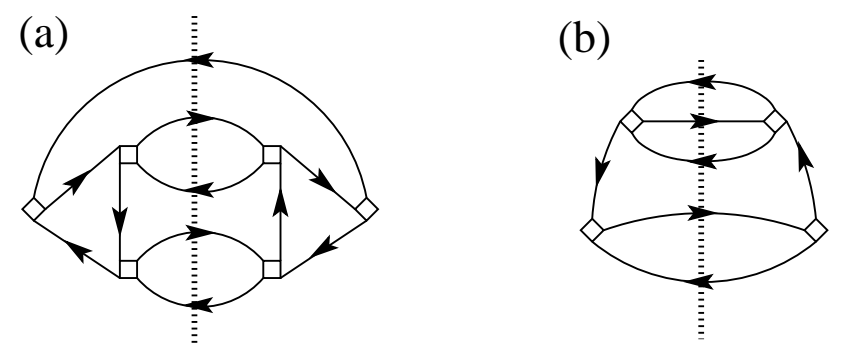

(c)

$$
\overbrace{-i q} \simeq-|q| \lambda^{3} T \simeq-|q| \lambda^{2} \delta_{\zeta}\left(\frac{T}{M T_{*}}\right)
$$

(d)

$$
\sum_{-i q}=-\pi|q| \lambda^{2} \delta_{\zeta}
$$

Fig. 18. a) Reduction of a crosssection of the 6 -th order diagrams to the three particle interaction. b) SCBA diagram generating the same state in the crosssection. c-d) Comparing of the distribution functions of the non-equivalent blocks in the SCBA diagram (d) and in the 3-particle interaction diagram (c).

probability, while ImSCBA corresponds to the replacement of the square of the sum in Fig. 19 by the sum of squares of individual terms.

This consideration for the fourth-order self-energy illustrates the general rule of construction of all lowest-order diagrams containing a cross-section of $N_{e}$ electrons and $N_{h}$ holes. Let us assume for a moment that electrons and holes are distinguishable particles and introduce the quantum-mechanical amplitude $\mathcal{A}_{\ell}\left(\left\{l_{i}\right\}_{i=1}^{N_{e}} ;\left(\left\{m_{i}\right\}_{i=1}^{N_{h}}\right)\right.$ of the transition to the given final state for a given path $\ell$ (represented by a half-diagram with a definite assignment of orbital indices to the free electron and hole lines). The decay rate can then be represented as

$$
\begin{aligned}
\Gamma_{l} & =\sum_{\left\{l_{i}\right\},\left\{m_{i}\right\}} \gamma_{l}\left(\left\{l_{i}\right\},\left\{m_{i}\right\}\right) \delta\left(\epsilon-\sum_{i=1}^{N_{e}} \xi_{l_{i}}+\sum_{i=1}^{N_{h}} \xi_{m_{i}}\right) \\
\gamma_{l} & =\frac{1}{N_{e} ! N_{h} !}\left|\sum_{\ell} \sum_{P_{l}, P_{m}}(-1)^{P_{l}+P_{m}} \mathcal{A}_{\ell}\left(P_{l}\left\{l_{i}\right\}, P_{m}\left\{m_{i}\right\}\right)\right|^{2} .
\end{aligned}
$$

The factorial prefactor takes into account the fact that different permutations of the indices $\left\{l_{i}\right\},\left\{m_{i}\right\}$ correspond to the same final state, the sum over the permutations represents the usual antisymmetrization of the quantum- 


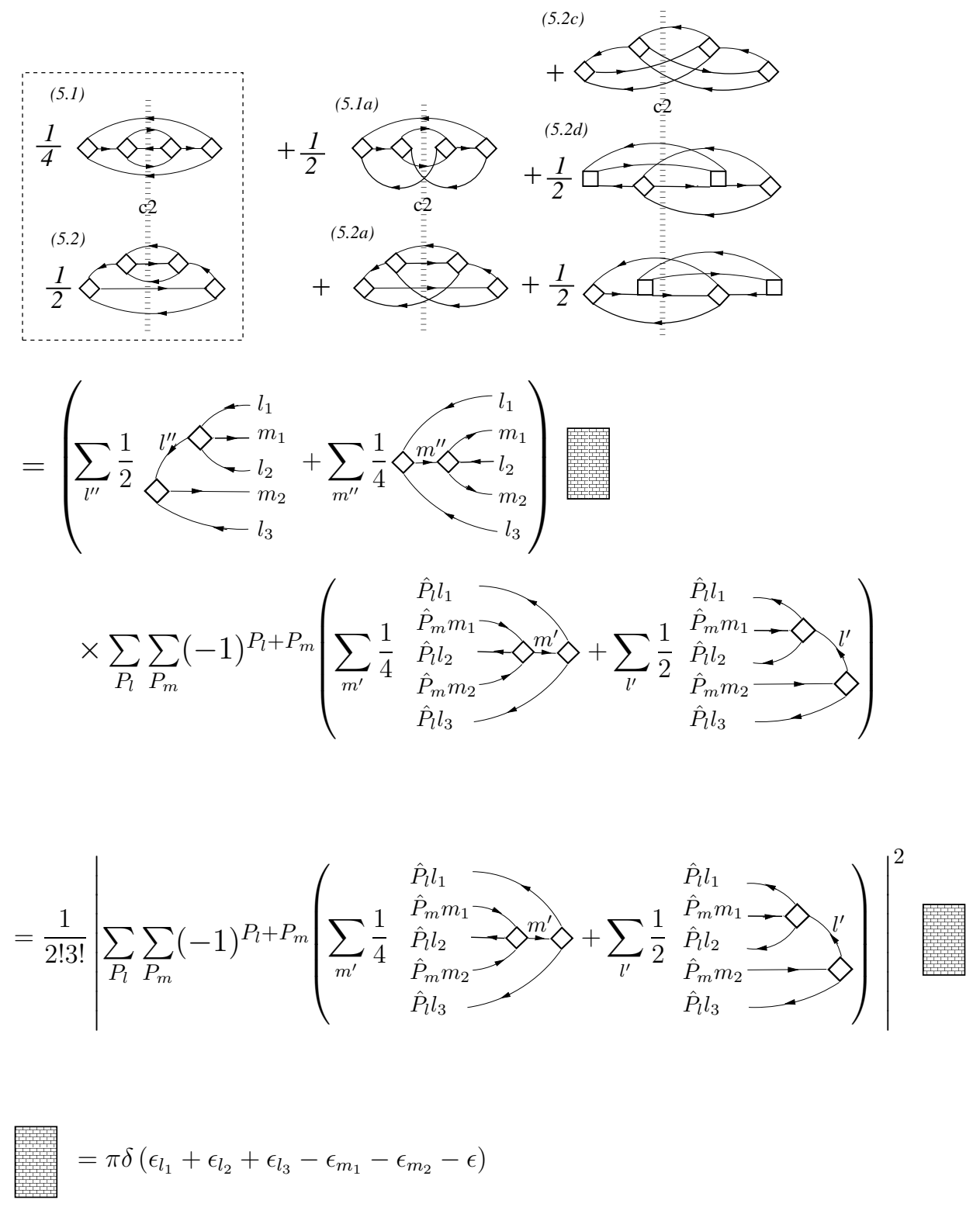

Fig. 19. Cross-sections of the fourth-order diagrams for self-energy, not included in Fig. 17. The orbital indices of the particles crossing the cut $\left(l_{1}, l_{2}, l_{3}, m_{1}, m_{2}\right)$ are summed over. For fixed values of these indices each of the two parts of the diagram with the free ends removed corresponds to the partial transition matrix element. Summation of the latter over all permutations of the orbital indices of identical particles in the final state gives the total transition matrix element for a given final state. The permutations $\hat{P}_{l}$ and $\hat{P}_{m}$ are independent; they act on electron $\left(l_{1}, l_{2}, l_{3}\right)$ and hole $\left(m_{1}, m_{2}\right)$ indices, respectively. The decay rate is then given by the square of the total matrix element, summed over all final states. Since different permutations of electron and hole indices give the same final state, the factor $1 /(3 ! 2 !)$ in front of the sum is necessary. Diagrams (2) and (4) of Fig. 7c are also generated for $l^{\prime \prime} \neq l^{\prime}$ or $m^{\prime \prime} \neq m^{\prime}$. 


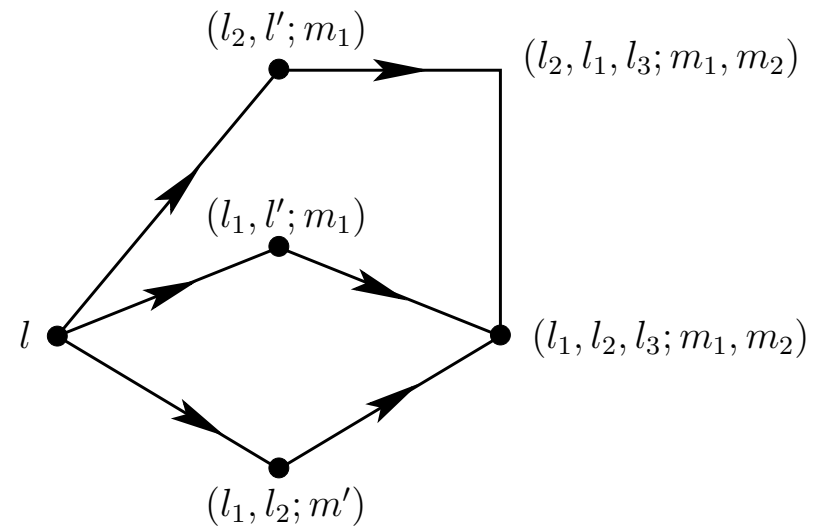

Fig. 20. Different paths for the transition from a one-particle state $l$ to a five-particle state $\left(l_{1}, l_{2}, l_{3} ; m_{1}, m_{2}\right)$, corresponding to different intermediate three-particle states. The state $\left(l_{2}, l_{1}, l_{3} ; m_{1}, m_{2}\right)$ is identical to $\left(l_{1}, l_{2}, l_{3} ; m_{1}, m_{2}\right)$.

mechanical amplitude ${ }^{16}$.

Similarly to the consideration of the quantum interference effects for a single particle in a disorderd potential, the resulting double sum in Eq. (149) can be separated into diagonal and off-diagonal part

$$
\Gamma_{l} \propto \sum_{\ell}\left|\mathcal{A}_{\ell}\left(\left\{l_{i}\right\},\left\{m_{i}\right\}\right)\right|^{2}+\text { (off-diag.) }
$$

The first term in Eq. (150) is nothing but the ImSCBA series corresponding to the summation of the probablities of the paths. Random-sign last term is the quantum interference contribution to the particle lifetime.

Even though the number of the interference terms is much larger than the number of diagonal terms one can still argue that they do not affect the value of the transition temperature.

Indeed, if the number of the relevant terms were large, one would be able to apply the central limit theorem for the quantum-mechanical amplitudes rather than for the probabilities. It would result in a distribution function of the same scale as the one obtained by the diagonal approximation but of a different shape (e.g., for the large number of statiscally independent amplitudes Porter-Thomas distribution would replace the Gaussian one). However, the characteristic functions obtained in Sec. 6.4 are non-Gaussian (as they are not analytic at $s \rightarrow 0$ ) and have long algebraic tails at large values of $\Gamma$. This indicates that the result is contributed by the largest term in the sum. Therefore, the interference contribution affects the distribution function in the range of the most probable values but not the tail of the distribution. As the

${ }^{16}$ It is easy to see that the diagrams (2) and (4) of Fig. 7c are also included in Eq. (149) 
transition temperature is controlled by the tail, the interference term is not important for the position of this temperature. ${ }^{17}$

To quantify the qualitative consideration above we evaluated the characteristic function for the quantity on the right-hand side of Eq. (149), calculated in the $n$th order of the perturbation theory $(n \gg 1)$,

$$
\begin{aligned}
& \ln \left\langle\exp \left(-s \delta_{\zeta}^{-2} \sum_{\left\{l_{i}\right\},\left\{m_{i}\right\}} \gamma_{l}\left(\left\{l_{i}\right\},\left\{m_{i}\right\}\right)\right)\right\rangle=-\sqrt{s}\left(\frac{T}{T_{*}}\right)^{n}\left(1-\sqrt{s \alpha_{i n t}}+\ldots\right) \\
& \alpha_{\text {int }} \simeq\left[\lambda^{n} n !\right]^{2} .
\end{aligned}
$$

Derivation of Eq. (151) is relegated to Appendix D, and we neglected $\ln \lambda$ factors in the expression for $\alpha_{i n t}$.

Leading at $s \rightarrow 0$ term is none but the the diagonal contribution calculated in SCBA approximation. The subleading term is the interference term, and the parameter $\alpha_{i n t}$ has a meaning of the relative contribution of the off-diagonal terms, affecting the distribution at small values of $\gamma$. Values of $\alpha_{\text {int }}$ contain the $n$ ! factor, so it apparently becomes large no matter how small the interaction constant $\lambda$ is. However, as we discussed in the derivation of Eq. (132), the order of the perturbation theory involving only one localization cell is limited from above, $n<n^{*} \simeq T / \delta_{\zeta}$. As the result for $I=0, n=n^{*}$ we estimate

$$
\alpha_{i n t} \simeq\left[\frac{\lambda T}{\delta_{\zeta}}\right]^{2 n^{*}} \simeq M^{-2 n^{*}} \ll 1,
$$

which means that the interference processes do not affect the most important part of the distribution functions even in the largest possible order of the perturbation theory without hopping between localization cells.

One could think that the inclusion of the hopping would allow the growth of the $\alpha_{\text {int }}$ beyond the estimate (152) at $n>n^{*}$. However, it is not the case. Inclusion of tunneling into neighboring localization cells will lead to further suppression of the interference effects. The presence of tunneling vertices on the diagram makes it impossible to interchange particles residing in different localization cells, compare Figs. 19 and 21. It is possible to check that keeping the combinatorial $n$ ! in the perturbation theory involving $n$ particles in the final states spread over $m \ll n$ localization cells would require the tunneling of

\footnotetext{
${ }^{17}$ Moreover, experience gained in the study of the critical behavior of the Anderson transition on the Cayley tree [22] suggests that the transition itself is associated with the reconstruction of the tail of the distribution. Therefore, we do not see any reason to believe the the interference terms can affect the critical behavior in our problem either.
} 

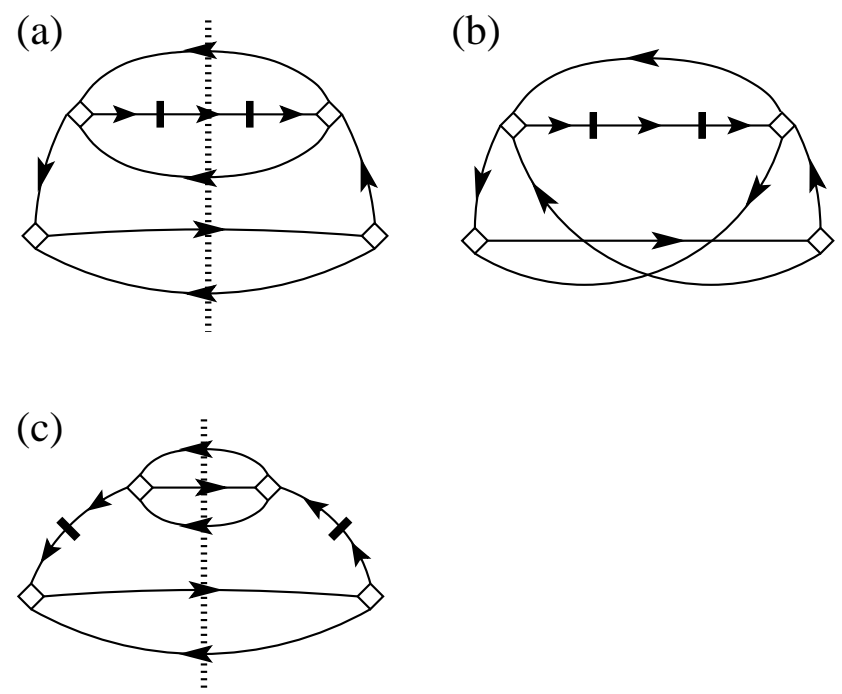

Fig. 21. Suppression of the exchange processes shown on Fig. 19 due to the inclusion of the tunneling vertices. Diagram a) has only one exchange counterpart b) whereas the diagram c) does not allow permutations at all [ $c f$. diagram (5.2) which produces three exchange counterparts].

all $n$ particles by the distance of the order of $m$. Each tunneling event brings additional smallness $I$, and thus we estimate

$$
\alpha_{i n t} \simeq\left[\lambda^{n} n ! I^{m n}\right]^{2} \lesssim\left[n \lambda \exp \left(-\frac{n|\ln I| \delta_{\zeta}}{T}\right)\right]^{2 n}
$$

where we used $m \simeq m^{*}$ from Eq. (138). Thus, no accumulation of the factorial terms is possible, even in the vicinity of the metal-insulator transition, and the SCBA calculational scheme is valid not only on qualitative but also on a quantitative level.

\subsection{Effect of the single-particle spectrum renormalization.}

We now turn to the study of the effects on the interaction and tunneling which may be viewed as a change in the properties of single-particle excitations. This change includes the level shifts $\delta \xi_{l}$ and the possibility of mixing with other orbitals (which is present even without interaction due to the hopping $I$ ). The level shifts are encoded in the statistics of the real parts of the diagonal part of the self-energy $\operatorname{Re} \Sigma_{l}^{R}$ which were neglected in ImSCBA approximation, whereas the bubbles non-diagonal with respect to the initial and final states, see, e.g., diagram (c3) of Fig. 7, are responsible for the mixing. 
The lowest order diagram is the Hartree-Fock potential of Fig. 4a

$$
U_{l_{1} l_{2}}^{H F}=-\frac{1}{2} \sum_{l_{3}}\left[n_{l_{3}}-\operatorname{sgn} \xi_{l_{3}}\right] V_{l_{1} l_{3}}^{l_{3} l_{2}}
$$

where we subtracted the value of the potential at $T=0$. The latter term is assumed to be incorporated into the one-particle Hamiltonian. The characteristic function (144) is found using the averaging procedure (73) and Eq. (48g) to be Gaussian

$$
\ln W^{U^{H F}}=-q^{2} \lambda^{2} \delta_{\zeta} T \ln 2 \sim-q^{2} \delta_{\zeta}^{2}\left(\frac{\lambda}{M}\right)\left(\frac{T}{T_{*}}\right)
$$

This means that the variation of the Hartree-Fock potential with the distribution function is much smaller than the level spacing $\delta_{\zeta}$ even for $T$ of the order of transition temperature $T_{c}$.

The Hartree-Fock diagram as well as other diagrams, corresponding to singleparticle level shifts and mixing, may be included as self-energy insertions into single-particle Green's functions in all diagrams of the previous subsections. As a result, the Green's functions become non-diagonal both in the orbital indices $l$, and the spatial index $\boldsymbol{\rho}$, if tunneling is involved. Such insertions always introduce an additional smallness, as discussed in Sec. 6.5 for the case of tunneling in the ImSCBA. However, while inclusion of tunneling is necessary to go beyond the finite state space of a single localization volume, insertions non-diagonal only in the orbital index do not change the number of the final states and thus can be ignored. Insertion of tunneling into the renormalized interaction vertex, see Fig. 22a, produces interaction, nonlocal in space. However, the corresponding correction to $\Gamma_{l}$ is "small" compared to that of the ImSCBA diagram with Fig. 22b with the same final states, see Fig. 22c,d. Thus we conclude the variations of the Hartree-Fock potential do not generate any corrections to the transition temperature or the statistics of $\Gamma_{l}$ calculated in ImSCBA.

However, there is an important feature in the statistics of the level shifts $\xi_{l}$ which changes the numerical factor in Eq. (140) and changes the power-law decay in the tail of the distribution of $\Gamma_{l}$. To see this, let us consider now the second-order contribution shown on Fig. 4b.2. Real part of this self-energy $\delta \xi_{l}(\epsilon)$ is included in SCBA but neglected in ImSCBA. To investigate its effect, we apply the Kramers-Kronig relation to $\Gamma_{l}^{(i n)}(\epsilon)$ from Eq. (69a):

$$
\delta \xi_{l}(\epsilon)=\lambda^{2} \delta_{\zeta}^{2} \sum_{l_{1}, l_{2}, l_{3}} Y_{l_{1}, l_{2}}^{l_{3}, l} \int \mathrm{d} \epsilon_{1} \mathrm{~d} \epsilon_{2} \mathrm{~d} \epsilon_{3} F_{l_{1}, l_{2} ; l_{3}}^{\Rightarrow}\left(\epsilon_{1}, \epsilon_{2} ; \epsilon_{3}\right) P \frac{A_{l_{1}}\left(\epsilon_{1}\right) A_{l_{2}}\left(\epsilon_{2}\right) A_{l_{3}}\left(\epsilon_{3}\right)}{\epsilon-\epsilon_{1}-\epsilon_{2}+\epsilon_{3}}
$$

where all the notation is introduced in Eq. (69a). Substituting $A_{l_{i}}\left(\epsilon_{i}\right)=\delta\left(\epsilon_{i}-\right.$ 
(a)

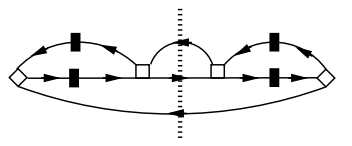

(b)

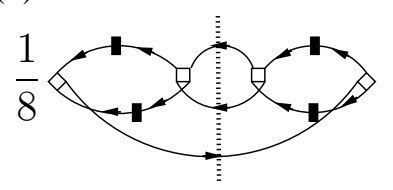

(c)
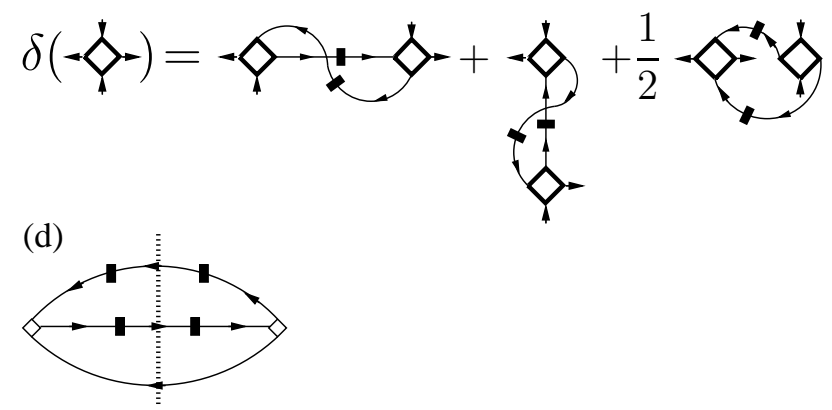

(e)

$\gamma_{-i q} \simeq|q| \lambda^{2} I^{2} T \ln ^{2}\left(|q| \delta_{\zeta} \lambda^{2} I^{2}\right)$

(f)

$\sum_{-i q} \simeq|q| \delta_{\zeta} \lambda I^{2} \ln \left(|q| \delta_{\zeta} \lambda I^{2}\right)$

Fig. 22. Reduction of the non-SCBA diagrams (a-b) to the interaction vertex non-local in space (c). Characteristic function of the non-local interaction (c) in comparison with the corresponding block (f) of the SCBA diagram (d).

$\left.\xi_{l_{i}}\right)$, we obtain

$$
\delta \xi_{l}(\epsilon)=\lambda^{2} \delta_{\zeta}^{2} \sum_{l_{1}, l_{2}, l_{3}} \frac{Y_{l_{1}, l_{2}}^{l_{3}, l} F_{l_{1}, l_{2} ; l_{3}}}{\epsilon-\xi_{l_{1}}-\xi_{l_{2}}+\xi_{l_{3}}}
$$

whose characteristic function is

$$
\ln W^{\delta \xi}=-2 \pi|q| \lambda^{2} M T \simeq-|q| \lambda \delta_{\zeta}\left(T / T_{*}\right)
$$

Therefore, the typical value of $\delta \xi \sim \lambda \delta_{\zeta} \ll \delta_{\zeta}$. One thus might think that the main effect of this contribution is a weak random shift of the level position, so it can be disregarded as well.

Nevertheless, despite its smallness, $\delta \xi_{l}(\epsilon)$ introduces a new qualitative effect: repulsion between many-body levels. When $\delta \xi_{l}(\epsilon)$ is taken into account, one has to find the position of the shifted single-particle level from the equation

$$
\epsilon-\xi_{l}-\delta \xi_{l}(\epsilon)=0
$$

This equation describes repulsion between the single-particle excitation $l$ and 
three-particle excitations $\left(l_{1}, l_{2} ; l_{3}\right)$ : solutions of Eq. (159) cannot approach each other by a distance smaller than $\lambda \delta_{\zeta}$.

In the considerations of Sec. 6 we assumed the energies of one-particle states to be independent of the energies of three-particle states into which these oneparticle states decayed. As a result, resonant denominators [see, e.g., Eq. (123)] could become small independently of each other. The level repulsion suppresses such effect, so that Eqs. (130a), (132), and (135) overestimate the strength of the tail of the distribution.

It is the same effect that was first discussed by Anderson [1] and analyzed rigorously by Abou-Chacra et al. [17]. According to their results, this effect leads to a change in the numerical coefficient in the equation for the critical disorder strength: e $\rightarrow 2$. Simply adapting this prescription for our Eq. (140) we obtain the transition temperature modified by level repulsion

$$
\frac{12 \lambda M T_{c}}{\delta_{\zeta}} \ln \frac{1}{\lambda}=\exp \left[\frac{c_{1} \delta_{\zeta}|\ln I|}{T_{c}}\right]
$$

Now we will sketch the derivation of Eq. (160) for our problem. First, we have to identify the sequence of the diagrams which may give the level repulsion between the resonant multiparticle states. To do so, we include the level shift $\delta \xi_{l}(\epsilon)$ into the imaginary part of the fourth order SCBA diagram as shown on Fig. 23a.

Direct inspection of the diagrams Fig. 23a shows that for the levels $\xi_{l_{1}}, \ldots, \xi_{l_{6}}$ maximizing the skeleton diagram (a0) of Fig. 23, only diagrams (a1) and (a2) give rise to the simultaneous divergence of $\delta \xi$, whereas in the remaining diagrams (a3)-(a6) $\delta \xi$ fluctuates independently of $\Gamma_{l_{1}}$.

It means that the role of the level shift $\delta \xi$ in the diagrams (a3)-(a6), is, indeed, just the broadening of the distribution of $\xi_{l}$ by the value of $\lambda \delta_{\zeta}\left(T / T_{*}\right) \ll \delta_{\zeta}$, see Eq. (158). This broadening does not significantly change the distribution of the resonant denominators and can be disregarded together with the fluctuations of the Hartree-Fock potential. On the other hand, the level shifts on diagrams (a1), (a2) are large whenever $\Gamma_{l_{1}}$ is maximal and they describe the effect of level repulsion discussed before.

The maximally divergent series incorporating the correlations in $\delta \xi_{l}$ and $\Gamma_{l}$ is generated by the linearized self-consitent-Born approximation (LSCBA) shown on Fig. 23b, as it can be checked by explicit consideration of several iterations.

The analytic expression of the LSCBA self-energy is [cf. Eqs. (69a), (105a))]: 


$$
\begin{aligned}
& \delta \xi_{l}(\epsilon)= \sum_{l_{1}, \boldsymbol{a}} \frac{I^{2} \delta_{\zeta}^{2} \theta_{\Delta}\left[\epsilon-\xi_{l_{1}}(\boldsymbol{\rho}+\boldsymbol{a})\right]}{\epsilon-\xi_{l_{1}}(\boldsymbol{\rho}+\boldsymbol{a})-\delta \xi_{l_{1}}(\epsilon ; \boldsymbol{\rho}+\boldsymbol{a})} \\
&+ \lambda^{2} \delta_{\zeta}^{2} \sum_{l_{1}, l_{2}, l_{3}} Y_{l_{1}, l_{2}}^{l_{3}, l} F_{l_{1}, l_{2} ; l_{3}}^{\Rightarrow}\left[\frac{2 \theta_{\Delta}\left(\epsilon-\Xi_{l_{1} l_{2}}^{l_{3}}\right)}{\epsilon-\Xi_{l_{1} l_{2}}^{l_{3}}-\delta \xi_{l_{1}}\left(\epsilon-\Xi_{l_{2}}^{l_{3}}\right)}\right. \\
&\left.+\frac{\theta_{\Delta}\left(\epsilon-\Xi_{l_{1} l_{2}}^{l_{3}}\right)}{\epsilon-\Xi_{l_{1} l_{2}}^{l_{3}}+\delta \xi_{l_{3}}\left(\Xi_{l_{1} l_{2}}-\epsilon\right)}-\frac{2 \theta_{\Delta}\left(\epsilon-\Xi_{l_{1} l_{2}}^{l_{3}}\right)}{\epsilon-\Xi_{l_{1} l_{2}}^{l_{3}}}\right] ; \\
& \Gamma_{l}(\epsilon)=\Gamma_{l}^{(b a t h)}(\epsilon)+\sum_{l_{1}, \boldsymbol{a}} \frac{I^{2} \delta_{\zeta}^{2} \theta_{\Delta}\left[\epsilon-\xi_{l_{1}}(\boldsymbol{\rho}+\boldsymbol{a})\right] \Gamma_{l}(\epsilon, \boldsymbol{\rho}+\boldsymbol{a})}{\left[\epsilon-\xi_{l_{1}}(\boldsymbol{\rho}+\boldsymbol{a})-\delta \xi_{l_{1}}(\epsilon ; \boldsymbol{\rho}+\boldsymbol{a})\right]^{2}} \\
&+\sum_{l_{1}, l_{2}, l_{3}} \lambda^{2} \delta_{\zeta}^{2} Y_{l_{1}, l_{2}}^{l_{3}, l} F_{l_{1}, l_{2} ; l_{3}}^{\Rightarrow}\left\{\frac{2 \theta_{\Delta}\left(\epsilon-\Xi_{l_{1} l_{2}}^{l_{3}}\right) \Gamma_{l_{1}}\left(\epsilon-\Xi_{l_{2}}^{l_{3}}\right)}{\left[\epsilon-\Xi_{l_{1} l_{2}}^{l_{3}}-\delta \xi_{l_{1}}\left(\epsilon-\Xi_{l_{2}}^{l_{3}}\right)\right]^{2}}\right. \\
&\left.+\frac{\theta_{\Delta}\left(\epsilon-\Xi_{l_{1} l_{2}}^{l_{3}}\right) \Gamma_{l_{3}}\left(\Xi_{l_{1} l_{2}}-\epsilon\right)}{\left[\epsilon-\Xi_{l_{1} l_{2}}^{l_{3}}+\delta \xi_{l_{3}}\left(\Xi_{l_{1} l_{2}}-\epsilon\right)\right]^{2}}\right\}
\end{aligned}
$$

As in Eq. (69a), the coordinate $\boldsymbol{\rho}$ is assumed to be the same in all terms in the equations unless it is specified explicitly otherwise. The notation $\Xi_{l_{1} \ldots}^{m_{1} \ldots}$ was introduced in Eq. (113). The ultraviolet cutoff function $\theta_{\Delta}(x)$ is the same as in Eq. (104). It is important to emphasize that it depends on the unshifted energies of the levels. This is because the cutoff was introduced in the noninteracting Green function first; the self-energy appears in the denominator of the SCBA-dressed Green function as a result of the summation of a geometric series; such summation changes the denominator only, keeping the cutoff intact.

Now let us go through the steps of Sec. 6.4 and see how they are affected by the shifts in the denominators of Eqs. (161). First, we notice that Eq. (161b) is still given by the diagrammatic representation of Fig. 11 with the change in the rules of reading the double line:

$$
\stackrel{\epsilon}{\bar{l} \leftarrow}=\frac{\theta_{\Delta}\left(\epsilon-\xi_{l}\right)}{\left[\epsilon-\xi_{l}-\delta \xi_{l}(\epsilon)\right]^{2}} .
$$

The lowest order term of the cluster expansion, Eq. (114), see also Fig. 13, becomes

$$
\begin{aligned}
\Gamma^{(1,0)} & =2 \Gamma^{(0,0)} \sum_{l_{1}, l_{2}, l_{3}} \frac{\lambda^{2} \delta_{\zeta}^{2} Y_{l_{1}, l_{2}}^{l_{3}, l} F_{l_{1}, l_{2} ; l_{3}}^{\Rightarrow} \theta_{\Delta}\left(\epsilon-\Xi_{l_{1} l_{2}}^{l_{3}}\right)}{\left[\epsilon-\Xi_{l_{1} l_{2}}^{l_{3}}-\delta \xi_{l_{1}}\left(\epsilon-\Xi_{l_{2}}^{l_{3}}\right)\right]^{2}} \\
& +\Gamma^{(0,0)} \sum_{l_{1}, l_{2}, l_{3}} \frac{\lambda^{2} \delta_{\zeta}^{2} Y_{l_{1}, l_{2}}^{l_{3}, l_{l_{1}}} F_{l_{1}, l_{2} ; l_{3}}^{\Rightarrow} \theta_{\Delta}\left(\epsilon-\Xi_{l_{1} l_{2}}^{l_{3}}\right)}{\left[\epsilon-\Xi_{l_{1} l_{2}}^{l_{3}}+\delta \xi_{l_{3}}\left(\Xi_{l_{1} l_{2}}-\epsilon\right)\right]^{2}} .
\end{aligned}
$$


We see that the corresponding potential is no longer three-particle, but depends on all the coordinates via $\delta \xi$, which depend on the positions of all other levels. However, when performing the linked cluster expansion, in each term of the sum over triples $\left(l_{1}, l_{2}, l_{3}\right)$ we can shift an integration variable: $\xi_{l_{1}}+\delta \xi_{l_{1}} \rightarrow \xi_{l_{1}}$. After this shift Eq. (15) acquires the form

$$
\begin{aligned}
& \ln W_{3}^{(1,0)}=\frac{1}{2} \prod_{l=1}^{3}\left(\int \frac{\mathrm{d} \xi_{l}}{\delta_{\zeta}} \sum_{n_{l}= \pm 1} \frac{\exp \frac{n_{l} \xi_{l}}{2 T}}{2 \cosh \frac{\xi_{l}}{2 T}}\right)\left(f^{e}+f^{h}\right) ; \\
& f_{12 ; 3}^{e}=\left(\mathrm{e}^{-s U_{12 ; 3}^{e}}-1\right)\left\langle\theta_{\Delta}\left(\epsilon-\Xi_{12}^{3}+\delta \xi_{1}\right)\right\rangle^{\prime}, \\
& f_{12 ; 3}^{h}=\left(\mathrm{e}^{-2 s U_{12 ; 3}^{h}}-1\right)\left\langle\theta_{\Delta}\left(\epsilon-\Xi_{12}^{3}-\delta \xi_{3}\right)\right\rangle^{\prime},
\end{aligned}
$$

where $\langle\ldots\rangle^{\prime}$ denotes the average (73) with levels $1,2,3$ excluded, and the potentials $U_{12 ; 3}^{(e, h)}$ were defined in Eq. (115). Using the fact that $\delta \xi_{1}\left(\epsilon-\Xi_{l_{1} l_{2}}^{l_{3}}\right)$ from Eq. (161a) is not singular at $\Xi_{12}^{3} \rightarrow \epsilon$ and $|\delta \xi| \ll \Delta$, we can perform the integration in the same way as in Sec 6.4 to obtain

$$
\ln W_{3}^{(1,0)}=-\left(\pi s \Gamma^{(0,0)}\right)^{1 / 2} \frac{6 \lambda M T}{\delta_{\zeta}}
$$

Here the only effect of the shifts was to make the electron contribution statistically independent from the hole one. As a result, the cross-term in Eq. (116b) vanishes and the numerical coefficient is changed in comparison with Eq. (117).

Let us now consider the modification of the fourth order result (123). After two iterations of Eq. (161b), see also Fig. 14 and Eq. (162), we find

$$
\begin{aligned}
\Gamma^{(2,0)} & =\Gamma^{(0,0)} \lambda^{4} \delta_{\zeta}^{4} \sum_{l_{1}, l_{2} ; l_{3}} F_{l_{1}, l_{2} ; l_{3}} \sum_{l_{4}, l_{5} ; l_{6}}{ }^{\prime} F_{l_{4}, l_{5} ; l_{6}}^{\Rightarrow} \\
& \times\left\{\frac{2 Y_{l_{1}, l_{2}}^{l_{3}, l} \theta_{\Delta}\left(\epsilon-\Xi_{l_{1} l_{2}}^{l_{3}}\right)}{\left[\epsilon-\Xi_{l_{1} l_{2}}^{l_{3}}-\delta \xi_{l_{1}}\left(\epsilon-\Xi_{l_{2}}^{l_{3}}\right)\right]^{2}} \frac{2 Y_{l_{4}, l_{5}}^{l_{1}, l_{6}} \theta_{\Delta}\left(\epsilon-\Xi_{l_{2} l_{4} l_{5}}^{l_{3} l_{6}}\right)}{\left[\epsilon-\Xi_{l_{2} l_{4} l_{5}}^{l_{3} l_{6}}-\delta \xi_{l_{5}}\left(\epsilon-\Xi_{l_{2} l_{4}}^{l_{3} l_{6}}\right)\right]^{2}}\right. \\
& +\frac{2 Y_{l_{1}, l_{2}}^{l_{3}, l} \theta_{\Delta}\left(\epsilon-\Xi_{l_{1} l_{2}}^{l_{3}}\right)}{\left[\epsilon-\Xi_{l_{1} l_{2}}^{l_{3}}-\delta \xi_{l_{1}}\left(\epsilon-\Xi_{l_{2}}^{l_{3}}\right)\right]^{2}} \frac{Y_{l_{4}, l_{5}}^{l_{1}, l_{6}} \theta_{\Delta}\left(\epsilon-\Xi_{l_{1} l_{2} l_{6}}^{l_{4} l_{5}}\right)}{\left[\epsilon-\Xi_{l_{2} l_{4} l_{5}}^{l_{3} l_{6}}+\delta \xi_{l_{6}}\left(\Xi_{l_{2} l_{4} l_{5}}^{l_{3}}-\epsilon\right)\right]^{2}} \\
& +\frac{Y_{l_{1}, l_{2}}^{l_{3}, l} \theta_{\Delta}\left(\epsilon-\Xi_{l_{1} l_{2}}^{l_{3}}\right)}{\left[\epsilon-\Xi_{l_{1} l_{2}}^{l_{3}}+\delta \xi_{l_{3}}\left(\Xi_{l_{1} l_{2}}-\epsilon\right)\right]^{2}} \frac{2 Y_{l_{3}, l_{6}}^{l_{4}, l_{5}} \theta_{\Delta}\left(\epsilon-\Xi_{l_{1} l_{2} l_{6}}^{l_{4} l_{5}}\right)}{\left[\epsilon-\Xi_{l_{1} l_{2} l_{6}}^{l_{4} l_{5}}+\delta \xi_{l_{4}}\left(\Xi_{l_{1} l_{2} l_{6}}^{l_{5}}-\epsilon\right)\right]^{2}} \\
& \left.+\frac{Y_{l_{1}, l_{2}}^{l_{3}, l} \theta_{\Delta}\left(\epsilon-\Xi_{l_{1} l_{2}}^{l_{3}}\right)}{\left[\epsilon-\Xi_{l_{1} l_{2}}^{l_{3}}+\delta \xi_{l_{3}}\left(\Xi_{l_{1} l_{2}}-\epsilon\right)\right]^{2}} \frac{Y_{l_{3}, l_{6}}^{l_{4}, l_{5}} \theta_{\Delta}\left(\epsilon-\Xi_{l_{1} l_{2} l_{6}}^{l_{4} l_{5}}\right)}{\left[\epsilon-\Xi_{l_{1} l_{2} l_{6}}^{l_{4} l_{5}}-\delta \xi_{l_{6}}\left(\epsilon-\Xi_{l_{1} l_{2}}^{l_{4} l_{5}}\right)\right]^{2}}\right\}
\end{aligned}
$$

where the prime in the second sum has the same meaning as in Eq. (123). Each term in Eq. (166) produces its own cluster function, see Eq. (125). Similarly 


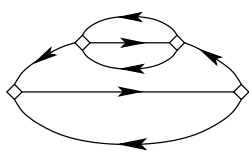

$(\mathrm{a} 0)$

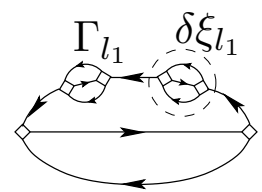

(a1)

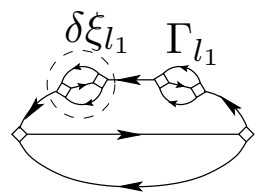

(a2)

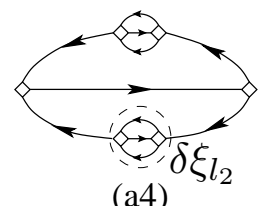

(a4)

(a6)

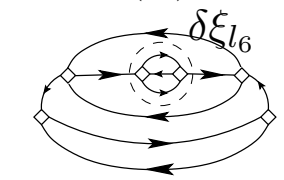

(b)

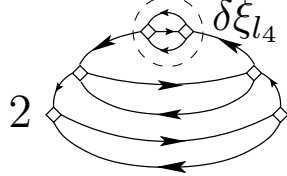

(a5)

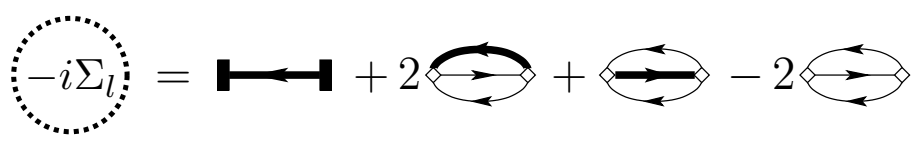

Fig. 23. (a) Including the level shift into the fourth order ImSCBA diagram. Only diagrams a.1 and a. 2 are resonant, i.e., divergences in $\delta \xi_{l}$ and $\Gamma_{l}$ are correlated. (b) Linearized SCBA approximation which incorporates the simultaneous divergences in $\delta \xi_{l}$ and $\Gamma_{l}$.

to Eq. (164), the cross-correlation terms vanish and we obtain after obvious shifts of the variables

$$
\begin{gathered}
\ln W^{(2,0)}=\prod_{l=1}^{6}\left(\int \frac{\mathrm{d} \xi_{l}}{\delta_{\zeta}} \sum_{n_{l}= \pm 1} \frac{\exp \frac{n_{l} \xi_{l}}{2 T}}{2 \cosh \frac{\xi_{l}}{2 T}}\right) \tilde{f}\left(\begin{array}{c}
12 ; 3 \\
45 ; 6
\end{array}\right) ; \\
\tilde{f}\left(\begin{array}{c}
12 ; 3 \\
45 ; 6
\end{array}\right)=f^{e e}\left(\begin{array}{c}
12 ; 3 \\
45 ; 6
\end{array}\right)+\frac{1}{2} f^{e h}\left(\begin{array}{c}
12 ; 3 \\
45 ; 6
\end{array}\right)+\frac{1}{2} f^{h e}\left(\begin{array}{c}
12 ; 3 \\
45 ; 6
\end{array}\right)+\frac{1}{4} f^{h e}\left(\begin{array}{c}
12 ; 3 \\
45 ; 6
\end{array}\right) ; \quad(167 \mathrm{~b}) \\
f^{e e}=\left(\mathrm{e}^{-s U^{e e}}-1\right)\left\langle\theta_{\Delta}\left[\epsilon-\Xi_{12}^{3}+\delta \xi_{1}\left(\epsilon-\Xi_{2}^{3}\right)\right] \theta_{\Delta}\left[\epsilon-\Xi_{245}^{36}+\delta \xi_{5}\left(\epsilon-\Xi_{24}^{36}\right)\right]\right\rangle^{\prime} ; \\
f^{e h}=\left(\mathrm{e}^{-2 s U^{e h}}-1\right)\left\langle\theta_{\Delta}\left[\epsilon-\Xi_{12}^{3}+\delta \xi_{1}\left(\epsilon-\Xi_{2}^{3}\right)\right] \theta_{\Delta}\left[\epsilon-\Xi_{245}^{36}-\delta \xi_{6}\left(\Xi_{245}^{3}-\epsilon\right)\right]\right\rangle^{\prime} ; \\
f^{h e}=\left(\mathrm{e}^{-2 s U^{h e}}-1\right)\left\langle\theta_{\Delta}\left[\epsilon-\Xi_{12}^{3}-\delta \xi_{1}\left(\Xi_{12}-\epsilon\right)\right] \theta_{\Delta}\left[\epsilon-\Xi_{45}^{126}-\delta \xi_{4}\left(\Xi_{126}^{5}-\epsilon\right)\right]\right\rangle^{\prime} ; \\
f^{h h}=\left(\mathrm{e}^{-4 s U^{h h}}-1\right)\left\langle\theta_{\Delta}\left[\epsilon-\Xi_{12}^{3}-\delta \xi_{1}\left(\Xi_{12}-\epsilon\right)\right] \theta_{\Delta}\left[\epsilon-\Xi_{126}^{45}+\delta \xi_{6}\left(\epsilon-\Xi_{12}^{45}\right)\right]\right\rangle^{\prime},
\end{gathered}
$$

where $\langle\ldots\rangle^{\prime}$ denotes averaging (73) with the levels $1,2,3,4,5,6$ excluded. The potentials $U$ here are defined in Eq. (124). 
Apparently, the role of the level shifts in Eqs. (167c) is similar to that in Eq. (164) - perturbative modification of the cut-off. However, unlike the lowest order perturbative corrections, the shifts in Eq. (167c) contain a resonant term which depends on variables $\xi_{1}, \ldots, \xi_{6}$ only and therefore can not be treated as a non-correlated random number. To see the origin of such resonant term, consider the argument of the first $\theta$-function in $f^{e e}$. From Eq. (161a) with $I=0$, we find after the same variable shifts as in $f^{e e}$

$$
\delta \xi_{1}\left(\epsilon-\Xi_{2}^{3}\right)=\frac{2 \lambda^{2} \delta_{\zeta}^{2} Y_{4,5}^{6,1} F_{4,5 ; 6}^{\Rightarrow} \theta_{\Delta}\left[\epsilon-\Xi_{452}^{63}+\ldots\right]}{\epsilon-\Xi_{452}^{63}}+\ldots,
$$

where ... denote the terms which contain extra levels. Those terms are random and can be disregarded. One can check by the same method that the shift $\delta \xi_{5}\left(\epsilon-\Xi_{24}^{36}\right)$ always depends on extra levels and does not produce resonance.

Having in mind that the $\theta$-functions in Eqs. (167c) cut off the logartihmic divergence, we neglect the non-resonant terms, ${ }^{18}$ and simplify Eqs. (167c) as

$$
\begin{aligned}
f^{e e}\left(\begin{array}{c}
12 ; 3 \\
45 ; 6
\end{array}\right) & =\left[\mathrm{e}^{-s U^{e e}\left(\begin{array}{c}
12 ; 3 \\
45 ; 6
\end{array}\right)}-1\right] \theta_{\Delta}\left(\epsilon-\Xi_{12}^{3}+\frac{2 \lambda^{2} \delta_{\zeta}^{2} Y_{4,5}^{6,1}}{\epsilon-\Xi_{452}^{63}}\right) \theta_{\Delta}\left(\epsilon-\Xi_{245}^{36}\right) ; \\
f^{e h}\left(\begin{array}{c}
12 ; 3 \\
45 ; 6
\end{array}\right) & =\left[\mathrm{e}^{-2 s U^{e h}\left(\begin{array}{c}
12 ; 3 \\
45 ; 6
\end{array}\right)}-1\right] \theta_{\Delta}\left(\epsilon-\Xi_{12}^{3}+\frac{2 \lambda^{2} \delta_{\zeta}^{2} Y_{4,5}^{6,1}}{\epsilon-\Xi_{452}^{63}}\right) \theta_{\Delta}\left(\epsilon-\Xi_{245}^{36}\right) ; \\
f^{h e}\left(\begin{array}{c}
12 ; 3 \\
45 ; 6
\end{array}\right) & =\left[\mathrm{e}^{-2 s U^{h e}\left(\begin{array}{c}
12 ; 3 \\
45 ; 6
\end{array}\right)}-1\right] \theta_{\Delta}\left(\epsilon-\Xi_{12}^{3}+\frac{\lambda^{2} \delta_{\zeta}^{2} Y_{3,6}^{4,5}}{\epsilon-\Xi_{45}^{126}}\right) \theta_{\Delta}\left(\epsilon-\Xi_{45}^{126}\right) ; \\
f^{h h}\left(\begin{array}{c}
12 ; 3 \\
45 ; 6
\end{array}\right) & =\left[\mathrm{e}^{-4 s U^{h h}\left(\begin{array}{c}
12 ; 3 \\
45 ; 6
\end{array}\right)}-1\right] \theta_{\Delta}\left(\epsilon-\Xi_{12}^{3}+\frac{\lambda^{2} \delta_{\zeta}^{2} Y_{3,6}^{4,5}}{\epsilon-\Xi_{45}^{126}}\right) \theta_{\Delta}\left(\epsilon-\Xi_{45}^{126}\right)
\end{aligned}
$$

Substituting Eq. (169) into Eqs. (167a)-(167b) and performing integration we obtain analogously to Eq. (127)

$$
\ln W^{(2,0)}=-\left(\pi s \Gamma^{(0,0)}\right)^{1 / 2}\left[\frac{6 \lambda M T}{\delta_{\zeta}}\right]^{2} \ln \left(\frac{\Delta^{2}}{\lambda^{2} \delta_{\zeta}^{2}}\right) .
$$

In addition to the change in the overall numerical factor similar to Eq. (165), Eq. (170) shows new important feature. Namely, the argument of the logartihm is no longer dependent on the parameter $s$, or, in other words, the algebraic tail in the distribution function is suppressed in comparison with Eq. (127). This is the manifestation of the level repulsion in complete analogy with the arguments of Anderson [1].

\footnotetext{
${ }^{18}$ Keeping them will be beyond the accuracy of LSCBA approximations where the terms of the same order were neglected in the very beginning.
} 


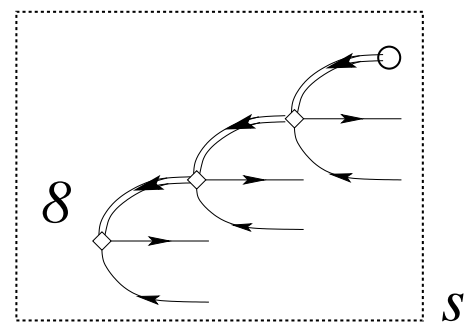

Fig. 24. The cluster function $f^{e e e}$ for the six order perturbation theory expansion. The notation is defined on Figs. 11, 12 and in Eq. (162).

The procedure outlined above can be continued to all orders of perturbation theory. In particular, for the sixth order cluster function, Fig. 24, one finds

$$
\begin{aligned}
f^{e e e}= & {\left[\mathrm{e}^{-s U^{e e e}\left(\begin{array}{c}
12 ; 3 \\
45 ; 6 \\
78 ; 9
\end{array}\right)}-1\right] \theta_{\Delta}\left(\epsilon-\Xi_{12}^{3}+\frac{2 \lambda^{2} \delta_{\zeta}^{2} Y_{4,5}^{6,1}}{\epsilon-\Xi_{245}^{36}}\right) } \\
& \times \theta_{\Delta}\left(\epsilon-\Xi_{245}^{36}+\frac{2 \lambda^{2} \delta_{\zeta}^{2} Y_{7,8}^{9,4}}{\epsilon-\Xi_{2578}^{369}}\right) \theta_{\Delta}\left(\epsilon-\Xi_{2578}^{369}\right) ; \\
U^{\text {eee }}= & \frac{8 \Gamma^{(0,0)} \lambda^{6} \delta_{\zeta}^{6} Y_{1,2}^{3, l} F_{1,2 ; 3}^{\Rightarrow} Y_{4,5}^{6,1} F_{4,5 ; 6} Y_{7,8}^{9,4} F_{7,8 ; 9}^{\Rightarrow}}{\left(\epsilon-\Xi_{12}^{3}\right)^{2}\left(\epsilon-\Xi_{245}^{36}\right)^{2}\left(\epsilon-\Xi_{2578}^{369}\right)^{2}}
\end{aligned}
$$

which means that the scale of the integration is determined by the energy of the previous generation only. This transfer matrix structure of the cluster functions repeats itself in all orders and makes it possible to perform the integration in any order. Instead of Eq. (135) we find

$$
\begin{aligned}
& \ln W^{(n, m)}=-\left(s \Gamma^{(0,0)}\right)^{1 / 2} \mathcal{C}(n, m) \\
& \quad \times\left[\frac{6 \lambda M T}{\delta_{\zeta}} \ln \left(\frac{\Delta^{2}}{\delta_{\zeta}^{2} \lambda^{2}}\right)\right]^{n}\left[I \ln \left(\frac{\Delta^{2}}{\delta_{\zeta}^{2} I^{2}}\right)\right]^{m},
\end{aligned}
$$

i.e., once again the algebraic tail becomes "more short-range" due to the suppression of the large denominators. Coefficients $\mathcal{C}(n, m)$ are insensitive to the resonant denominators and they are still given by Eq. (136). We, therefore, can repeat all of the arguments after Eq. (136) and obtain Eq. (160) after replacement (128).

To conclude, the level repulsion present in SCBA but neglected in SCBA leads to the change in the numerical prefactor in the expression for the transition temperature [cf. Eq. (160) with Eq. (140)] but does not affect the statement about the existence of the transition itself. All other corrections lead to the perturbative corrections to $T_{c}$. 


\subsection{Validity of ImSCBA in the metallic phase}

In the metallic phase the classification of non-SCBA diagrams into interaction vertex corrections and interference terms is the same as presented in Secs. 7.1 and 7.2 for the insulating phase. What changes is that the resonant terms in all of the expressions now acquire the finite width $\Gamma^{(i n)}$

$$
\pi \delta(x)+P \frac{\Gamma}{x^{2}} \rightarrow \frac{\Gamma^{(i n)}}{x^{2}+\left[\Gamma^{(i n)}\right]^{2}} ; \quad P \frac{1}{x} \rightarrow \frac{x}{x^{2}+\left[\Gamma^{(i n)}\right]^{2}}
$$

where $\Gamma^{(i n)}$ is given by Eq. (74). This finite width prohibits vanishing of the denominators, thereby cutting off the power-law tails of the distribution functions for all the quantities considered in Secs. 7.1-7.3. As a result, all the cumulants of the distribution function become finite. Moreover, under the condition (80) the distribution functions may be considered Gaussian.

Another feature is that the level occupation (50) is no longer binary, $n_{l}(\epsilon)=$ \pm 1 , as in insulating phase, but it is kept close to its equlibrium value $n_{l}(\epsilon)=$ tanh $\frac{\epsilon}{2 T}$ by the energy relaxation. This further suppresses the fluctuations by the factor of $\delta_{\zeta} / T$.

On the other hand, in Sec. 7.1, we established that the role of the higher-order correction to the vertices is the perturbative renormalization of the SCBA results. As the scale of the fluctuation of those vertices is suppressed even further in the metallic phase, neglecting this renormalization is justified even more in this phase.

The role of the particle permutations and interference in the final state, see Sec. 7.2 is investigated by direct evaluation of the mesoscopic fluctuations and averages of the diagrams of Fig. 19 in a fashion of Fig. 8. Those contribution are smaller than SCBA values by a factor of $M$ at least. Therefore, deep in the metallic phase the kinetic equation consideration of transport in Sec. 5.2 is well justified.

Finally, in the insulating phase the most important effect, not included in ImSCBA, was many-mody level repulsion, see discussion around Eq. (159). The energy scale of this repulsion is $\lambda \delta_{\zeta}$. On the other hand, at $T>T_{i n}$, where $T_{\text {in }}$ is defined in Eq. (80), we find $\Gamma^{(i n)}>\lambda \delta_{\zeta}$. This means that the spectrum structure produced by this repulsion is smeared by inelastic processes and need not be taken into account.

Thus, we conclude that the ImSCBA is justified parametrically in deep in the metallic phase. 


\section{Conclusions}

This paper is devoted to the analysis of the low-temperature transport in disordered conductors with localized one-particle states. The main question is whether the electron-electron interaction alone is sufficient to establish the thermal equilibrium in the system. The same question can be formulated even more boldly - whether there is a many-body mobility threshold, i.e., energy separating the many-body states localized in the Fock space of the system from the states which are delocalized.

One can apply weak-localization arguments to show that conductivity at high enough temperatures is non-zero. It is not disputable as long as the inelastic dephasing rate $1 / \tau_{\phi}$ exceeds the level spacing in one-particle localization volume (cell) $\delta_{\zeta}$. Extension of this approach to lower temperatures is problematic as the quantum corrections to conductivity diverge. For this reason, in order to describe the low-temperature behavior, we adopt a different strategy. We formulate the effective Hamiltonian description for the processes with the energy transfer of the order of $\delta_{\zeta}$. Reduction of the original Hamiltonian to the effective one is not performed systematically. Nevertheless, we believe that it is an apropriate low-energy limit of the theory of electrons in disorder potential.

Statisitical analysis based on the effective Hamiltonian enables us to demonstrate the stability of two qualitatively different phases - metallic, for $T>T_{c}$ and insulating for $T<T_{c}$, where $T_{c}$ is given by Eq. (160). This corresponds to the existence of the many-body mobility threshold $\mathcal{E}_{c}$ related to $T_{c}$ by the thermodynamic formula (101).

We show that deep in the metallic phase, $T \gg T_{c}$ (see Sec. 5) the transport coefficients in the system are self-averaging. Using this fact, we derived the quantum Boltzman equation. The temperature dependence of the electrical conductivity $\sigma(T)$, following from this equation (see Sec. 5.2), is quite non-trivial even for $T \gg T_{c}$. Namely, $\sigma(T)$ increases with $T$ as $T^{2}$ at low temperatures, while at high temperatures it saturates, approaching the Drude limit. Thermal conductivity deviates from the Wiedemann-Franz law with the decreasing temperature. However, this deviation is never strong, see Fig. 10.

In the insulating phase, $T<T_{c}$, we use Feynmann diagram technique to determine the probability distribution function for quantum-mechanical transition rates. The probability of an escape rate from a given quantum state to be finite turns out to vanish in every order of the perturbation theory in electron-electron interaction. Thus, in the absence of coupling to any external bath (phonons) electron-electron interaction alone is unable to cause the relaxation and establish the thermal equilibrium. In other words, the insulating phase is stable. 
Although $\sigma(T)=0$ exactly as long as $T<T_{c}$, the stability of the insulator decreases as $T$ approaches $T_{c}$. It means that effects of interaction of the electrons with the external bath (phonons) become more and more pronounced. More precisely, if the electron-phonon coupling is weak and $T \ll T_{c}$, one phonon can cause at most one electron to hop because the phase volume of the accessible final states is quite small. The closer is $T$ to $T_{c}$, the bigger is this phase volume, and in the vicinity of the transition point one phonon can initiate a whole cascade of the electronic hops. The size of the cascades fluctuates strongly, depending on the realization of disorder in the system. The typical size of the cascade grows at $T \rightarrow T_{c}$ [see Eq. (142)]. It means, that even the infinitesimal electron-phonon interaction would produce a finite conductivity. This is the onset of the metallic phase.

We also conclude that the phonon-induced hopping conductivity in the insulating phase close to the transition is strongly enhanced by the electronelectron interaction. This conclusion can be relevant for the numerous experiments [32-36], where the observed conductivity in the strongly localized phase of disordered conductors was too large to be explained by conventional theory of phonon-assisted hopping conductivity.

It should be emphasized that the many-body localization, which we discuss in this paper, is qualitatively different from conventional finite temperature Metal to Insulator transitions, such as formation of a band insulator due to the structural phase transition or Mott-Hubbard transition [37,38]. In these two cases, at a certain temperature $T^{*}$ a gap appears in the spectrum of charge excitation (Mott insulator) or all excitations (band insulator). However the conductivity remains finite although exponentially small as long as $T>0$. This is not the case for many-body localization, which causes exactly zero conductivity in the low-temperature phase.

Is the many-body localization a true thermodynamical phase transition with corresponding singularities in all equilibrium properties? This question definitely requires additional studies, however, some speculations can be put forward. The physics described in the present paper is associated with the change of the characteristics of the many-body wavefunctions. It is well known that for non-interacting systems localization-delocalization transition does not influence the average density of states, i.e., it does not affect any macroscopic thermodynamic properties. Application of the same logic to the exact manybody eigenvalues would indicate that the many-body localization tranisition is not followed by any singularities in the static specific heat, etc. On the other hand, at this point we can not rule out the possibility that this conclusion is an artefact of treating the real parts of the electron self-energies with an insufficient accuracy. Most likely scenario, to our opinion, is that the insulating phase behaves like a glass (spin or structural) and demonstrates all the glassy properties [19], like absence of ergodicity (even when some coupling 
with phonons is included), effects of aging, etc. Question of the equilibrium susceptibilities in the latter case becomes quite meaningless.

The quantitative theory built in this paper assumes that the interaction is weak. On the other hand, qualitative consideration of the localization of manybody excitations does not rely on this assumption. The important ingredients are (i) localization of single-particle excitations, and (ii) Fermi statistics. Consider, as an example, Wigner crystal [39]. It is well known that strong enough interaction leads to a spontaneous breaking of the translational symmetry in $d$-dimensional clean systems at $d \geq 2$. In a clean system Wigner crystallization is either a first-order phase transition $(d=3)$, or a Kosterlitz-Thouless transition $(d=2)$. Even weak disorder destroys both translational and orientational order [40] and pins the crystal. The symmetry of this state is thus not different from the symmetry of a liquid, and the thermodynamic phase transition is commonly believed to be reduced to a crossover.

We argue that the many-body localization provides the correct scenario for the finite-temperature "melting" transition between the insulating phase, which may be called "solid", and the metallic phase, which may be called "liquid". Indeed, the conductivity of the pinned Wigner crystal is provided by the motion of defects. At low temperatures and in the absence of the external bath, all defects are localized by the one-particle Anderson mechanism. Phonon modes of the Wigner crystal are localized as well, so the system should behave as a many-body insulator. As the temperature is increased, the many-body metalinsulator transition occurs, though it is not clear at present, whether it occurs before or after the crystalline order is destroyed at distances smaller than Larkin's scale. Construction of effective theory of such a transition is a problem which deserves further investigation.

\section{Acknowledgements}

We are grateful to M. E. Gershenson and V. E. Kravtsov for valuable remarks. We also want to thank A. D. Mirlin for clarifying statements made in their preprint [28] and revealing the content of Eqs. (19)-(20) of Ref. [28] to us.

I.L.A. was supported by Packard foundation, B.L.A. acknowledges financial support from US DOE Office of Science under contract No. W-31-109-ENG38, ARO/ARDA (DAAD19-02-1-0039) DARPA under QuIST program and NSF grant DMR 0210575.

Note added- When the current manuscript was almost completed, a new version of preprint [28] appeared [41], which deals with some of the problems constituting the content of the present paper. 


\section{A Slow energy relaxation?}

Let us now come back to the assumption of the strong inelastic relaxation which was used to validate expansion (91). One could think that in some cases this assumption is not valid, which would lead to the deviation of the temperature dependences from those of Eqs. (93). To investigate the limits of validity of Eqs. (93)-(100), we employ the following qualitative arguments. These arguments only slightly modify the discussion after Eq. (99).

We notice that the transport still occurs through rare pin-holes. However, the insufficient rate of the inelastic processes allows the electron to explore an energy strip of the width $\bar{\epsilon} \ll T$ before leaving the site via tunneling to the neighboring sites. Correspondingly, instead of using Eq. (91) one may look for the solution in the form

$$
\Phi(\epsilon, \boldsymbol{\rho} ; t)=\frac{\delta \mu(\boldsymbol{\rho}, t)}{\bar{\epsilon}} \tilde{\beta}(\epsilon / \bar{\epsilon}), \quad \int \mathrm{d} x \tilde{\beta}(x) \simeq 1,
$$

where $\bar{\epsilon}$ is a scale to be found self-consistently. The conductivities are still given by Eqs. (93), but the random quantities $\mathcal{B}_{\sigma}, \mathcal{B}_{\kappa}$ replaced by different ones $\tilde{\mathcal{B}}_{\sigma}, \tilde{\mathcal{B}}_{\kappa}$, which are defined through $\tilde{\beta}(x T / \bar{\epsilon})$ rather than through $\beta_{\sigma, \kappa}(x)$. Thus, either a straightforward calculation, or a qualitative argument, similar to that after Eq. (99), give for the typical $\tilde{\mathcal{B}}$ an estimate

$$
\tilde{\mathcal{B}} \simeq \frac{\Gamma^{(i n)} \bar{\epsilon}}{\delta_{\zeta}^{2}}
$$

valid if $\tilde{\mathcal{B}} \ll 1$. To find $\bar{\epsilon}$, we balance the elastic and inelastic terms in Eq. (90). The estimate for the elastic term is

$$
I^{2} \delta^{3} \sum_{\boldsymbol{a}}\langle A(\epsilon, \boldsymbol{\rho}) A(\epsilon, \boldsymbol{\rho}+\boldsymbol{a})\rangle \Phi \simeq I^{2} \delta_{\zeta} \tilde{\mathcal{B}} \Phi \simeq \Gamma^{(i n)} \frac{I^{2} \bar{\epsilon}}{\delta_{\zeta}} \Phi,
$$

while the inelastic term is estimated from Eq. (87e) as:

$$
\widehat{\mathrm{St}}_{\Phi} \Phi \simeq D^{(\epsilon)} \partial_{\epsilon}^{2} \Phi \simeq \Gamma^{(i n)} \frac{\delta_{\zeta}^{2} M^{2}}{\bar{\epsilon}^{2}} \Phi
$$

Requiring these two rates to be of the same order, we estimate the scale $\bar{\epsilon}$ as

$$
\bar{\epsilon}=\delta_{\zeta}\left(\frac{M}{I}\right)^{2 / 3}
$$

Conditions $\bar{\epsilon} \ll T$ and $\tilde{\mathcal{B}} \ll 1$ would produce a parametric temperature region

$$
\delta_{\zeta}\left(\frac{M}{I}\right)^{2 / 3} \lesssim T \lesssim \frac{\delta_{\zeta}}{\lambda^{2} M}\left(\frac{I}{M}\right)^{2 / 3}
$$


in which the non-equilibrium function $\Phi(\epsilon)$ does not have the quasi-equilibrium shape (91). However, existence of such a regime requires the condition

$$
\left(\frac{I}{\lambda}\right)^{4 / 3}\left(\frac{1}{\lambda M^{2}}\right)^{7 / 6} \lambda^{1 / 2} \gg 1
$$

which is not consistent with the additional assumptions $M^{2} \lambda \simeq 1, I \simeq \lambda \ll 1$. Therefore, the regime of the slow energy relaxation is not feasible for this model and Eqs. (93), (94) describe the entire temperature dependence for $T \gtrsim T_{\text {in }}$.

\section{B Probability distributions for $\mathcal{B}_{\sigma, \kappa}$.}

Let us represent the definitions (92c) in the form

$$
\mathcal{B}=\sum_{l, l^{\prime}} B\left(\xi_{l}, \xi_{l^{\prime}}\right)=\sum_{l, l^{\prime}} \frac{\delta_{\zeta}}{2 T} \beta\left(\frac{\xi_{l}}{2 T}\right) \frac{2 \delta_{\zeta} \Gamma / \pi}{\left(\xi_{l}-\xi_{l^{\prime}}^{\prime}\right)^{2}+4 \Gamma^{2}}
$$

where the functions $\beta_{\sigma}(x)$ and $\beta_{\kappa}(x)$ are given by Eq. (92d). (We omit the superscript "(in $)$ " of $\Gamma^{(i n)}$ everywhere in this appendix). The positions of the levels $\xi_{l}, \xi_{l^{\prime}}^{\prime}$ are assumed to be completely uncorrelated, so the sought characteristic function $\tilde{P}(s)=\left\langle\mathrm{e}^{-s \mathcal{B}}\right\rangle$ can be represented as

$$
\tilde{P}(s)=\lim _{N \rightarrow \infty}\left(\int_{-N \delta_{\zeta} / 2}^{N \delta_{\zeta} / 2} \prod_{l=1}^{N} \frac{\mathrm{d} \xi_{l}^{+} \mathrm{d} \xi_{l}^{-}}{\left(N \delta_{\zeta}\right)^{2}}\right) \mathrm{e}^{-s \sum_{l, l^{\prime}=1}^{N} B\left(\xi_{l}^{+}, \xi_{l^{\prime}}^{-}\right)}
$$

Equation is equivalent to the partition function of the classical gas of two species, $( \pm)$, and interacting to each other via pair potential $B\left(\xi_{l}^{+}, \xi_{l^{\prime}}^{-}\right)$. It can be immediately evaluated using Mayer-Mayer cluster expansion [29]. We will keep the contributions upto four-particle clusters to justify the further approximations:

$$
\begin{aligned}
& \ln \tilde{P}(s)=\ln \tilde{P}^{(2)}(s)+\ln \tilde{P}^{(3)}(s)+\ln \tilde{P}^{(4)}(s) \ldots \\
& \ln \tilde{P}^{(2)}(s)=\int_{-\infty}^{\infty} \frac{\mathrm{d} \xi_{1} \mathrm{~d} \xi_{2}}{\delta_{\zeta}^{2}} f_{12} ; \\
& \ln \tilde{P}^{(3)}(s)=\frac{1}{2} \int_{-\infty}^{\infty} \frac{\mathrm{d} \xi_{1} \mathrm{~d} \xi_{2} \mathrm{~d} \xi_{3}}{\delta_{\zeta}^{3}}\left[f_{12} f_{32}+f_{21} f_{23}\right] \\
& \ln \tilde{P}^{(4)}(s)=\int_{-\infty}^{\infty} \frac{\mathrm{d} \xi_{1} \ldots \mathrm{d} \xi_{4}}{\delta_{\zeta}^{3}}\left[f_{12} f_{32} f_{34}+\frac{f_{12} f_{32} f_{34} f_{14}}{2}\right],
\end{aligned}
$$


where

$$
f_{12}=\exp \left[-s B\left(\xi_{1}, \xi_{2}\right)\right]-1
$$

is the Mayer's $f$-function which vanishes rapidly with the distance between the levels $\left|\xi_{1}-\xi_{2}\right|$. Performing integrations in Eqs. (B.3b)-(B.3d) with the help of definition (B.1) and using $\Gamma \gg T$, we find

$$
\begin{aligned}
& \ln \tilde{P}^{(2)}(s)=-\int_{-\infty}^{\infty} \mathrm{d} x\left[r S_{2}\left(\frac{s \beta(x)}{r}\right)\right] ; \\
& \ln \tilde{P}^{(3)}(s)=\frac{\delta_{\zeta}}{2 T} \int_{-\infty}^{\infty} \mathrm{d} x\left[r S_{2}\left(\frac{s \beta(x)}{r}\right)\right]^{2} ; \\
& \ln \tilde{P}^{(4)}(s)=\left(\frac{\delta_{\zeta}}{2 T}\right)^{2} r^{3} \int_{-\infty}^{\infty} \mathrm{d} x\left[-S_{2}^{3}\left(\frac{s \beta(x)}{r}\right)+S_{4}\left(\frac{s \beta(x)}{r}\right)\right] ; \\
& S_{2}(y)=y \mathrm{e}^{-y}\left[\mathcal{I}_{0}(y)+\mathcal{I}_{1}(y)\right] . \\
& S_{4}(y)=\int_{-\infty}^{\infty} \frac{\mathrm{d} x_{1} \mathrm{~d} x_{2} \mathrm{~d} x_{3}}{(2 \pi)^{3}} \\
& \quad \times R\left(y, x_{2}\right) R\left(y, x_{2}-x_{3}\right) R\left(y, x_{3}-x_{4}\right) R\left(y, x_{4}\right) \\
& R(y ; x)=\exp \left(-\frac{2 y}{1+x^{2}}\right)-1 .
\end{aligned}
$$

Here $\mathcal{I}_{0}$ and $\mathcal{I}_{1}$ are the modified Bessel functions. We also have introduced the parameter $r \equiv 8 \pi \Gamma T / \delta_{\zeta}^{2}$, see Eq. (96).

Next step is to notice that $T \gg \delta_{\zeta}$ and the observable quantities are contributed by $s$ such as $r S_{2}(s / r) \lesssim 1$. Therefore, the contribution from the higher cluster are suppressed and can be neglected. All the further calculation is performed using $\tilde{P}^{(2)}$ only.

Two limiting cases can be considered. First, if $r \gg 1, S(x)$ can be expanded $S(x)=x(1-x / 2+\ldots)$, which gives

$$
\begin{array}{r}
r \gg 1:\left\{\begin{array}{c}
\ln \tilde{P}_{\sigma}(s) \\
\ln \tilde{P}_{\kappa}(s)
\end{array}\right\} \approx-s+\frac{s^{2}}{2 r} \int_{-\infty}^{\infty} \mathrm{d} x\left\{\begin{array}{c}
\beta_{\sigma}^{2}(x) \\
\beta_{\kappa}^{2}(x)
\end{array}\right\} \\
=-s+\frac{s^{2}}{2 r}\left\{\begin{array}{c}
1 / 3 \\
7 / 5-12 / \pi^{2}
\end{array}\right\} .
\end{array}
$$

In the opposite case, $r \ll 1$, we use the asymptotic expansions of the Bessel 
functions to approximate $S(x) \approx \sqrt{2 /(\pi x)}$, to obtain

$$
\begin{array}{r}
r \ll 1: \quad\left\{\begin{array}{l}
\ln \tilde{P}_{\sigma}(s) \\
\ln \tilde{P}_{\kappa}(s)
\end{array}\right\} \approx-\sqrt{\frac{2}{\pi} r s} \int_{-\infty}^{\infty} \mathrm{d} x\left\{\begin{array}{c}
\sqrt{\beta_{\sigma}(x)} \\
\sqrt{\beta_{\kappa}(x)}
\end{array}\right\} \\
=-\sqrt{\frac{2}{\pi} r s}\left\{\begin{array}{c}
\pi / \sqrt{2} \\
4 \sqrt{6} \mathrm{G} / \pi
\end{array}\right\} \equiv\left\{\begin{array}{c}
-\sqrt{c_{\sigma} r s} \\
-\sqrt{c_{\kappa} r s}
\end{array}\right\},
\end{array}
$$

where $\mathrm{G} \approx 0.916$ is the Catalan's constant.

It is instructive to calculate the inverse Laplace transform of $\tilde{P}_{\sigma, \kappa}(s)$ and find the distributions functions $P_{\sigma, \kappa}(\mathcal{B})$. In both limiting cases of large and small $r$ one can use the saddle point approximation. For $r \gg 1$ the distributions are approximately Gaussian with $\left\langle\mathcal{B}_{\sigma, \kappa}\right\rangle=1$ and

$$
\left\langle\mathcal{B}_{\sigma}^{2}\right\rangle-\left\langle\mathcal{B}_{\sigma}\right\rangle^{2}=\frac{1}{3 r}, \quad\left\langle\mathcal{B}_{\kappa}^{2}\right\rangle-\left\langle\mathcal{B}_{\kappa}\right\rangle^{2}=\frac{7 / 5-12 / \pi^{2}}{r},
$$

corresponding to self-averaging of $\mathcal{B}_{\sigma, \kappa}$. For $r \ll 1$ have:

$$
P(\mathcal{B})=\int_{-i \infty}^{i \infty} \frac{\mathrm{d} s}{2 \pi i} \mathrm{e}^{s \mathcal{B}-\sqrt{c r s}} \approx \frac{\sqrt{c r / \pi}}{2 \mathcal{B}^{3 / 2}} \mathrm{e}^{-c r /(4 \mathcal{B})}
$$

It is noteworthy that, for $\mathcal{B} \gg r$ this ditribution coincides with the distribution of the largest of $n=\sqrt{8 \pi c}\left(T / \delta_{\zeta}\right)$ independent random variables

$$
\frac{\delta_{\zeta}}{4 T} \frac{2 \delta_{\zeta} \Gamma / \pi}{\xi_{1}^{2}}, \ldots, \frac{\delta_{\zeta}}{4 T} \frac{2 \delta_{\zeta} \Gamma / \pi}{\xi_{n}^{2}}
$$

[see Eq. (92c)], with $\xi_{i}$ uniformly distributed in the range $0<\xi_{i}<\delta_{\zeta} / 2$.

\section{Cancellation of the cutoff $\Delta$}

The formal reason to introduce the cutoff $\Delta$ in Sec. 6 was the insufficient ability of the $3 n$-particle potentials to confine particles, which resulted in logarithmic divergences in the leading terms of the cluster expansion for $n>1$. On the other hand, since the transition is associated with anomalously small values of the energy denominators, rather than anomalously large ones, it is natural to expect the transition condition not to contain $\Delta$ at all. This means that $\Delta$ should cancel out when higher-order terms of the cluster expansion are taken into account. Moreover, this cancellation should occur for each component of the potential separately (e.g., for each of the 6-particle potentials $U^{e e}, U^{e h}$, 

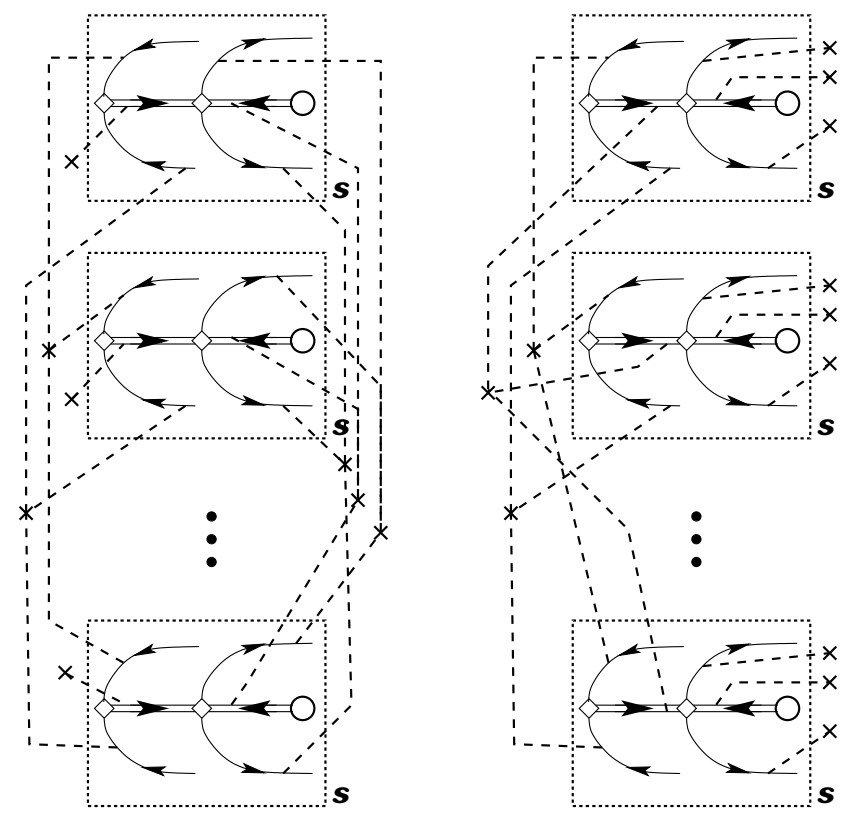

Fig. C.1. The diagrams canceling the cutoff $\Delta$ in the fourth diagram of Fig. 14 .

$U^{h e}$, and $\left.U^{h h}\right)$. In this appendix we first show how the cancellation occurs for the 6-particle potential $U^{h h}$, and then discuss $3 n$-particle potential $U^{h h \ldots h}$ for an arbitrary $n$. The terms corresponding to an arbitrary sequence of electron and hole decays can be analyzed analogously.

Consider connected terms of the cluster expansion containing $k$ functions $f^{h h}\left(\xi_{1}^{(1)}, \ldots, \xi_{6}^{(1)}\right), \ldots, f^{h h}\left(\xi_{1}^{(k)}, \ldots, \xi_{6}^{(k)}\right)$. To maximize the corresponding contribution one should connect them in such a way that one of the energy denominators coincides for all terms (two denominators cannot coincide, as it would require the coincidence of $\xi_{i}^{(j)}$ for all $j$ and each $i$, which is prohibited by the rules of the cluster expansion). Having fixed one of the denominators, one should keep the remaining free level positions independent of each other in order not to obtain smallness in $M$ or $T / \delta_{\zeta}$. As a result, two diagrams should be evaluated for each $k$, shown in Fig. C.1. Summing over $k$ and adding the leading term, we obtain

$$
\begin{aligned}
& \ln W_{h h}^{(2,0)}=-\sqrt{\pi s \Gamma^{(0,0)}}\left(\frac{2 \lambda M T}{\delta_{\zeta}}\right)^{2}\left[\ln \frac{\left(\Delta / \lambda \delta_{\zeta}\right)^{2}}{\sqrt{s \Gamma^{(0,0)}}}\right. \\
& \left.+\sum_{k=2}^{\infty} \frac{\left(-\Delta / \delta_{\zeta}\right)^{k-1}}{k !(k-1)} \int_{-\infty}^{\infty} \frac{\mathrm{d} x}{4} \frac{\cosh \frac{(k-1) x}{2}}{\cosh ^{k+1} \frac{x}{2}}+\sum_{k=2}^{\infty} \frac{\left(-\Delta / \delta_{\zeta}\right)^{k-1}}{k !(k-1)}\left(\frac{2 \lambda M T}{\delta_{\zeta}}\right)^{k-1}\right]
\end{aligned}
$$

where the logarithmic term in the square brackets is the leading one, corresponding to the fourth diagram of Fig. 14, the second and third terms corre- 
spond to the diagrams of Fig. C.1. The summation over $k$ is performed as

$$
\begin{aligned}
& \sum_{k=2}^{\infty} \frac{(-A)^{k-1}}{k !(k-1)}=-\int_{0}^{A} \mathrm{~d} x \sum_{k=2}^{\infty} \frac{(-x)^{k-2}}{k !}=-\int_{0}^{A} \frac{\mathrm{d} x}{x^{2}}\left(\mathrm{e}^{-x}-1+x\right) \\
& =-\int_{1 / A}^{\infty} \mathrm{d} x\left(\mathrm{e}^{-1 / x}-1+\frac{1}{x}\right) \approx-\ln A, \quad A \gg 1 .
\end{aligned}
$$

As a result, we obtain

$$
\ln W_{h h}^{(2,0)}=-\sqrt{\pi s \Gamma^{(0,0)}}\left(\frac{2 \lambda M T}{\delta_{\zeta}}\right)^{2} \ln \frac{M T /\left(\lambda^{2} \delta_{\zeta}\right)}{\sqrt{s \Gamma^{(0,0)}}} .
$$

This seems to correspond to the replacement

$$
\Delta \rightarrow \sqrt{M T \delta_{\zeta}}
$$

rather than to the promised Eq. (128).

To show that the transition point is nevertheless determined by Eq. (128), we analyze the hole channel for an arbitrary $n$. In this case the subleading logarithmic correction is given by diagrams where $n-1$ energy denominators are fixed by connections, and only one is independent of others. The independent denominator can be chosen in $n$ ways, of which $n-1$ produce diagrams analogous to the first diagram of Fig. C.1 (each $f$ has only one argument independent of other $f$ 's) and the last denominator produces a diagram similar to the second diagram of Fig. C.1 (each $f$ has three last arguments independent of other f's). The result is

$$
\begin{aligned}
& \ln W_{h \ldots h}^{(n, 0)}=-\sqrt{\pi s \Gamma^{(0,0)}}\left(\frac{2 \lambda M T}{\delta_{\zeta}}\right)^{n} \\
& \times\left[\frac{1}{(n-1) !} \ln ^{n-1} \frac{\left(\Delta / \lambda \delta_{\zeta}\right)^{n}}{\sqrt{s \Gamma^{(0,0)}}}-\frac{1}{(n-2) !} \ln ^{n-2} \frac{\left(\Delta / \lambda \delta_{\zeta}\right)^{n}}{\sqrt{s \Gamma^{(0,0)}}} \ln \left(\frac{M T}{\delta_{\zeta}} \frac{\Delta^{n}}{\delta_{\zeta}^{n}}\right)\right],
\end{aligned}
$$

The expression in the square brackets is nothing else but the first two terms of the binomial

$$
\begin{aligned}
& \frac{1}{(n-1) !}\left[\ln \frac{\left(\Delta / \lambda \delta_{\zeta}\right)^{n}}{\sqrt{s \Gamma^{(0,0)}}}-\ln \left(\frac{M T}{\delta_{\zeta}} \frac{\Delta^{n}}{\delta_{\zeta}^{n}}\right)\right]^{n-1} \\
& =\frac{1}{(n-1) !} \ln ^{n-1}\left(\frac{\delta_{\zeta}}{M T \lambda^{n}} \frac{1}{\sqrt{s \Gamma^{(0,0)}}}\right) .
\end{aligned}
$$

To obtain the next terms of the binomial one would have to connect the cluster functions in a way that fixes $n-2, n-3$, etc. denominators. As seen from 


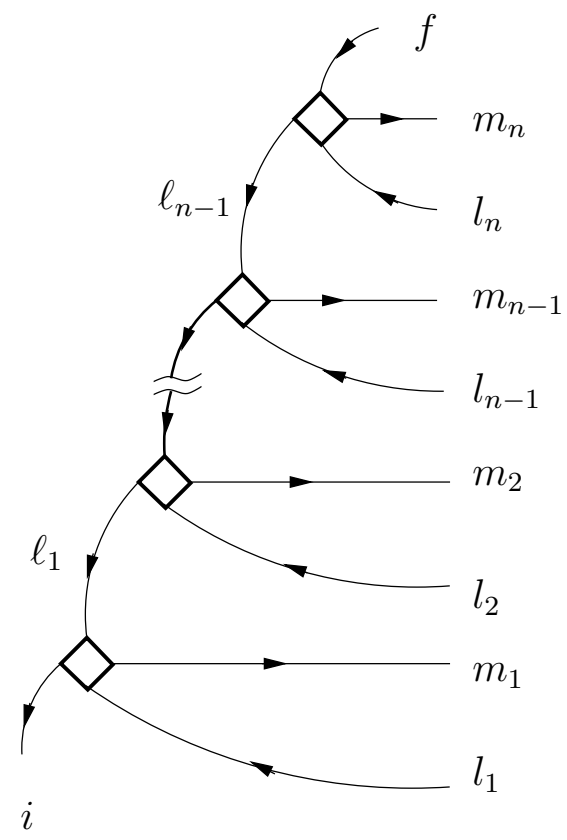

Fig. D.1. Amplitude of $n$th order of the peturbation theory used for the estimate of the distribution function (D.1). Being squared, these amplitudes generate the electron-like decay processes only (the first diagrams on Figs. 13, 14). External lines are assumed to be amputated and shown for the notation of orbitals and energy. Path $\ell$ in Eq. (149) corresponds to the sequence $\left\{\ell_{1}, \ell_{2}, \ldots \ell_{n-1}\right\}$ on this figure.

Eq. (C.6), the factor $M T / \delta_{\zeta}$ under the logarithm becomes unimportant at large $n$. This justifies the rule (128).

\section{Derivation of Eq. (151)}

Our goal in this Appendix is to evaluate the most dangerous contribtion from the interference corrections to the SCBA result. For this purpose, it suffices to demonstrate the calculation of $\alpha_{i n t}$ from Eq. (151) without numerical coeficient. Therefore, we will take into account only electron-like processes shown on Fig. D.1 and disregard other processes, cf. Figs. 13, 14. This leads to the underestimate of the overall numerical coefficient but does not affect the factorials.

The expression for such $n$th order amplitude in the insulating region reads

$$
\mathcal{A}_{i}^{f}\left(\left\{\ell_{k}\right\} ;\left\{l_{k}\right\} ;\left\{m_{k}\right\}\right) \simeq \frac{V_{i l_{1}}^{\ell_{1} m_{1}} F_{\ell_{1}, l_{1} ; m_{l}}^{\Rightarrow}}{\epsilon_{i}-\Xi_{l_{1} ; \ell_{1}}^{m_{1}}} \frac{V_{\ell_{1} l_{2}}^{\ell_{2} m_{2}} F_{\ell_{2}, l_{2} ; m_{2}}^{\Rightarrow}}{\epsilon_{i}-\Xi_{l_{1}, l_{2} ; \ell_{2}}^{m_{1}, m_{2}}} \cdots \frac{V_{\ell_{n-1} l_{n}}^{f m_{n}} F_{f, l_{n} ; m_{n}}^{\overrightarrow{m_{2}}}}{\epsilon_{i}-\Xi_{l_{1}, l_{2} \ldots, l_{n} ; l_{n}-1}^{m_{1}, m_{2}, m_{n}}}
$$

where the notation was introduced in Eqs. (105b) and (113). 
Expression (D.1) has the same form as those considered in Secs. 6.4 and 7.1. so that the machinery of Sec. 6.3 can be applied with small modification. First we fix the electron and hole levels $\xi_{l_{1}}, \ldots, \xi_{l_{n}} ; \xi_{m_{1}}, \ldots, \xi_{m_{n}}$ in Eq. (D.1), and sum over all the intermediate states $\ell_{1}, \ell_{2} ; \ldots, \ell_{n}$. Calculating the characteristic function, we perform the partial averaging - i.e., procedure of Eq. (102) with $2 n$ levels $l_{1}, \ldots, l_{n} ; m_{1}, \ldots, m_{n}$ excluded.

Using the linked cluster expansion and Eq. (48g), we find

$$
\begin{aligned}
& \ln \tilde{W}\left(q ;\left\{l_{i}\right\} ;\left\{m_{i}\right\}\right) \equiv \ln \left\langle\exp \left[i q \sum_{\ell_{k}} \mathcal{A}_{i}^{f}\left(\left\{\ell_{k}\right\} ;\left\{l_{k}\right\} ;\left\{m_{k}\right\}\right)\right]\right\rangle_{\left\{k_{i}\right\} ;\left\{m_{k}\right\}} \\
& \simeq-\pi|q| \delta_{\zeta} \lambda\left[2 \lambda \ln \frac{1}{\lambda}\right]^{n-1} \prod_{k=1}^{n} \frac{1-n_{l_{k}} n_{m_{k}}}{2}\left[\Upsilon^{2}\left(\Xi_{l_{1}}^{m_{1}}\right)-\Upsilon^{2}\left(\Xi_{l_{1}}^{i}\right)\right] \\
& \quad \times\left[\Upsilon^{2}\left(\Xi_{l_{2}}^{m_{2}}\right)-\Upsilon^{2}\left(\Xi_{l_{1} l_{2}}^{i m_{1}}\right)\right] \ldots\left[\Upsilon^{2}\left(\Xi_{l_{n}}^{m_{n}}\right)-\Upsilon^{2}\left(\Xi_{l_{1} l_{2} \ldots l_{n}}^{i m_{1} m_{2} \ldots m_{n-1}}\right)\right] .
\end{aligned}
$$

where we were dealing with the logarithmic integrals as in Sec. 7.3. For the sake of brievity we replaced $\Xi_{l_{1} \ldots}^{m_{1} \ldots} / \delta_{\zeta} \rightarrow \Xi_{l_{1} \ldots}^{m_{1} \ldots}$ in the last two lines of the equation.

Equation (D.2) enables us to estimate the distribution function of the amplitudes summed over the permutations among $n$ electrons and $n$ holes, see Eq. (149). Approximating the amplitudes obtained by the permutations of the final states to be independent of each other, cf. Fig. $20^{19}$ we find

$$
\begin{aligned}
\mathcal{W}\left(q ;\left\{l_{i}\right\} ;\left\{m_{i}\right\}\right) & \equiv\left\langle\exp \left[i q \sum_{\substack{\ell_{k} \\
P_{l} P_{m}}}(-1)^{P_{l}+P_{m}} \mathcal{A}_{i}^{f}\left(P_{l}\left\{l_{k}\right\} ; P_{m}\left\{m_{k}\right\}\right)\right]\right\rangle \\
& =\prod_{P_{l} P_{m}} \tilde{W}\left(q ; P_{l}\left\{l_{i}\right\} ; P_{m}\left\{m_{i}\right\}\right),
\end{aligned}
$$

where we used the standard expression for the characteristic function of sum, we suppressed the $\ell_{k}$ arguments of the amplitude for brevity, and the partial averaging as in Eq. (D.2) is implied.

\footnotetext{
$\overline{19}$ This corresponds to the leading logarithmic approximation in the linked cluster expansion. Note, however, that it does not affect the terms divergent as $n$ ! at all.
} 


$$
\begin{aligned}
\ln \left\langle\exp \left(-s \delta_{\zeta}^{-2} \sum_{\left\{l_{k}\right\},\left\{m_{k}\right\}} \gamma_{l}\left(\left\{l_{k}\right\},\left\{m_{k}\right\}\right)\right)\right\rangle \\
=\int \frac{\delta_{\zeta} \mathrm{d} q}{(2 \pi s)^{1 / 2}} \mathrm{e}^{-\frac{q^{2} \delta_{\zeta}^{2}}{2 s}} \prod_{k=1}^{2 n} \frac{\mathrm{d} \xi_{k}}{\delta_{\zeta}} \sum_{n_{k}= \pm 1} \frac{\exp \frac{\xi_{k} n_{k}}{2}}{2 \cosh \frac{\xi_{k} n_{k}}{2 T}} \\
\times \frac{\mathcal{W}(q ;\{1,2, \ldots n\} ;\{n+1, \ldots, 2 n\})-1}{(n !)^{2}} .
\end{aligned}
$$

Finally, expanding the last in Eq. (D.4) in powers of $|q|$, and performing remaining integrations, we obtain the structure of Eq. (151). Indeed, exponent contains $(n !)^{2}$ terms itself, so that the factorial factors cancel at all in the leading term. In the next term product $\mathcal{W}\left(q ;\left\{l_{k}\right\} ;\left\{m_{k}\right\}\right) \mathcal{W}\left(q ; P_{l}\left\{l_{k}\right\} ; P_{m}\left\{m_{k}\right\}\right)$ gives the same volume for the $\xi$ integration only for $P_{l}=P_{m}$ and impose the additional restricitions otherwise. The factor of $n$ ! is, thus, just a number of permutations electron-hole pairs without their destruction.

\section{References}

[1] P. W. Anderson, Phys. Rev. 109, 1492 (1958).

[2] D. J. Thouless, Phys. Rev. Lett. 39, 1167 (1977).

[3] E. Abrahams, P. W. Anderson, D. C. Licciardello, and T. V. Ramakrishnan Phys. Rev. Lett. 42, 673-676 (1979)

[4] M. E. Gertsenshtein and V. B. Vasil'ev, Teor. Veroyatn. Primen. 4, 424 (1959); ibid. 5, 3(E) (1959) [Theor. Probab. Appl. 4, 391 (1959); ibid 5, 340(E) (1959)].

[5] V.L. Berezinskii, Zh. Eksp. Teor. Fiz. 65, 1251 (1973) [Sov. Phys. JETP 38, 620 (1974)].

[6] K. B. Efetov and A. I. Larkin, Zh. Eksp. Teor. Fiz. 85, 764 (1983) [Sov. Phys. JETP 58, 444 (1983)].

[7] O. N. Dorokhov, Zh. Eksp. Teor. Fiz. 85, 1040 (1983) [Sov. Phys. JETP 58, 606 (1983)].

[8] N. F. Mott, J. Non-Crystal. Solids 1, 1 (1968).

[9] B. I. Shklovskii and A. L. Efros, Electronic Properties of Doped Semiconductors (Springer-Verlag, Berlin, 1984).

[10] L. Fleishman and P. W. Anderson, Phys. Rev. B 21, 2366 (1980).

[11] T. V. Shahbazyan and M. E. Raikh, Phys. Rev. B 53, 7299 (1996). 
[12] V. I. Kozub, S. D. Baranovskii and I. Shlimak, Solid State Commun. 113, 587 (2000).

[13] Z. Wang, M. P. A. Fisher, S. M. Girvin, and J. T. Chalker, Phys. Rev. B 61, 8326 (2000).

[14] T. Nattermann, T. Giamarchi, and P. Le Doussal, Phys. Rev. Lett. 91, 056603 (2003).

[15] B. L. Altshuler and A. G. Aronov, in Electron-Electron Interactions in Disordered Systems, edited by A. L. Efros and M. Pollak (North-Holland, Amsterdam, 1985).

[16] B. L. Altshuler, Y. Gefen, A. Kamenev, and L. S. Levitov, Phys. Rev. Lett. 78, 2803 (1997).

[17] R. Abou-Chacra, P. W. Anderson, and D. J. Thouless, J. Phys. C 6, 1734 (1973).

[18] I. L. Aleiner, B. L. Altshuler, and M. E. Gershenson, Waves in Random Media 9, 201 (1999).

[19] K. H. Fischer and J. A. Hertz, Spin glasses (Cambridge University Press, Cambridge, 1991); J.-P. Bouchaud, L. F. Cugliandolo, J. Kurchan and M. Mezard, in Spin Glasses and Random Fields ed. by A. P. Young (World Scientific, Singapore, 1998).

[20] A. D. Mirlin and Y. V. Fyodorov, Phys. Rev. B 56, 13393 (1997).

[21] A. A. Abrikosov, L. P. Gor'kov, and I. Ye. Dzyaloshinskii, Quantum Field Theoretical Methods in Statistical Physics (Pergamon Press, Oxford, 1965).

[22] K. B. Efetov, Zh. Eksp. Teor. Fiz. 92, 638 (1987); ibid. 93, 1125 (1987) [Sov. Phys. JETP 65, 360 (1987); ibid. 66, 634 (1987)].

[23] The properties of the matrix elements in the metallic regime can be extracted from the results of Ref. [15], see Sec. 2.3 of the review by I.L. Aleiner, P.W. Brouwer, and L.I. Glazman, Phys. Rep. 358, 309 (2002). More precise derivation can be found in the review by A. D. Mirlin, Phys. Rep. 326, 259 (2000).

[24] L. V. Keldysh, Zh. Éksp. Teor. Fiz. 47, 1945 (1964) [Sov. Phys. JETP 20, 1018 (1977)]; we use the parametrization of A. I. Larkin and Y. N. Ovhcninnikov, Zh. Éksp. Teor. Fiz. 73, 299 (1977) [Sov. Phys. JETP 46, 155 (1977)].

[25] A. A. Gogolin, V. I. Melnikov, and É. I. Rashba, Zh. Eksp. Teor. Fiz. 69, 327 (1975) [Sov. Phys. JETP 42, 168 (1975)].

[26] M. E. Raikh and I. M. Ruzin, in "Mesoscopic Phenomena in Solids", ed. by B. L. Altshuler, P. A. Lee, and R. A. Webb (North-Holland, Amsterdam, 1991).

[27] A. M. Dykhne, Zh. Eksp. Teor. Fiz. 59, 63 (1970) [Sov. Phys. JETP 32, 110 (1971)].

[28] I. V. Gornyi, A. D. Mirlin, and D. G. Polyakov, cond-mat/0407305, version 1. 
[29] G. E. Mayer and M. Göppert-Mayer, Statistical Mechanics (Wiley, New York, 1948).

[30] See G. F. Lawler, Intersections of Random Walks (Birkhäuser, Boston, 1996).

[31] G. M. Eliashberg, Zh. Eksp. Teor. Fiz. 42, 1658 (1962) [Sov. Phys. JETP 15, 1151 (1962)].

[32] S. I. Khondaker, I. S. Shlimak, J. T. Nicholls, M. Pepper, and D. A. Ritchie, Phys. Rev. B 59, 4580 (1999).

[33] S. Marnieros, L. Bergé, A. Juillard, and L. Dumoulin, Phys. Rev. Lett. 84, 2469 (2000).

[34] M. E. Gershenson, Yu. B. Khavin, D. Reuter, P. Schafmeister, and A. D. Wieck, Phys. Rev. Lett. 85, 1718 (2000).

[35] A. Ghosh, M. Pepper, D. A. Ritchie, E. H. Linfield, R. H. Harrell, H. E. Beere, and G. A. C. Jones, phys. stat. sol. (b) 230, 211 (2002).

[36] A. I. Yakimov, A. V. Dvurechenskii, A. I. Nikiforov, and A. A. Bloshkin, Pis'ma Zh. Éksp. Teor. Fiz. 77, 445 (2003) [JETP Letters 77, 376 (2003)].

[37] N. F. Mott, Proc. Phys. Soc. London, Sect A 62, 416 (1949).

[38] J. Hubbard, Proc. R. Soc. London, Ser. A 281, 401 (1964).

[39] E. P. Wigner, Phys. Rev. 46, 1002 (1934).

[40] A. I. Larkin, Zh. Eksp. Teor. Fiz. 58, 1466 (1970) [Sov. Phys. JETP 31, 784 (1971)].

[41] I. V. Gornyi, A. D. Mirlin, and D. G. Polyakov, cond-mat/0506411. 